UNIVERSIDADE DE SÃO PAULO

Instituto de Astronomia, Geofísica e Ciências Atmosféricas

Departamento de Astronomia

\author{
Raniere Maciel de Menezes
}

\title{
Variabilidade de AGNs em raios gama no Universo local
}




\section{Raniere Maciel de Menezes}

\section{Variabilidade de AGNs em raios gama no Universo local}

Dissertação apresentada ao Departamento de Astronomia do Instituto de Astronomia, Geofísica e Ciências Atmosféricas da Universidade de São Paulo como requisito parcial para obtenção do título de Mestre em Astronomia.

Área de concentração: Astronomia. Orientador: Rodrigo Nemmen.

Versão corrigida. O original encontra-se disponível na Unidade. 
À memória dos dias que virão. 
"Mas vamos tratar da chegada Quando Lampião bateu Um diabo ainda moço No portão apareceu: - Quem é você, cavalheiro? - Moleque, sou cangaceiro! Lampião lhe respondeu

- Moleque, não! Sou vigia E não sou seu pariceiro E hoje aqui o senhor não entra Sem dizer quem é primeiro - Moleque, abra o portão Saiba que sou Lampião O assombro do mundo inteiro!"

A chegada de Lampião no inferno José Pacheco da Rocha 


\section{Agradecimentos}

"Um homem não pode esmorecer", dizia meu avô, diz meu pai. Talvez por isso eu tenha chegado até aqui. Não sei. Sei que a primeira palavra que falei foi mãe. Não lembro. Sei. Estava com fome, acho. Falei chorando. Depois eles me deram um irmão. Sem que eu pedisse mesmo. Acho que me deram só um para que eu aprendesse a valorizá-lo. Funcionou. Obrigado.

Não fosse por todo o apoio do meu orientador, Rodrigo Nemmen, este projeto dificilmente teria sido desenvolvido. O agradeço não só por sua dedicação ao nosso trabalho, mas principalmente pela confiança que depositou em mim. Ainda agradeço de coração a todos os meus amigos e companheiros de trabalho, que me deram todo o suporte necessário para enfrentar as caatingas da Universidade/cidade de São Paulo. Agradeço especialmente ao César Costa, ao Carlos Eduardo Lopes e à colaboração Fermi-LAT por todo o conhecimento que me passaram e por toda paciência que tiveram comigo.

Por fim, agradeço à CAPES e à FAPESP pelo auxílio financeiro, sem o qual este trabalho não seria possível. 


\section{Resumo}

Desde seu lançamento em 2008, o Fermi Gamma-ray Space Telescope abriu uma nova janela para o lado extremo do Universo e vem explorando aceleradores de partículas astrofísicos como buracos negros supermaciços em núcleos ativos de galáxias (AGNs), explosões de estrelas no Universo longínquo e vários outros fenômenos. Neste projeto, investigamos um conjunto de 15 AGNs de baixa luminosidade do Palomar survey nas proximidades da Via Láctea com base em mais de sete anos de dados do Large Area Telescope, a bordo do Fermi. Caracterizamos a variabilidade de suas curvas de luz em raios $\gamma$, restringindo, assim, a natureza de seus processos de altas energias (basicamente usando a escala de tempo de variabilidade para restringir o tamanho da região emissora de raios $\gamma$ ). Entretanto, descobrimos que a maior parte da nossa amostra não emite fótons o suficiente para ser detectada pelo Fermi-LAT.

O curioso comportamento periódico de algumas fontes também foi explorado. Aplicamos técnicas de análise de séries temporais, como transformada de Fourier, periodograma de Lomb-Scargle e continuous wavelet transform, que nos trouxe algumas conclusões interessantes juntamente com algumas descobertas fortuitas. Em particular, encontramos evidência de QPO na NGC 1275, com um período de aproximadamente 570 dias, e um comportamento periódico não-estacionário no BL Lac PG 1553+113. 


\section{Abstract}

Since its launch in 2008, the Fermi Gamma-ray Space Telescope opened a new window into the extreme side of the universe and has been observing $\gamma$-rays from astrophysical particle accelerators such as supermassive black holes in active galactic nuclei (AGN), exploding stars in the distant universe and many others phenomena. In this project, we investigate a sample of 15 nearby low-luminosity AGNs from Palomar survey based on Fermi Large Area Telescope (LAT) data. We characterize the $\gamma$-ray variability of these sources in order to constrain the nature of its high-energy processes (basically using the variability timescales to constrain the size of the $\gamma$-ray emission region). However, most of our sample does not emit enough photons to be detected by Fermi-LAT.

The odd periodic behavior in the light curve of some sources was also explored. We applied time-series analysis techniques, such as fast Fourier transform, Lomb-Scargle periodogram and continuous wavelet transform, which gave us some interesting conclusions besides some serendipitous discoveries. In particular we found evidence of QPO in NGC 1275 with a period around 570 days and a non-stationary periodic behavior in PG $1553+113$. 
\begin{tabular}{lll}
\hline O céu em raios gama & 1
\end{tabular}

1.1 Fermi-LAT . . . . . . . . . . . . . . . . . . . . . . . . . 2

1.1 .1 Tracker . . . . . . . . . . . . . . . . . 3

1.1 .2 Calorímetro . . . . . . . . . . . . . . . . . . . . . . . . 3

1.1.3 Anticoincidence Detector . . . . . . . . . . . . . . . . . . 5

1.1.4 Data Acquisition System . . . . . . . . . . . . . . . . . . . . 5

1.1.5 Point spread function . . . . . . . . . . . . . . . . 5

1.2 Telescópios Cherenkov . . . . . . . . . . . . . . . . . . 6

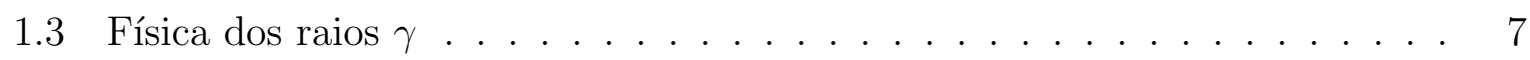

1.3 .1 Aberração relativística . . . . . . . . . . . . . . . . . . 8

1.3 .2 Efeito Doppler . . . . . . . . . . . . . . . . . . . . . . . . . 9 9

1.3 .3 Síncrotron . . . . . . . . . . . . . . . . . . . . . . . . . . 10

1.3 .4 Espalhamento Compton inverso . . . . . . . . . . . . . . . . . 14

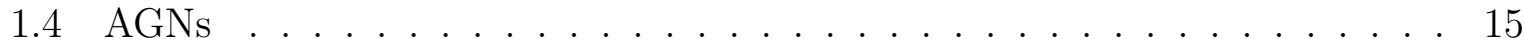

1.4 .1 LINERS . . . . . . . . . . . . . . . . . . . . . . 16

1.4 .2 Galáxias Seyfert . . . . . . . . . . . . . . . . . . . . . 18

1.4 .3 Rádio-galáxias . . . . . . . . . . . . . . . . . . . . . . . . . . . . 18

1.4.4 Blazares . . . . . . . . . . . . . . . . . . . . . . . . . . . 19

1.4 .5 Buracos negros e discos de acreção . . . . . . . . . . . . . . 23

1.4 .6 Modelo unificado . . . . . . . . . . . . . . . . . . . . . . 28

1.5 Emissão difusa $\ldots \ldots \ldots$. . . . . . . . . . . . . . . . . . . . . . . . . . . . . 29

1.6 Horizonte cósmico de raios $\gamma \ldots \ldots \ldots \ldots$. . . . . . . . . . . . . 31

1.7 Motivação e objetivos . . . . . . . . . . . . . . . . . . . . . . . . . 32

\begin{tabular}{|lll}
\hline 2 & Explorando os dados do Fermi & 34
\end{tabular}

2.1 Metodologia . . . . . . . . . . . . . . . . . . . . . . . . . . 35 
2.1 .1 Maximum likelihood estimation . . . . . . . . . . . . . . 36

2.1 .2 Mapas de teste estatístico . . . . . . . . . . . . . . . 38

$2.2 \quad$ Pipeline de produção de curvas de luz . . . . . . . . . . . . . . . 38

2.2 .1 Testes da pipeline $\ldots \ldots \ldots \ldots$

2.3 Análise de séries temporais . . . . . . . . . . . . . . . . . . . . 41

$2.3 .1 \quad$ Fourier e Lomb-Scargle . . . . . . . . . . . . . . . . . . . . . 42

2.3 .2 Continuous wavelet transform . . . . . . . . . . . . . 44

$\begin{array}{lll}3 & \text { Resultados } & 50\end{array}$

3.1 Amostra . . . . . . . . . . . . . . . . . . 50

3.2 Curvas de luz e mapas de TS . . . . . . . . . . . . . . . . . 52

3.2 .1 Perseus A . . . . . . . . . . . . . . . . . . . . 52

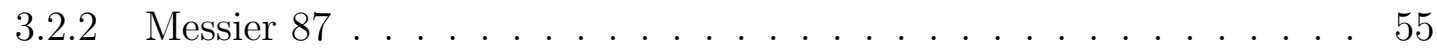

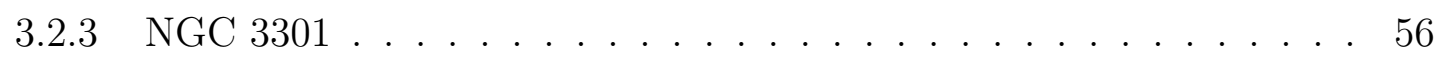

$3.2 .4 \quad$ NGCs 4762,3627 e $3623 \ldots \ldots \ldots \ldots \ldots$

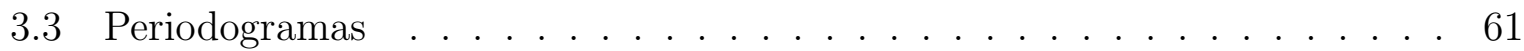

$3.3 .1 \quad$ PG $1553+113 \ldots \ldots \ldots \ldots \ldots \ldots$

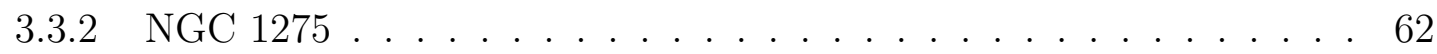

3.4 Evidência de novas fontes de raios $\gamma \ldots \ldots \ldots \ldots \ldots$

$\begin{array}{|ll|}4 \text { Conclusões e perspectivas } & 76\end{array}$ 


\section{Lista de abreviaturas}

ACD Anticoincidence Detector

ADAF Escoamento de acreção dominado por advecção (Advection-Dominated Accretion Flow)

AGN Núcleo ativo de galáxia (Active Galactic Nuclei)

BLR Região de linhas largas (Broad Line Region)

CGRH Horizonte cósmico de raios $\gamma$ (Cosmic Gamma Ray Horizon)

CWT Continuous Wavelet Transform

DAQ Data Acquisition System

EBL Luz de fundo extragaláctica (Extragalactic Background Light)

EGB Fundo extragaláctico de raios $\gamma$ (Extragalactic Gamma-ray Background)

FFT Transformada rápida de Fourier (Fast Fourier Transform)

FR-I Fanaroff-Riley I

FR-II Fanaroff-Riley II

FSRQ Flat Spectrum Radio Quasar

GBM Gamma-ray Burst Monitor

LAT Large Area Telescope

LINER Low-ionization Nuclear Emision-line Region

LLAGN Núcleo ativo de galáxia de baixa luminosidade (Low-luminosity Active Galactic Nuclei)

LMC Grande Nuvem de Magalhães (Large Magellanic Cloud)

LSP Periodograma de Lomb-Scargle (Lomb-Scargle Periodogram)

MLE Estimativa de máxima verossimilhança (Maximum Likelihood Estimation)

NLR Região de linhas estreitas (Narrow Line Region)

PSF Point Spread Function

QPO Objeto quasi-periódico (Quasi-periodic object) 
ROI Região de interesse (Region of Interest)

SED Distribuição espectral de energia (Spectral Energy Distribution)

SMBH Buraco negro supermaciço (Super Massive Black Hole)

SMC Pequena Nuvem de Magalhães (Small Magellanic Cloud)

SSC Synchrotron Self-Compton

TS Teste estatístico (Test Statistic)

VLBI Very-long-baseline interferometry 


\section{CAPÍTULO 1}

\section{O céu em raios gama}

Raios $\gamma$, a forma mais energética de radiação eletromagnética, são oriundos de fontes não-térmicas e são uma peça fundamental no estudo de fenômenos de alta energia, tais como jatos emitidos por núcleos ativos de galáxias (active galactic nuclei - AGN, daqui em diante), explosões estelares, pulsares e, potencialmente, fenômenos mais exóticos como aniquilação de matéria escura (Ackermann et al., 2015c) e gravitação quântica.

A detecção direta de raios $\gamma$, entretanto, deve ser feita por telescópios espaciais, já que a atmosfera terrestre é opaca à radiação de alta energia (raios $\gamma$, raios $\mathrm{X}$ e boa parte do ultra violeta). Lançado em 2008, o Fermi Gamma-ray Space Telescope vem mapeando o céu diariamente e contribuindo de maneira fundamental na astrofísica de altas energias.

O instrumento primário do Fermi, o Large Area Telescope (LAT), possui área efetiva, resolução espacial e temporal, campo de visão e intervalo de energias muito maiores que as de seu antecessor, o Compton Gamma Ray Observatory (CGRO). Enquanto o CGRO levou um ano e meio para mapear todo o céu, o Fermi-LAT produz um mapa completo do céu a cada $\sim 3$ horas.

O outro instrumento abordo é o Gamma-ray Burst Monitor (GBM), que possui um campo de visão muito maior que o LAT e faz a cobertura temporal e espectral de fenômenos transientes como gamma-ray bursts entre $10 \mathrm{keV}$ e $40 \mathrm{MeV}$. Para as análises feitas neste projeto, no entanto, o LAT foi o único instrumento utilizado.

Iniciaremos este capítulo com uma introdução ao telescópio Fermi, onde exploraremos os principais componentes do LAT e deixaremos clara a sua maneira de obtenção de dados. Seremos breves com uma introdução aos telescópios Cherenkov e trataremos, a seguir, da física da produção de raios $\gamma$ e da fenomenologia de vários tipos de AGNs, no intuito de criar um alicerce para a melhor compreensão deste trabalho. Uma vez com a base estabelecida, falaremos dos motivos que nos levaram a realizar este projeto. 


\section{$1.1 \quad$ Fermi-LAT}

Similar a detectores usados em aceleradores de partículas, o LAT é uma matriz de $4 \times 4$ torres idênticas (figura 1.1), cada uma com um tracker de placas de silício, onde os raios $\gamma$ interagem convertendo-se em pares de elétron-pósitron, e um calorímetro, onde a energia dos pares formados (e então do fóton) é medida (figura 1.2). O instrumento é coberto por um cintilador plástico, o Anticoincidence Detector (ACD), para filtrar a enorme quantidade de raios cósmicos encontrados na órbita baixa da Terra, na qual o telescópio se encontra.

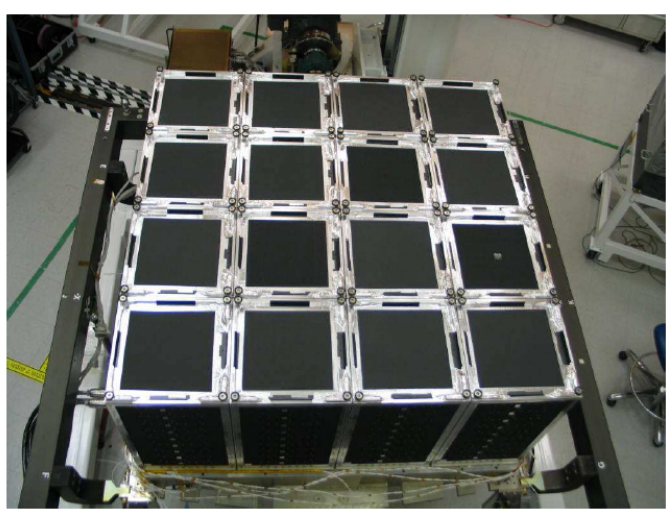

Figura 1.1: matriz de placas de silício antes de ser acoplado ao ACD. Fonte: Atwood et al. (2009).

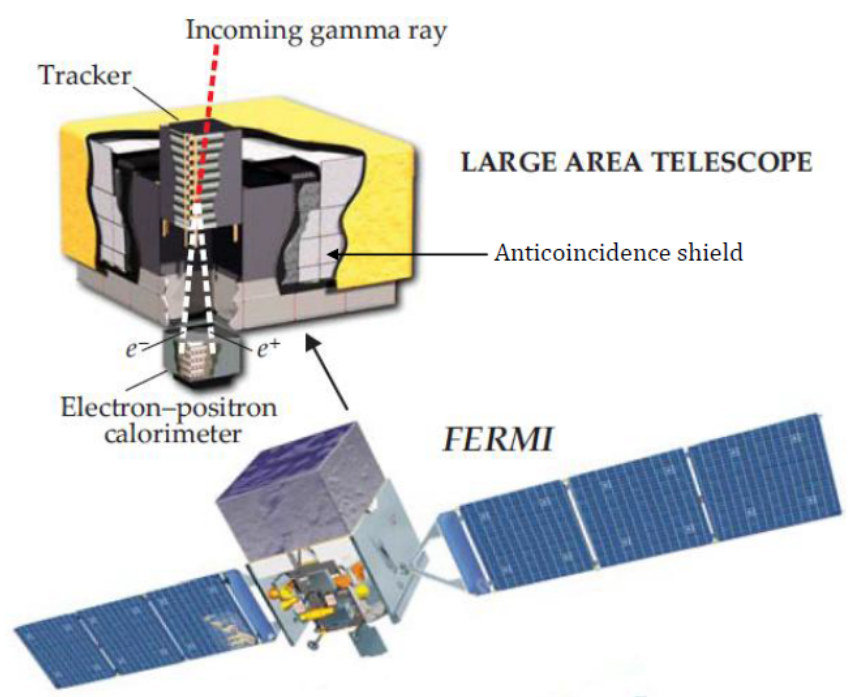

Figura 1.2: telescópio Fermi e LAT, com seu "tracker", calorímetro e anticoincidence detector. Fonte: Thompson et al. (2012), imagem editada.

A sensibilidade do LAT, que opera entre $20 \mathrm{MeV}$ e $\sim 500 \mathrm{GeV}$, é maior em torno de $1 \mathrm{GeV}$, onde a área efetiva do telescópio é aproximadamente $0,8 \mathrm{~m}^{2}$ (figura 1.3), seu campo de visão é em torno de 2,4 sr e sua resolução angular (dentro de $1 \sigma$ ), considerando a detecção de um único fóton, é melhor que $1^{o}$ (Massaro et al., 2016). 
O cérebro por trás do Fermi é o Data Acquisition System (DAQ), que coleta informações do tracker, do calorímetro e do Anticoincidence Detector e faz a distinção inicial entre sinais indesejados causados por raios cósmicos e sinais causados por raios $\gamma$.

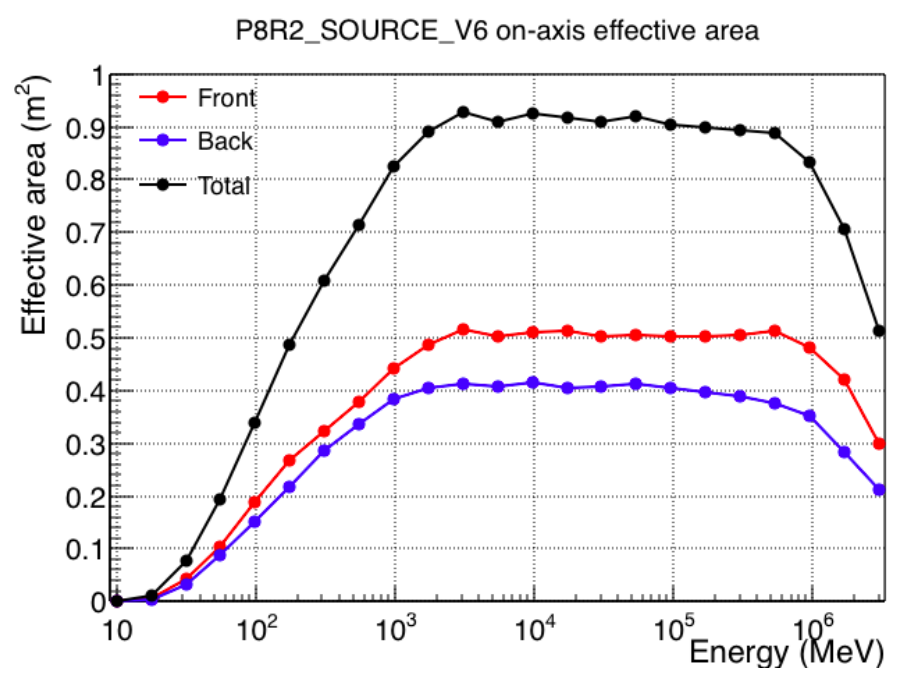

Figura 1.3: área efetiva do telescópio como função da energia para fótons de incidência paralela ao eixo polar do telescópio (linha preta). Fonte: Wood (2016).

\subsubsection{Tracker}

Raios $\gamma$ não podem ser refletidos ou refratados. Sua direção de chegada no telescópio é medida de forma indireta, através da conversão do fóton em um par elétron-pósitron no tracker.

Cada uma das 16 torres do tracker possui várias camadas de folhas finas de tungstênio, onde os fótons são convertidos em pares $e^{+} e^{-}$. Estas camadas são intercaladas com detectores de silício que registram a passagem de partículas carregadas, medindo, então, o caminho das partículas resultantes da conversão de pares. Esta informação é usada para reconstruir a direção do raio $\gamma$ incidente (figura 1.4).

\subsubsection{Calorímetro}

Os propósitos principais do calorímetro são (i) medir a energia da cascata de partículas resultante do par $e^{-} e^{+}$produzido pelo fóton $\gamma$ incidente e (ii) mapear o perfil da cascata, providenciando um importante discriminador de background.

Cada módulo do calorímetro possui 96 cristais de $\mathrm{CsI}(\mathrm{Tl}$ ) (iodeto de césio dopado com tálio), um cintilador que produz flashes de luz cuja intensidade é proporcional à energia das partículas incidentes; é comumente utilizado em física de altas energias. Os cristais são isolados opticamente uns dos outros e arranjados em oito camadas, como mostrado na figura 1.5 . 


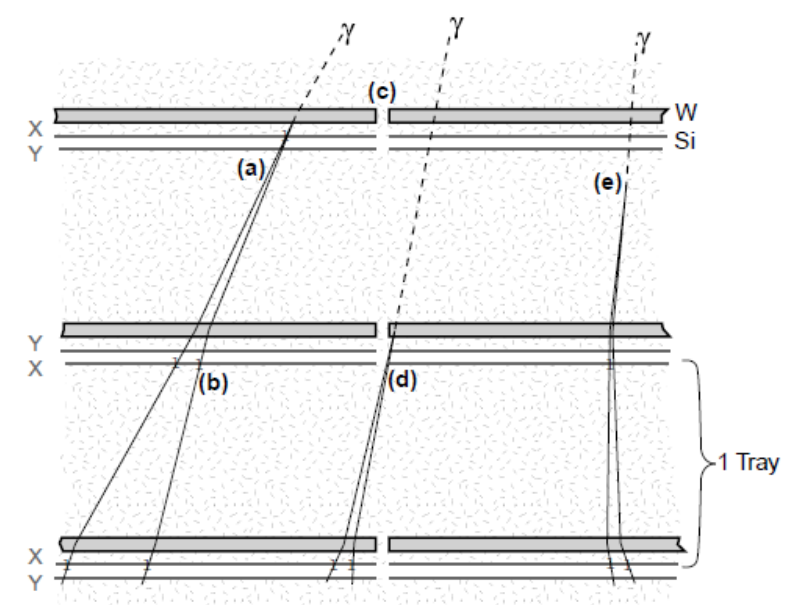

Figura 1.4: ilustração do funcionamento do tracker. (a) Conversão ideal na folha de tungstênio W: os detectores de silício são localizados o mais próximo possível destas folhas, na tentativa de detectar as partículas com o mínimo de espalhamento. O espalhamento na segunda folha de tungstênio causa muito pouco impacto na medida. (b) O par $e^{-} e^{+}$ pode ser detectado em várias camadas, melhorando a point spread function (PSF) e a rejeição de raios cósmicos. (c) As folhas conversoras cobrem apenas a área ativa do silício no intuito de minimizar conversões nas quais uma medida com pouco espalhamento não é possível. (d) Uma interação perdida na primeira ou segunda camada pode degradar a PSF por até um fator de dois. (e) Conversões podem ocorrer no matérial que compõe a estrutura do telescópio ou nas placas de silício, fazendo com que os pares produzidos sejam bastante espalhados antes de serem medidos. Para reduzir este problema, o material entre as camadas é o mínimo possível. Fonte: Atwood et al. (2009).

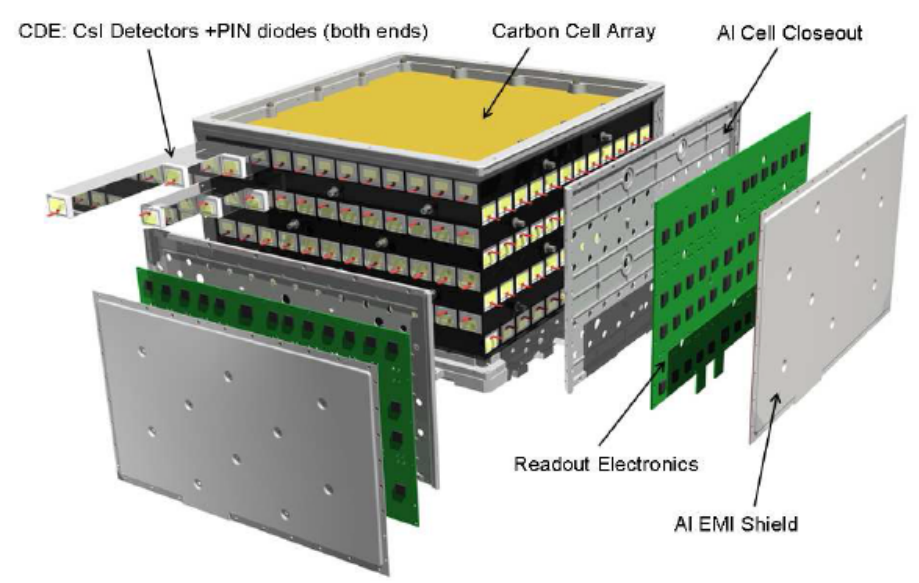

Figura 1.5: esquema do módulo do calorímetro no LAT. Cada elemento do cristal possui dois fotodiodos, um em cada extremidade, que medem a luz gerada pela cintilação das partículas da cascata. Fonte: Atwood et al. (2009). 
De acordo com o perfil longitudinal da cascata, uma estimativa da energia inicial do par $e^{-} e^{+}$é derivada ajustando-se os dados com uma descrição analítica do perfil longitudinal esperado. A resolução em energia resultante é limitada por flutuações na cascata (Atwood et al., 2009).

\subsubsection{Anticoincidence Detector}

A principal função do ACD é rejeitar o background de raios cósmicos. Sua eficiência é de 99,97\% para a detecção de uma única partícula carregada entrando no campo de visão do LAT (Atwood et al., 2009).

O ACD é composto por blocos plásticos que cintilam na presença de raios cósmicos. A luz gerada pela cintilação em cada bloco é coletada por fibras óticas e o sinal é, então, rejeitado. A figura 1.2 mostra o ACD integrado ao tracker.

\subsubsection{Data Acquisition System}

Sendo responsável por coletar os dados dos outros três subsistemas, o DAQ providencia, ainda a bordo da espaçonave, o processamento dos algoritmos de filtragem de raios cósmicos, para reduzir o número de eventos a serem enviados à Terra (reduzem a taxa de eventos de $2-4 \mathrm{kHz}$ para $\sim 400 \mathrm{~Hz}$ ), e executa uma plataforma de análise científica que detecta rapidamente fenômenos transientes.

O tempo morto instrumental por evento detectado é $\geq 26,50 \mu \mathrm{s}$ (Atwood et al., 2009), que é o tempo mínimo requerido para a leitura do evento pelo DAQ. Qualquer evento que ocorra menos de 26,50 $\mu$ s após um primeiro evento, será ignorado pelo LAT.

\subsubsection{Point spread function}

A resolução angular do LAT depende da energia dos raios $\gamma$, de seu ângulo de incidência, do local onde os fótons interagem no detector e da quantidade de fótons que chegam da fonte observada. Quanto maior a energia e o número de fótons detectados, maior é a resolução angular (menor point spread function - PSF). A figura 1.6 mostra imagens que obtivemos do blazar 3C 279. As únicas diferenças entre as imagens são o intervalo de energia trabalhado e o número de fótons incidentes. Notamos claramente a diminuição na PSF para altas energias.

Uma das tarefas mais complexas do LAT foi encontrar o equilíbrio entre a necessidade por conversores finos, para obtenção de boa PSF em baixas energias, onde a PSF é determinada primariamente por uma dependência de $\sim 1 / E$, versus a necessidade de maximização da área efetiva, importante em altas energias (Atwood et al., 2009). A solução foi dividir o tracker em duas regiões, "frente" e "costas". A região frontal (doze primeiras camadas) possuem conversores finos, cada um com 0,03 comprimentos de radiação 

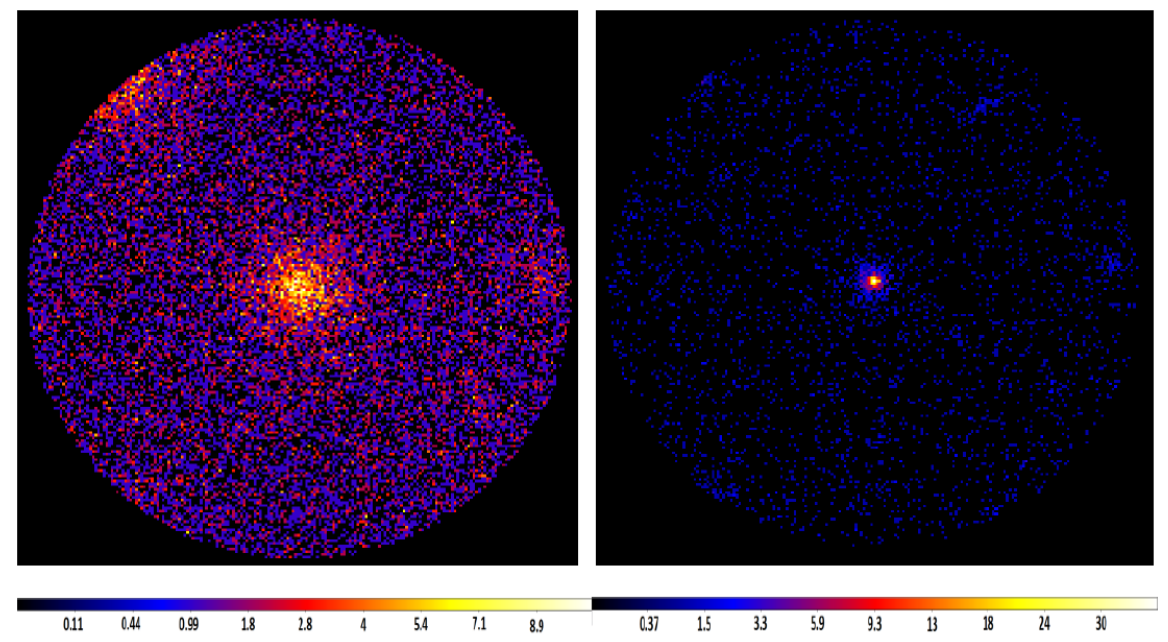

Figura 1.6: blazar 3C 279. A imagem da esquerda foi obtida num intervalo de energia entre $100 \mathrm{MeV}$ e $500 \mathrm{MeV}$, onde notamos uma alta PSF. À direita, o mesmo blazar, agora analisado entre $1 \mathrm{GeV}$ e $100 \mathrm{GeV}$. A barra representa a contagem de fótons. O período de exposição foi de seis meses.

de espessura, para otimizar a PSF em baixas energias, enquanto que as últimas quatro camadas conversoras são $\sim 6$ vezes mais espessas, para maximizar a área efetiva, com uma perda de menos de um fator dois em resolução angular (em torno de $1 \mathrm{GeV}$ ) para fótons convertidos nesta região (Atwood et al., 2009).

\subsection{Telescópios Cherenkov}

Quando um raio $\gamma$ de alta energia (acima de algumas dezenas de $\mathrm{GeV}$ ) entra na atmosfera, este interage com um nucleo atômico e gera uma cascata de partículas, que movem-se pela atmosfera com velocidade superior à da luz no meio e emitem, por consequência do efeito Cherenkov, radiação principalmente no azul e ultravioleta. Para cascatas aproximadamente verticais, a radiação de Cherenkov ilumina um círculo com diâmetro de cerca de $250 \mathrm{~m}$ no chão (Actis et al., 2010). Para maiores ângulos de zênite, esta área pode crescer consideravelmente. A radiação Cherenkov pode ser observada por instrumentos ópticos e ser usada para imagear a cascata (figura 1.7). Reconstruindo espacialmente o eixo da cascata, podemos determinar a direção de chegada do fóton incidente. Estas cascatas eletromagnéticas são, no entanto, muito fracas se comparadas à emissão de fundo do céu e, portanto, grandes refletores ópticos, operando apenas em noites muito escuras, com área coletora acima de $100 \mathrm{~m}^{2}$, são necessários para detectá-las (Actis et al. 2010).

Em telescópios Cherenkov, a área efetiva do detector possui aproximadamente o tamanho do Cherenkov pool ( $250 \mathrm{~m}$ - figura 1.7) no nível do solo (Actis et al., 2010), o que é cerca de $10^{5}$ vezes maior do que a área efetiva dos detectores em satélites. Entretanto, o fluxo de fótons decresce consideravelmente com o aumento da energia. Diferentemente do telescópio Fermi, os telescópios Cherenkov não possuem um campo de visão tão amplo 


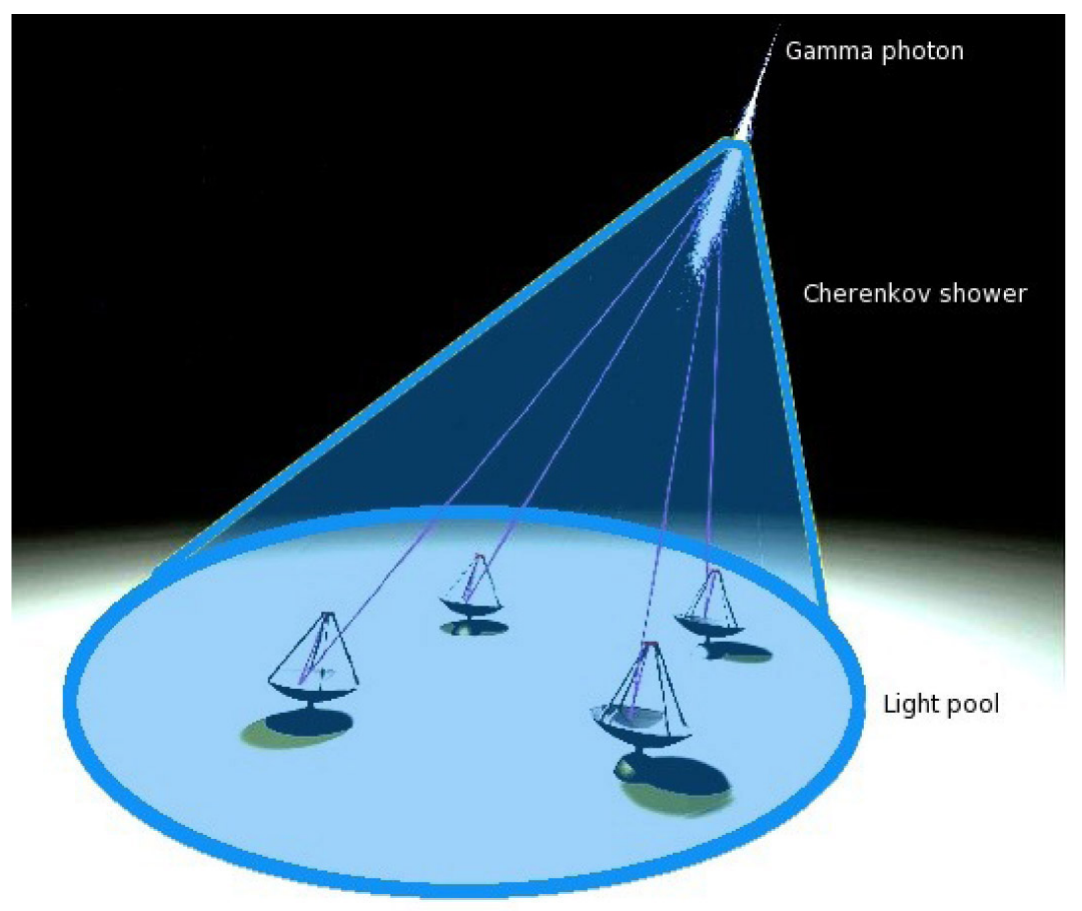

Figura 1.7: visão esquemática do funcionamento de telescópios Cherenkov. Fonte: Rodolfo Canestrari. Retirada de http://spie.org/newsroom/ 3727-reflecting-surfaces-of-novel-cherenkov-telescopes.

e não podem criar um all-sky survey com a mesma facilidade.

Se tratando de raios $\gamma$ de energia muito alta (VHE - energias $>100 \mathrm{GeV}$ ), os instrumentos H.E.S.S 1 MAGIC 22 e VERITAS 3 vêm apresentando resultados importantes nos últimos anos $4^{5} 5^{6}$. Resultados estes que deverão ser melhorados com a chegada do Cherenkov Telescope Array (CTA), que contará com tecnologia muito superior a seus antecessores (Actis et al., 2010).

\subsection{Física dos raios $\gamma$}

O processo de produção de raios $\gamma$ nas fontes que vamos considerar se deve principalmente aos efeitos síncrotron e synchrotron self-Compton (SSC), que dependem de fortes campos magnéticos e partículas com velocidades relativísticas. Efeitos secundários, como o bremsstrahlung e o decaimento de píons, que são os responsáveis pela maior parte da emissão em $\gamma$ da Via Láctea e de galáxias comuns, não serão tratados aqui.

Apesar de não produzir raios $\gamma$, a compreensão do efeito de beaming é essencial para o

\footnotetext{
1 https://www.mpi-hd.mpg.de/hfm/HESS/. Acesso em 05/10/2016.

2 https://magic.mpp.mpg.de/. Acesso em 05/10/2016.

3 http://veritas.sao.arizona.edu/ Acesso em 05/10/2016.

${ }^{4}$ https://www.mpi-hd.mpg.de/hfm/HESS/pages/publications/. Acesso em 05/10/2016.

5 http://veritas.sao.arizona.edu/veritas-science/veritas-results-mainmenu-72. Acesso
} em $05 / 10 / 2016$.

6 https://magic.mpp.mpg.de/backend/publications/articles. Acesso em 05/10/2016. 
melhor entendimento dos efeitos síncrotron e SSC, sendo este o responsável pela colimação da radiação emitida por uma partícula na direção e sentido do seu vetor velocidade.

\subsubsection{Aberração relativística}

Dadas as transformações de Lorentz para dois referenciais inerciais, $K$ e $K^{\prime}$, movendose com velocidade $v$, um em relação ao outro, na direção do eixo $x$ :

$$
\begin{gathered}
d x=\gamma\left(d x^{\prime}+v d t^{\prime}\right) \\
d y=d y^{\prime} \\
d z=d z^{\prime} \\
d t=\gamma\left(d t^{\prime}+\frac{v}{c^{2}} d x^{\prime}\right),
\end{gathered}
$$

uma partícula com velocidade $u^{\prime}$ em $K^{\prime}$ terá velocidade dada por

$$
u_{x}=\frac{d x}{d t}=\frac{\gamma\left(d x^{\prime}+v d t^{\prime}\right)}{\gamma\left(d t^{\prime}+\frac{v}{c^{2}} d x^{\prime}\right)}=\frac{u_{x}^{\prime}+v}{1+\frac{v u_{x}^{\prime}}{c^{2}}}
$$

e, analogamente,

$$
u_{y}=\frac{u_{y}^{\prime}}{\gamma\left(1+\frac{v u_{x}^{\prime}}{c^{2}}\right)} \quad \& \quad u_{z}=\frac{u_{z}^{\prime}}{\gamma\left(1+\frac{v u_{x}^{\prime}}{c^{2}}\right)}
$$

no referencial $K$. Se em $K^{\prime}$ a partícula move-se na direção $\theta^{\prime}$ dada por $\tan \theta^{\prime}=u_{y}^{\prime} / u_{x}^{\prime}$ (considerando apenas movimento no plano x-y), em $K$, a direção será

$$
\tan \theta=\frac{u_{y}}{u_{x}}=\frac{u_{y}^{\prime}}{\gamma\left(1+\frac{v u_{x}^{\prime}}{c^{2}}\right)} \frac{\left(1+\frac{v u_{x}^{\prime}}{c^{2}}\right)}{u_{x}+v}=\frac{u_{y}^{\prime}}{\gamma\left(u_{x}^{\prime}+v\right)},
$$

mas como $u_{y}^{\prime}=u^{\prime} \sin \theta^{\prime}$ e $u_{x}^{\prime}=u^{\prime} \cos \theta^{\prime}$, temos:

$$
\tan \theta=\frac{u^{\prime} \sin \theta^{\prime}}{\gamma\left(u^{\prime} \cos \theta^{\prime}+v\right)}
$$

Da equação acima é fácil notar que, se uma partícula move-se perpendicularmente a $v$ em $K^{\prime}$ (ou seja, se $\theta^{\prime}=\pi / 2$ ), o mesmo não ocorre no referencial $K$ ! Em $K$, a partícula tem duas componentes de velocidade: $u_{x}=v$ e $u_{y}=u^{\prime} / \gamma$ (ver equações $1.2 \mathrm{e} 1.3$ ), e, pela equação 1.5 :

$$
\tan \theta=\frac{u^{\prime}}{\gamma v}
$$

o que significa que $\theta<\theta^{\prime}$. É importante notar que, se a partícula for um fóton, $u^{\prime}=c$ e, 
no regime ultrarrelativístico $(v \sim c)$, temos

$$
\theta \approx \frac{1}{\gamma}
$$

Em resumo, um corpo que emite radiação isotropicamente em seu referencial de repouso (uma estrela na origem de $K^{\prime}$, por exemplo), possuirá emissão anisotrópica, concentrada na direção da velocidade relativa $v$ entre os dois referenciais, no referencial $K$. A figura 1.8 representa este fenômeno.
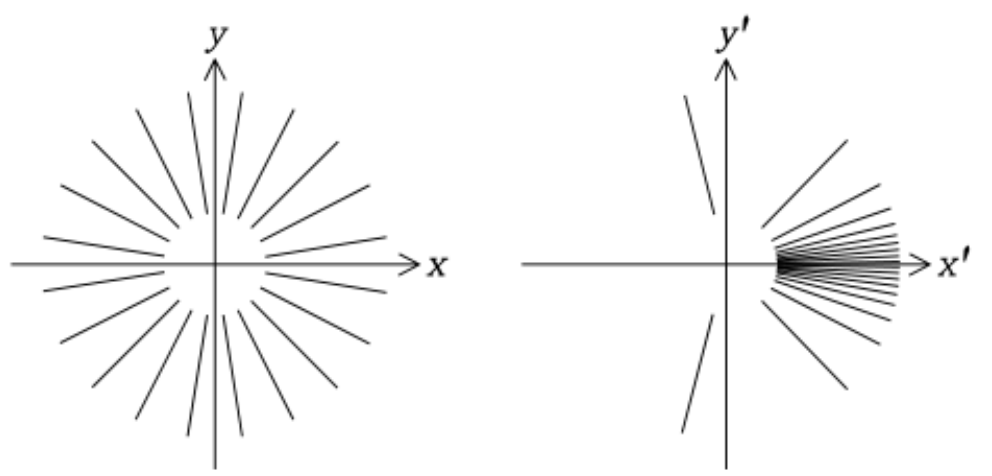

Figura 1.8: aberração relativística de um campo de radiação isotrópico (esquerda) visto pelo referencial de uma partícula em movimento com $\gamma=5$ (direita). Fonte: InverseCompton Scattering - http://www.cv.nrao.edu/course/astr534/InverseCompton. html. Acesso em 03/02/2016.

\subsubsection{Efeito Doppler}

Considerando uma fonte de radiação em movimento, no referencial $K$, que emite um período de radiação enquanto desloca-se do ponto 1 até o ponto 2 (figura 1.9) com velocidade $v$. Se a frequência da radiação no referencial de repouso, $K^{\prime}$, da fonte é $\omega^{\prime}$, então o tempo decorrido para a fonte mover-se do ponto 1 até o ponto 2 no referencial do observador é dado pelo efeito de dilatação temporal

$$
\Delta t=\gamma \Delta t^{\prime}=\frac{2 \pi \gamma}{\omega^{\prime}}
$$

Pela figura 1.9, notamos que $l=v \Delta t$ e $d=v \Delta t \cos \theta$. Entretanto, a diferença nos tempos de chegada $\Delta t_{c h}$ da radiação emitida nos pontos 1 e 2 é igual a $\Delta t$ menos o tempo que a luz levou para percorrer a distância $d$. Temos, então

$$
\Delta t_{c h}=\Delta t-\frac{d}{c}=\Delta t\left(1-\frac{v}{c} \cos \theta\right)
$$


A frequência $\omega$ observada será

$$
\omega=\frac{2 \pi}{\Delta t_{c h}}=\frac{\omega^{\prime}}{\gamma\left(1-\frac{v}{c} \cos \theta\right)} \longrightarrow \nu=\frac{\nu^{\prime}}{\gamma\left(1-\frac{v}{c} \cos \theta\right)}
$$

Onde o termo $\gamma^{-1}$ é um efeito puramente relativístico, enquanto que o fator $1-(v / c) \cos \theta$ aparece mesmo na mecânica newtoniana.

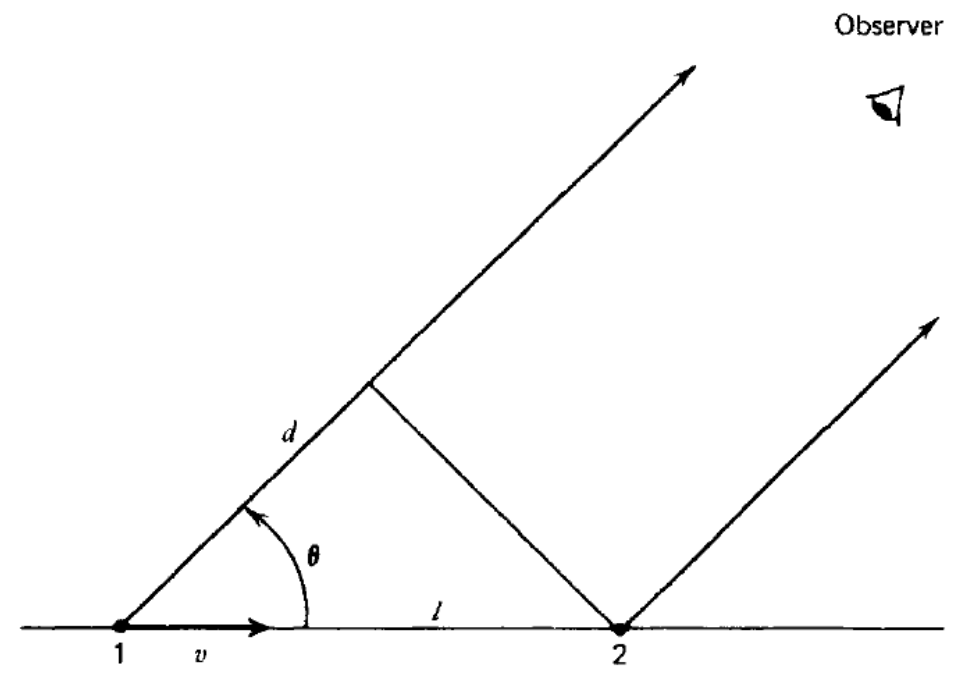

Figura 1.9: fonte em movimento causando o efeito Doppler. Fonte: Rybicki and Lightman (2008).

Para fontes com movimento relativístico na direção e sentido da linha de visada (como o jato dos blazares), temos $\cos \theta \approx 1$ e $(1-v / c) \approx 1 / 2 \gamma^{2}$ (ver equação 1.15), fazendo com que $\nu$ assuma valores arbitrariamente altos, muitas vezes na região dos raios $\gamma$.

\subsubsection{Síncrotron}

Partículas aceleradas por um campo magnético $\vec{B}$ emitem radiação. Para velocidades não-relativísticas, a descrição deste fenômeno é bastante simples e recebe o nome de radiação cíclotron. Sua frequência de emissão é simplesmente a frequência de giro da partícula no campo $\vec{B}$.

Entretanto, para partículas no regime relativístico, o espectro de frequências é muito mais complexo e estende-se muito além da frequência de giro. Este fenômeno é conhecido como radiação síncrotron.

A equação do movimento para uma partícula relativística com velocidade $v$ e carga $q$ sob a ação de um campo magnético uniforme e estático $\vec{B}$ é

$$
\frac{d(\gamma m \vec{v})}{d t}=\frac{q}{c} \vec{v} \times \vec{B}
$$

Mas como a força magnética não aumenta a velocidade da partícula, $\gamma$ é independente do 
tempo e $d(\gamma m \vec{v}) / d t=\gamma m d \vec{v} / d t$. Temos, então:

$$
\frac{d \vec{v}}{d t}=\frac{q \vec{v} \times \vec{B}}{\gamma m c} .
$$

Separando $\vec{v}$ em suas componentes paralela e perpendicular a $\vec{B}$, obtemos:

$$
\frac{d \vec{v}_{\|}}{d t}=0 \longrightarrow \vec{v}_{\|}=c t e
$$

$\mathrm{e}$

$$
\frac{d v_{\perp}}{d t}=\frac{q v_{\perp} B \sin (\pi / 2)}{\gamma m c},
$$

cujas soluções descrevem um movimento em forma de hélice em torno das linhas de campo magnético (figura 1.10). Como a aceleração centrípeta pode ser escrita em termos da frequência angular $\omega\left(a_{\perp}=d v_{\perp} / d t=v_{\perp}^{2} / R=v_{\perp} \omega\right)$, podemos reescrever a equação 1.9 como

$$
\omega=\frac{q B}{\gamma m c}=\frac{\omega_{c i c}}{\gamma}
$$

onde $\omega_{c i c}$ é a frequência de cíclotron.

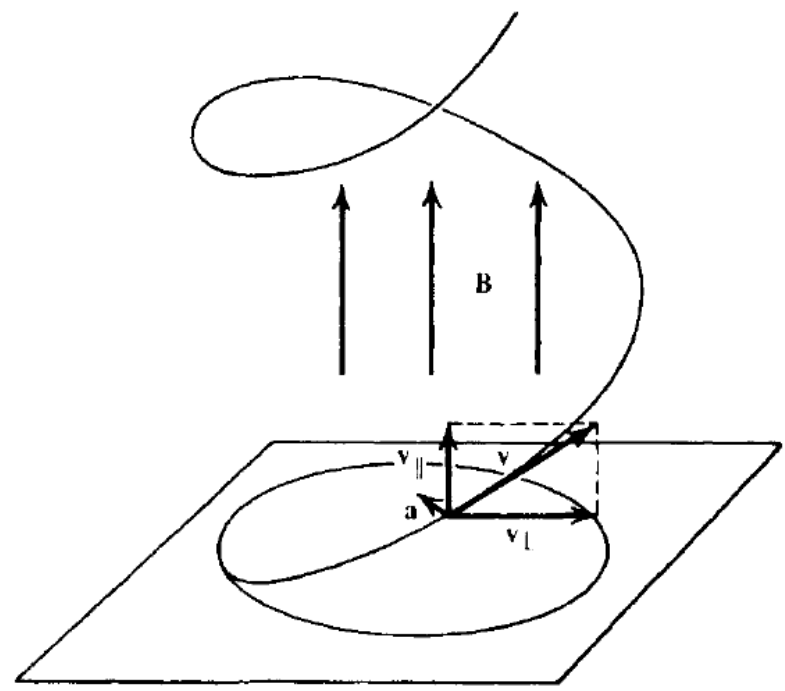

Figura 1.10: movimento de uma partícula carregada sob a ação de um campo magnético. Fonte: Rybicki and Lightman (2008).

A partícula acelerada emite radiação de acordo com a fórmula de Larmor (Rybicki and Lightman, 2008) a uma taxa

$$
P=\frac{2 q^{2} \gamma^{4} a_{\perp}^{2}}{3 c^{3}}=\frac{2 q^{2} \gamma^{4}}{3 c^{3}} \frac{q^{2} B^{2} v_{\perp}^{2}}{\gamma^{2} m^{2} c^{2}}
$$

Como $P \propto m^{-2}$, a potência emitida por partículas de alta massa, como prótons, é desprezível se comparada à emitida por elétrons. A emissão de síncrotron para um elétron 
espiralando num campo magnético é, então

$$
P=\frac{2}{3} r_{0}^{2} c \beta_{\perp}^{2} \gamma^{2} B^{2}
$$

Onde definimos $\beta_{\perp}=v_{\perp} / c$ e $r_{0}=q^{2} / m_{e} c^{2}$ como o raio clássico do elétron. A emissão é maior que a esperada no regime não-relativístico por um fator de $\gamma^{2}$.

\section{Espectro de síncrotron}

Por conta do efeito de beaming, a radiação emitida pelas partículas é concentrada na direção do seu vetor velocidade (figura 1.11).

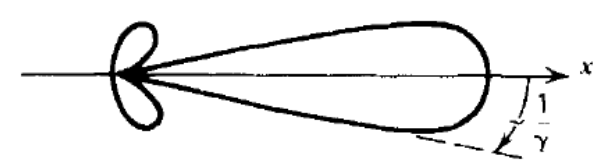

Figura 1.11: distribuição angular da radiação emitida por uma partícula com aceleração perpendicular à velocidade. Fonte: Rybicki and Lightman (2008).

O observador da figura 1.12 verá o pulso de radiação entre os pontos 1 e 2, enquanto o cone de radiação está em sua linha de visada. A duração do pulso no referencial do observador é

$$
\tau=\frac{\Delta \theta}{\omega}=\frac{2 / \gamma}{\omega}
$$

Mas por conta do efeito de retardo, o tempo de chegada $\tau_{c h}$ é menor que $\tau$. Temos, então

$$
\tau_{c h}=(1-\beta) \tau
$$

Substituindo a equação (1.13) em (1.14) e assumindo que em casos ultrarrelativísticos $\beta \approx 1 \mathrm{e}$

$$
\frac{1}{\gamma^{2}}=1-\beta^{2}=(1+\beta)(1-\beta) \approx 2(1-\beta) \longrightarrow(1-\beta) \simeq \frac{1}{2 \gamma^{2}},
$$

temos:

$$
\tau_{c h}=\frac{1}{\gamma^{3} \omega}
$$

O intervalo de tempo observado para cada pulso é menor que o período de giro por um fator de $\gamma^{3}$. O observador verá um pulso de radiação confinado num intervalo de tempo muito menor que o período de giro. O espectro será, então, espalhado por uma região extensa. Esta é uma característica essencial da radiação síncrotron. O pulso e seu espectro de potência são mostrados na figura 1.13. Diferente do espectro de potência da radiação cíclotron (que possui apenas um pico), obtemos uma série de picos muito próximos que, na prática, formam um espectro contínuo.

Em fontes astrofísicas, no entanto, observamos a emissão de síncrotron de populações 


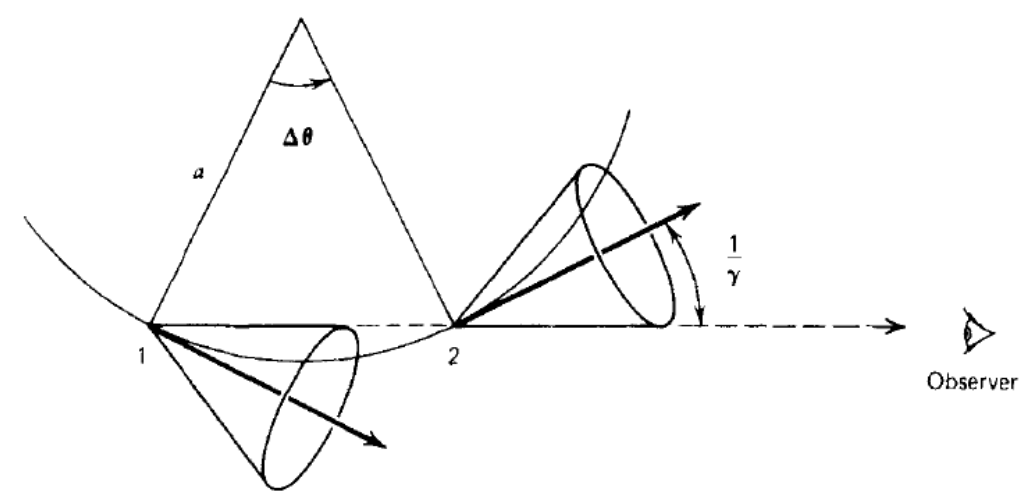

Figura 1.12: cone de emissão de síncrotron com o passar do tempo. Fonte: Rybicki and Lightman (2008).
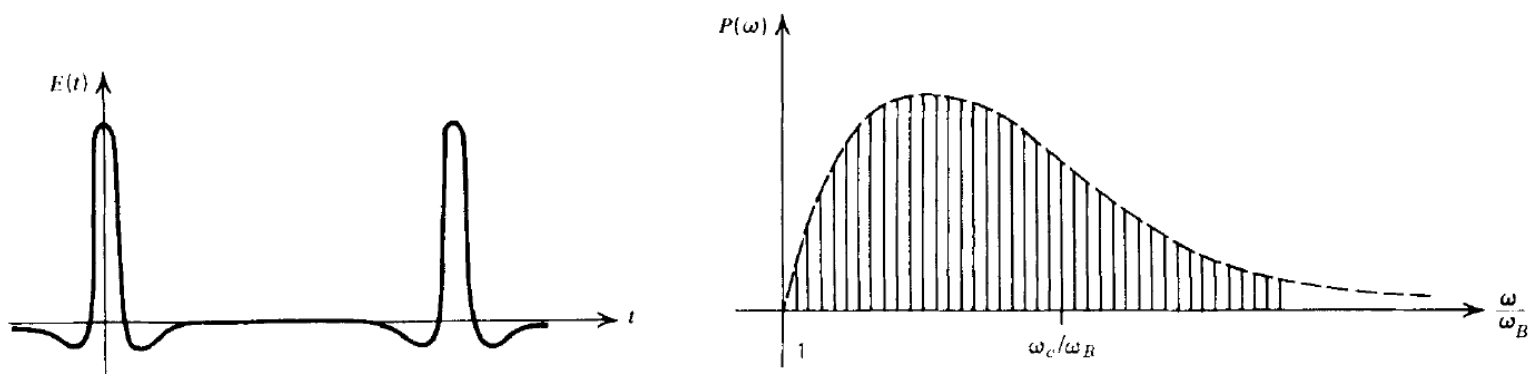

Figura 1.13: esquerda: dependência temporal do campo elétrico em pulsos de radiação síncrotron. Direita: espectro de potência do gráfico à esquerda. Fonte: Rybicki and Lightman (2008).

inteiras de elétrons, e não de apenas uma partícula. Os elétrons destas nuvens possuem velocidades e orientações distintas com relação ao campo magnético, fazendo com que o espectro de síncrotron observado seja a soma das contribuições individuais de cada elétron.

O espectro resultante está ilustrado na figura 1.14 .

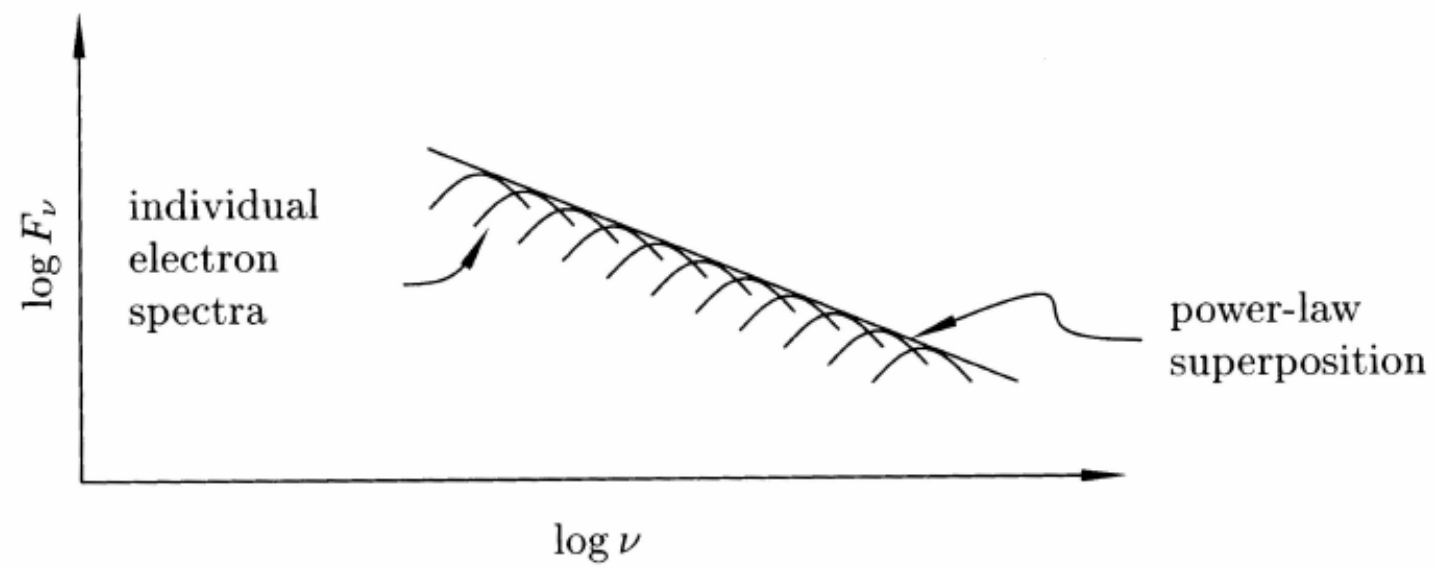

Figura 1.14: emissão de síncrotron por uma nuvem de elétrons. Fonte: Shu (1991). 


\subsubsection{Espalhamento Compton inverso}

Sempre que um elétron possui alta energia cinética comparada à energia de um fóton incidente, sua energia poderá ser transferida para o fóton. Este processo é conhecido como espalhamento Compton inverso. Nesta subseção assumiremos que no referencial de repouso do elétron $h \nu \ll m c^{2}$, então a correção relativística de Klein-Nishina para a seção de choque pode ser negligenciada (o principal efeito desta correção é reduzir a seção de choque do seu valor clássico à medida em que a energia do fóton incidente aumenta).

Vamos supor um campo de radiação isotrópico no referencial $K$, onde um elétron $e^{-}$ move-se em regime ultrarrelativístico. O campo de radiação no referencial $K^{\prime}$, onde o $e^{-}$ encontra-se em repouso, é bastante anisotrópico (figura 1.8). O efeito de beaming faz com que a maior parte dos fótons aproxime-se por um ângulo $\theta^{\prime} \sim \gamma^{-1}$ (subseção 1.2.1). O espalhamento Thomson desta radiação altamente anisotrópica sistematicamente reduz a energia cinética do $e^{-}$e a converte em radiação (inverse Compton radiation).

Para um elétron ultrarrelativístico $\left(\operatorname{com} \vec{\beta} \approx(1,0,0)=\beta_{x}\right.$ ) interagindo com um fóton que aproxima-se pelo eixo $\mathrm{x}(\operatorname{com} \cos \theta=1)$, a frequência da radiação que o atinge em seu referencial de repouso é dada pela inversa da equação (1.7):

$$
\nu_{0}^{\prime}=\nu_{0}[\gamma(1+\beta \cos \theta)] \approx 2 \gamma \nu_{0}
$$

No referencial de repouso do $e^{-}$, o espalhamento Thomson espalha o fóton sem mudar sua frequência $\left(\nu_{e s p}=\nu_{0}^{\prime}\right)$. No referencial do observador, o efeito beaming faz com que o fóton seja espalhado preferencialmente na direção do movimento do elétron e sua frequência é observada com um blueshift descrito por

$$
\nu_{o b s}=\nu_{e s p}[\gamma(1+\beta \cos \theta)]
$$

onde, mais uma vez, $\beta \approx 1$ e $\cos \theta \approx 1$. Assim:

$$
\nu_{o b s} \approx \nu_{e s p} 2 \gamma \approx 4 \gamma^{2} \nu_{0}
$$

No caso, $\nu_{\text {obs }}$ representa a frequência máxima da radiação espalhada no referencial do observador.

\section{Synchrotron self-Compton}

O espalhamento Compton inverso da radiação síncrotron pelos mesmos elétrons relativísticos que emitiram a radiação síncrotron é conhecido como Synchrotron self-Compton. O formato do espectro do espalhamento Compton inverso é similar ao de síncrotron independentemente do espectro da radiação incidente (mesmo que o espectro da radiação incidente seja de bremsstrahlung ou corpo negro). O pico de SSC é comumente encon- 
trado na spectral energy distribution (SED) de blazares (figura 1.15) na região dos raios $\mathrm{X}$ ou raios $\gamma$ (Konopelko et al., 2003).

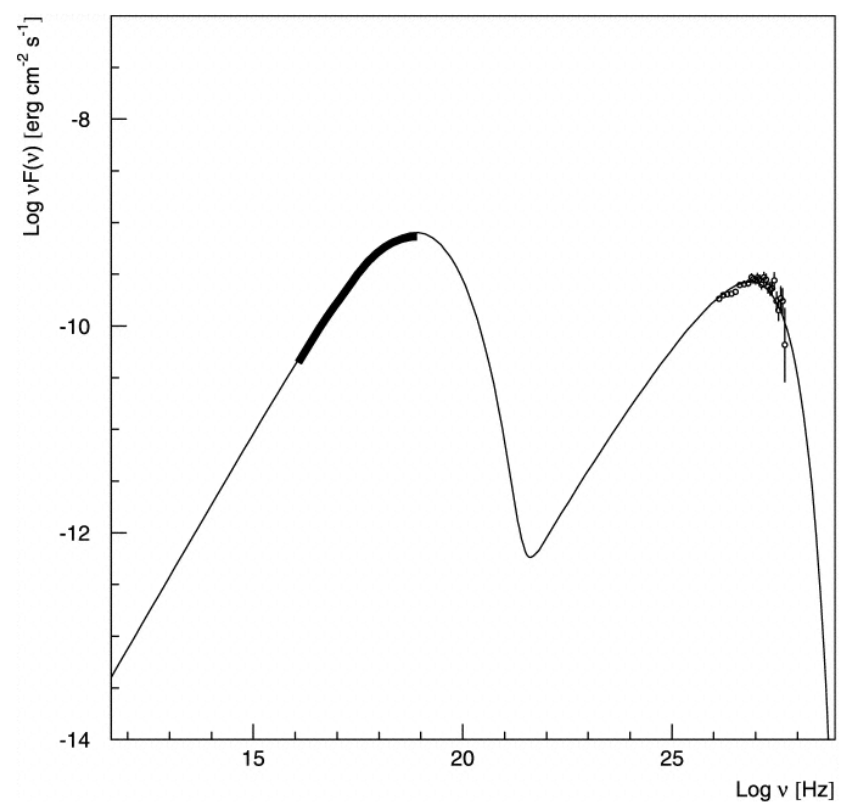

Figura 1.15: picos de síncrotron $\left(\sim 10^{19} \mathrm{~Hz}\right)$ e SSC $\left(\sim 10^{27} \mathrm{~Hz}\right)$ da SED do Mkn501. Fonte: Konopelko et al. (2003).

\section{$1.4 \quad \mathrm{AGNs}$}

A luz de galáxias normais no óptico e infravermelho próximo é dominada por estrelas, com pequena contribuição por gás e poeira. O espectro destas galáxias é razoavelmente bem descrito como uma superposição de espectros estelares, onde a temperatura superficial das estrelas pode assumir valores num intervalo relativamente pequeno. Como uma aproximação grosseira, a distribuição de luz de uma galáxia pode ser descrita pela superposição do espectro de Planck num intervalo de temperaturas que cobre aproximadamente uma década (de $\sim 3000 \mathrm{~K}$ a $\sim 40000 \mathrm{~K}$ ) (Schneider, 2014). Como o espectro de Planck possui uma distribuição de energia bastante estreita em torno do seu máximo em $h \nu \sim 3 k_{B} T$, o espectro de uma galáxia comum é basicamente confinado num intervalo entre $\sim 4000 \AA \mathrm{e} \sim 20000 \AA$.

Entretanto, algumas galáxias possuem espectro que se estende por um intervalo de energia muito maior. Muitas destas apresentam emissão significativa do rádio até os raios $\gamma$ (figuras 1.15 e 1.16). Esta emissão tem origem numa região central e compacta da galáxia, chamada de active galactic nuclei (AGN) e não pode ser explicada por fenômenos térmicos.

Algumas classes de AGNs, em particular os quasares, são observadas até redshifts $z \sim 6$. A luminosidade dos quasares pode exceder a de galáxias comuns por um fator de $\sim 10^{3}$, sendo emitida por uma região compacta com raio $\lesssim 1$ pc (Schneider, 2014). 


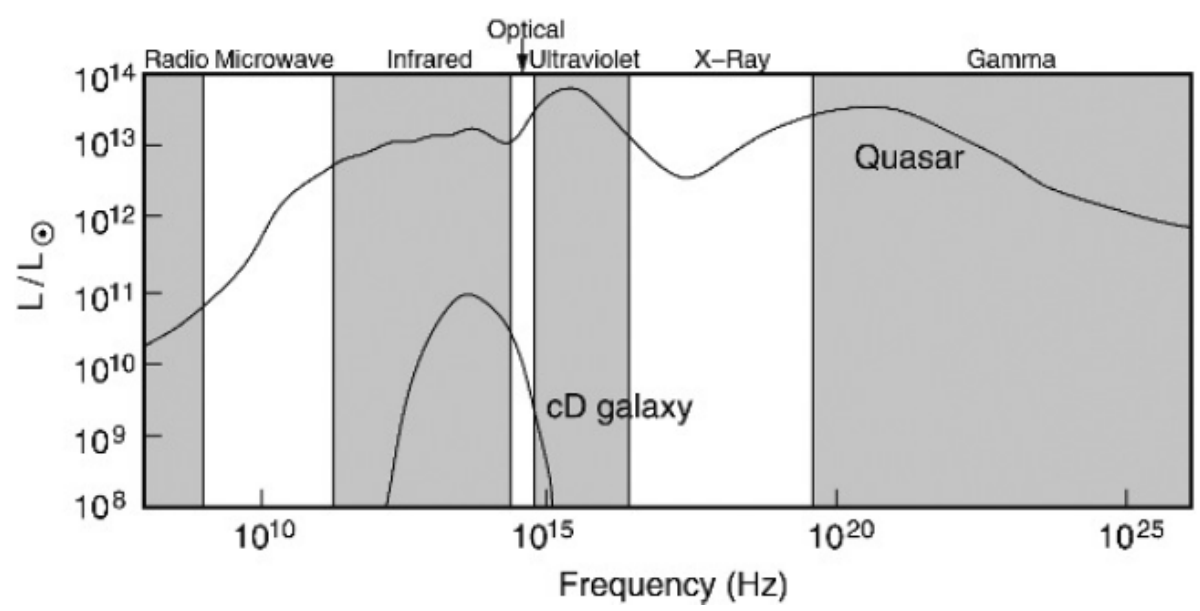

Figura 1.16: SED do quasar 3C273 em comparação ao de uma galáxia elíptica. Enquanto a radiação da galáxia elíptica abrange um intervalo de cerca de duas décadas em frequência, a emissão do quasar, que não pode ser interpretada como a superposição de espectros estelares, é observada desde o rádio até o gama. Fonte: Schneider (2014).

Quase todas as fontes de raios $\gamma$ extragalácticas observadas pelo Fermi são AGNs. Dentre estas fontes, o BL Lac PG 1553+113, o FSRQ 3C279, as galáxias Seyfert M88 e NGC1275, a rádio-galáxia M87 e vários LINERs foram analisados neste trabalho. Uma discussão destes tipos de núcleo ativo, assim como das emissões difusas da Via Láctea e isotrópica extragaláctica, será abordada nos tópicos seguintes.

\section{Radio loud e radio quiet}

É conveniente dividir os AGNs em duas classes, convencionalmente chamadas de radio loud e radio quiet. A emissão de objetos radio loud recebe contribuições tanto dos jatos quanto dos lóbulos. A combinação dessas emissões domina a luminosidade do AGN em rádio e possivelmente em outros comprimentos de onda. Objetos radio quiet são mais simples, já que a emissão dos jatos e lóbulos é desprezível em todos os comprimentos de onda.

Blazares, radio loud quasars e rádio-galáxias são exemplos de AGNs radio loud; enquanto que LINERs, Seyferts e radio quiet quasars (este último é basicamente uma versão mais luminosa das Seyfert, onde a distinção, apesar de arbitrária, costuma ser expressa em termos de alguma magnitude limite no óptico), pertencem à classe dos radio quiet.

\subsubsection{LINERs}

No universo local, os Low-ionization nuclear emission-line regions (LINERs) são encontrados em cerca de um terço de todas as galáxias mais brilhantes que $\mathrm{B}=15,5$ Netzer, 2013). Isso é maior que o número local de AGNs de alta ionização (como Seyferts e quasares) por um fator de 10 ou mais. LINERs são os AGNs de mais baixa luminosidade 
(Netzer, 2013) e comumente são chamados de low-luminosity AGNs (LLAGNs).

As fortes linhas de emissão no óptico no espectro dos LINERs incluem $\left[O_{I I I}\right] \lambda 5007$, $\left[O_{I I}\right] \lambda 3727,\left[O_{I}\right] \lambda 6300,\left[N_{I I}\right] \lambda 6584$ e linhas de Balmer do hidrogênio. Todas estas linhas são proeminentes em AGNs de alta ionização, mas, nos LINERs, suas intensidades relativas indicam um menor estado de ionização. Para fins comparativos, a razão das linhas $\left[O_{I I I}\right] \lambda 5007 / H_{\beta}$ em LINERs é $3 \sim 5$ vezes menor que em Seyferts 2 . Diagramas diagnóstico de linhas são ferramentas eficientes para separar LINERs de AGNs de alta ionização (figura 1.17).

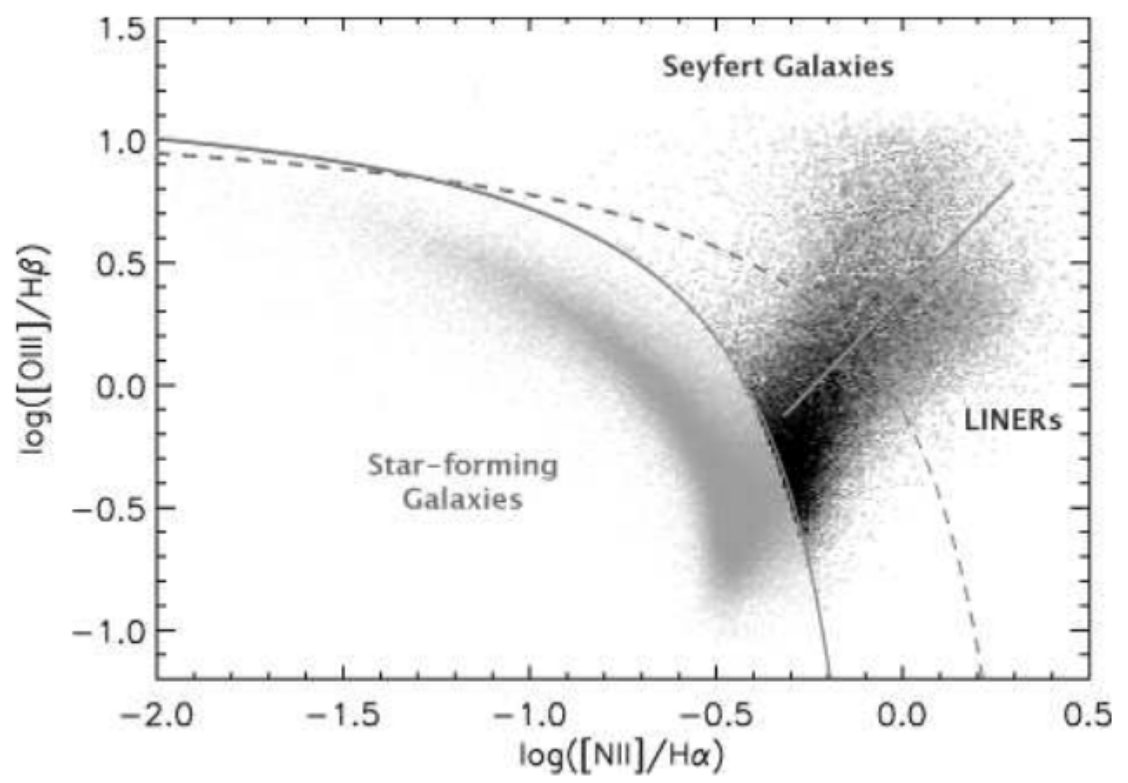

Figura 1.17: diagrama diagnóstico de uma amostra de galáxias do SDSS usando quatro linhas de emissão fortes: $\mathrm{H} \alpha, \mathrm{H} \beta,[O I I I] \lambda 5007$ e $[N I I] \lambda 6584$, para distinguir as galáxias que são dominadas por ionização de estrelas jovens das que são ionizadas por SEDs de AGNs. As curvas indicam as divisões teórica (pontilhada) e empírica (contínua) entre AGNs e galáxias com alta taxa de formação estelar. Fonte: Groves (2006).

É importante observar que espectros parecidos com os dos LINERs podem ser produzidos por vários mecanismos que não estão associados à atividade no buraco negro. Gás excitado por choques, com temperatura de elétrons $\sim 5 \times 10^{4} \mathrm{~K}$, podem resultar em linhas de emissão parecidas com as encontradas nos LINERs. Tal gás é encontrado nas regiões mais externas de sistemas em interação, como os ULIRGs (ultra luminous infrared galaxies), e em outros ambientes astronômicos associados a vários tipos de ventos. Uma maneira de distinguir o gás ionizado por choques do fotoionizado é determinar a temperatura dos elétrons do gás que está produzindo as linhas. Para um dado nível de ionização, a temperatura do gás fotoionizado é menor que a do gás ionizado por choques já que, para o primeiro caso, a ionização é consequência de fótons de alta energia (este processo depende da SED e do parâmetro de ionização. Não depende da temperatura do gás). Por outro lado, em um gás excitado por choques, a colisão entre partículas com 
alta velocidade requer altas temperaturas. Como a temperatura do gás pode ser medida diretamente por razões de linhas, estas medidas são a chave para entender a natureza da fonte ionizante (Netzer, 2013).

\subsubsection{Galáxias Seyfert}

Em muitos aspectos, galáxias Seyfert são similares a qualquer outra galáxia, exceto que suas regiões centrais mostram propriedades bastante peculiares. Possuem núcleo incrivelmente brilhante, com luminosidades que variam entre $10^{8}$ e $10^{11}$ luminosidades solares. Apenas cerca de 5\% delas são fontes brilhantes no rádio, e boa parte possui emissão moderada em raios X (Schneider, 2014).

Em imagens ópticas, galáxias Seyfert são identificadas com um núcleo incrivelmente brilhante (figura 1.18). De acordo com seu espectro, podemos classificar a Seyfert como do tipo 1 ou 2.

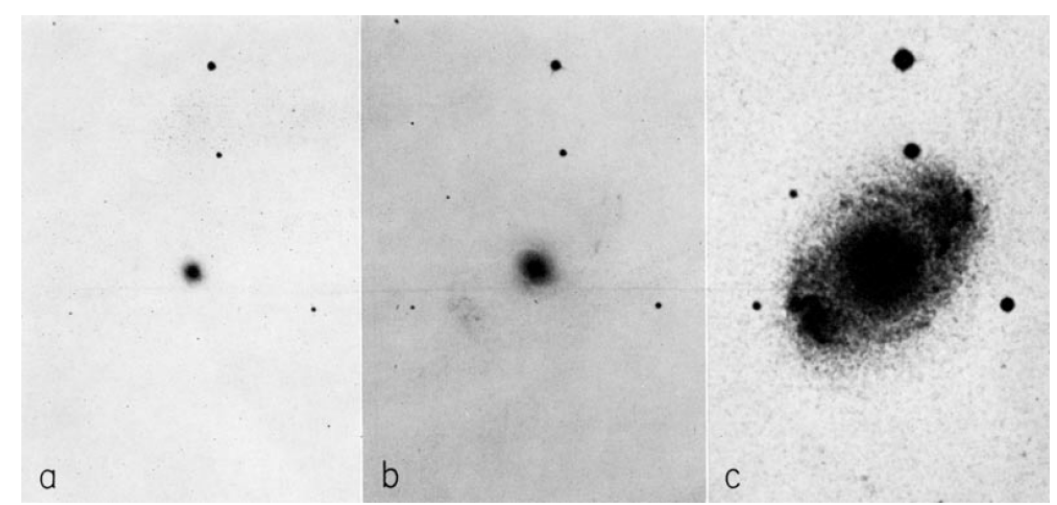

Figura 1.18: três imagens da galáxia Seyfert NGC4151, com o tempo de exposição aumentando da esquerda para a direita. O núcleo excepcionalmente brilhante, como uma de suas características principais, era bastante usado nas primeiras identificações de Seyferts. Fonte: Morgan (1968).

O tipo 1 caracteriza-se por possuir linhas largas e estreitas bastante proeminentes no seu espectro (onde "estreita", aqui, ainda significa centenas de quilômetros por segundo - são comumente mais largas que as linhas de emissão de galáxias comuns). Já o tipo 2 possui apenas linhas estreitas (figura 1.19).

\subsubsection{Rádio-galáxias}

A descoberta de rádio-galáxias precede a descoberta óptica de AGNs (Netzer, 2013). Ela remonta ao final dos anos 40 e início dos anos 50 (exceto pelo famoso artigo de Seyfert de 1943: Seyfert (1943)). Muitas destas fontes foram observadas posteriormente com espectro óptico-UV muito similar ao dos vários tipos de AGNs descobertos no óptico. Estas fontes apresentam estruturas em formato de lóbulos (duplo ou único), com dimensões que podem exceder em muito o tamanho de sua galáxia hospedeira, e núcleo com forte 

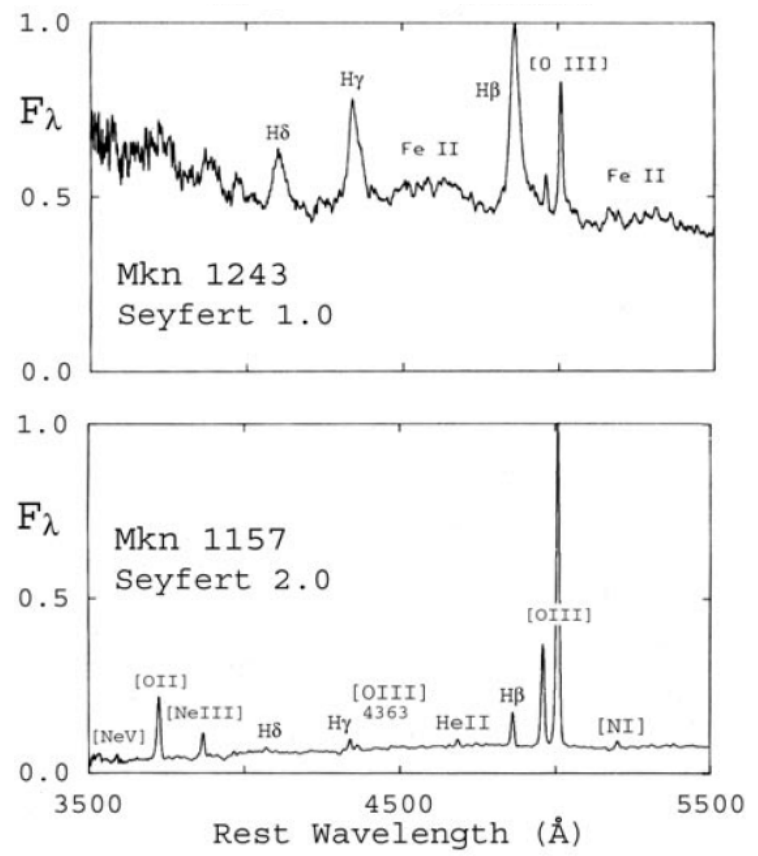

Figura 1.19: Diferenças no espectro das Seyfert. As linhas nomeadas entre colchetes são proibidas. Fonte: adaptado de Osterbrock (1984).

emissão em rádio coincidente com o centro óptico da própria galáxia (Netzer, 2013). A emissão em rádio destas galáxias radio loud é característica do efeito síncroton (seção 1.2), abrangendo um grande intervalo de frequências com inclinação suave e alta polarização (Schneider, 2014).

Fontes de rádio são divididas em duas classes, Fanaroff-Riley I (FR-I) e II (FR-II), cuja distinção é baseada na morfologia da emissão em rádio em larga escala: FR-I são mais brilhantes próximas ao núcleo, enquanto que FR-II são mais brilhantes em lóbulos afastados do núcleo (figura 1.20). Fanaroff e Riley (Fanaroff and Riley, 1974) observaram uma divisão de luminosidade entre estas duas classes: FR-I são pouco luminosas $\left(L_{\nu}(1,4\right.$ $\left.\mathrm{GHz}) \lesssim 10^{32} \mathrm{erg} \mathrm{s}^{-1} \mathrm{~Hz}^{-1}\right)$ e FR-II são muito luminosas $\left(L_{\nu}(1,4 \mathrm{GHz}) \gtrsim 10^{32} \mathrm{erg} \mathrm{s}^{-1}\right.$ $\mathrm{Hz}^{-1}$ ) (Schneider, 2014).

\subsubsection{Blazares}

Antes considerados como fontes raras, blazares, um tipo de AGN radio loud apontando seus jatos na direção do observador, são hoje bastante comuns. Cerca de 3000 já foram catalogados e são as fontes extragalácticas mais comuns em raios $\gamma$. No contexto deste projeto, alguns blazares foram usados para testar nosso código (Capítulo 2).

Definimos um blazar como um AGN que mostra uma ou mais das seguintes propriedades (Netzer, 2013):

- Emissão intensa e altamente variável em raios gama. 

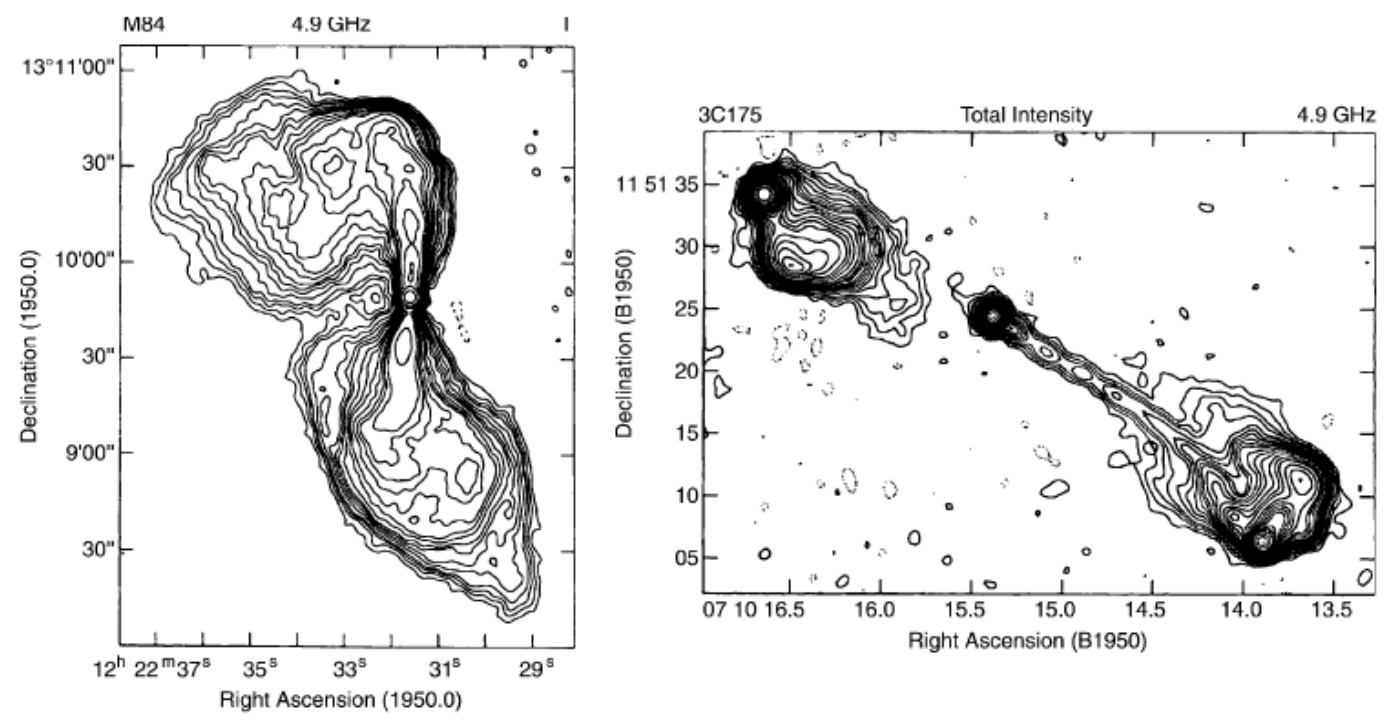

Figura 1.20: mapas em rádio $(\lambda=6 \mathrm{~cm})$ de duas rádio-galáxias: M84, à esquerda, é uma FR-I; 3C175, à direita, é uma FR-II. Galáxias FR-II geralmente possuem jatos, que apresentam estrutura interna e raramente são simétricos (às vezes, como é o caso, apenas um é observado). Fonte: Bridle et al. (1994) e Laing and Bridle (1987) .

- Emissão intensa e altamente variável em rádio associada a um espectro plano no rádio e, ocasionalmente, movimento superluminal.

- Jato em rádio, raios X, e/ou raios $\gamma$ com claras indicações de movimento relativístico.

- Uma SED com dois picos, um de baixa frequência centrado entre o rádio e os raios $\mathrm{X}$ e um de alta frequência centrado entre os raios $\mathrm{X}$ e raios $\gamma$ (figura 1.21).

- Emissão muito fraca de linhas estreitas e/ou largas, indicando fotoionização por uma fonte não estelar de radiação.

\section{Beaming relativístico}

O movimento relativístico do jato na direção do observador dá origem aos fenômenos de beaming, onde o brilho observado de uma fonte depende da sua velocidade em relação ao observador, e Doppler shift, que faz com que o fluxo medido em determinada frequência $\nu_{0}$ seja diferente de acordo com o referencial em que o observador se encontra.

De acordo com a relatividade especial, uma fonte em movimento que emite isotropicamente em seu referencial de repouso possui um padrão de emissão anisotrópico com uma distribuição angular que depende de sua velocidade em relação ao observador. A radiação é emitida preferencialmente na direção do vetor velocidade da fonte, então a fonte parecerá mais brilhante se estiver movendo-se na direção do observador (figura 1.22). 


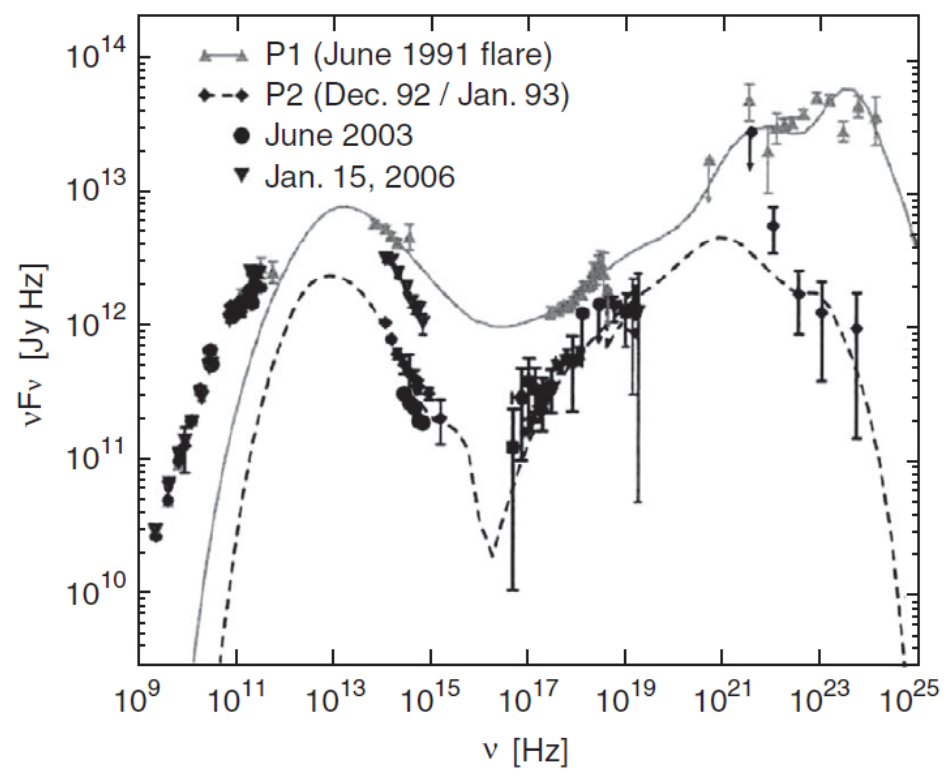

Figura 1.21: SED do blazar 3C 279 em épocas diferentes, mostrando os dois picos característicos em baixas (atribuido à radiação síncrotron) e altas energias (atribuído ao efeito Compton inverso - SSC) e a variabilidade da fonte. Fonte: Böttcher et al. (2007).
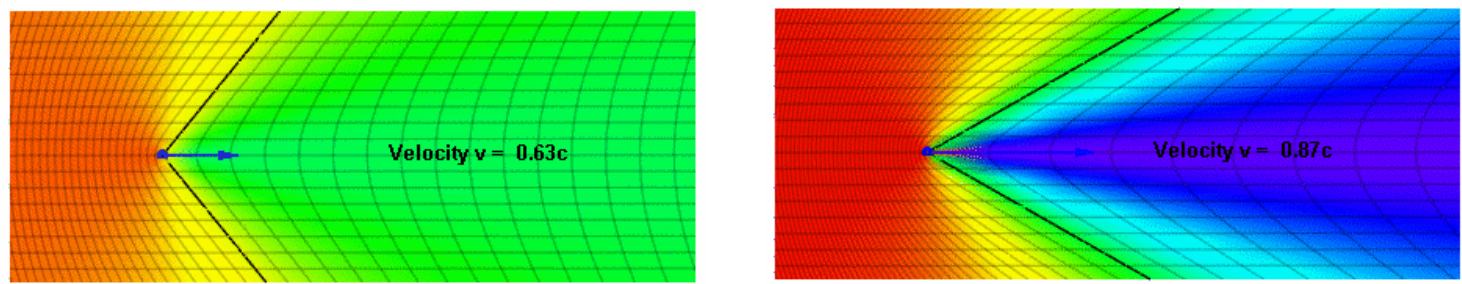

Figura 1.22: demonstração do efeito Doppler e beaming relativístico. A radiação é emitida preferencialmente na direção e sentido de $v$ (cone formado pelas linhas pretas). Fonte: Relativistic Doppler effect - TxAlien, Creative Commons - https://en.wikipedia.org/ wiki/Relativistic_Doppler_effect. Acesso em 28/01/2016. 
Vimos, na seção 1.2, que devido ao efeito Doppler a frequência $\nu$ detectada pelo observador é

$$
\nu=\frac{\nu^{\prime}}{\gamma(1-\beta \cos \phi)}
$$

onde $\gamma$ é o fator de Lorentz, $\beta=v / c$ e $\phi$ é o ângulo entre o vetor velocidade da fonte e a linha de visada. É sabido (Rybicki and Lightman, 2008) que $I / \nu^{3}$ é um invariante de Lorentz, o que significa que, no referencial do observador, o efeito Doppler causará um aumento na intensidade da fonte de acordo com

$$
\frac{I}{\nu^{3}}=\frac{I^{\prime}}{\nu^{\prime 3}} \rightarrow \frac{I \gamma^{3}(1-\beta \cos \phi)^{3}}{\nu^{\prime 3}}=\frac{I^{\prime}}{\nu^{\prime 3}}
$$

onde substituímos $\nu$ pelo seu valor na equação 1.20 , e consequentemente:

$$
I=\left(\frac{1}{\gamma(1-\beta \cos \phi)}\right)^{3} I^{\prime} \equiv D_{+} I^{\prime}
$$

onde $D_{+}$é definido como fator de Doppler. Por conta destes efeitos, a radiação do jato relativístico pode ser altamente amplificada.

Uma outra consequência do beaming é que se um segundo jato existir e mover-se na direção oposta ao observador, sua intensidade será atenuada por um fator

$$
D_{-}=\left(\frac{1}{\gamma(1+\beta \cos \phi)}\right)^{3}
$$

relativo ao referencial de repouso da fonte $\left(D_{-}\right.$pode ser obtido a partir de $D_{+}$se trocarmos $\phi$ por $\phi+\pi$, já que o segundo jato move-se na direção oposta). A razão entre as equações (1.21) e 1.22 para jatos relativísticos costuma ser bastante elevada e é a explicação canônica para as observações de fontes com um jato só feitas pelo VLBI (Schneider, 2014). Este efeito é conhecido como favoritismo Doppler.

A radiação dos jatos pode ofuscar todo o resto da emissão do AGN, já que $D_{+}$pode assumir valores bastante elevados. O efeito de beaming também consegue explicar a rápida variabilidade de blazares: se a velocidade do material que está emitindo for próxima da velocidade da luz, pequenas mudanças na velocidade do jato ou na sua direção já podem mudar notavelmente o fator de Doppler $D_{+}$.

\section{Dois tipos de blazares: BL Lac e FSRQ}

Apesar de todos os blazares compartilharem as mesmas propriedades de variabilidade e emissão não-térmica em todo o espectro, eles também apresentam diversidade. Os blazares são divididos em dois grupos principais, cujas maiores diferenças estão nas suas propriedades ópticas: (i) Flat Spectrum Radio Quasars (FSRQ), que apresentam fortes linhas largas de emissão no óptico, e (ii) BL Lacs, cujo espectro geralmente apresenta 
linhas fracas de emissão, algumas vezes linhas de absorção e, em alguns casos, pode ser completamente desprovido linhas (figura 1.23).
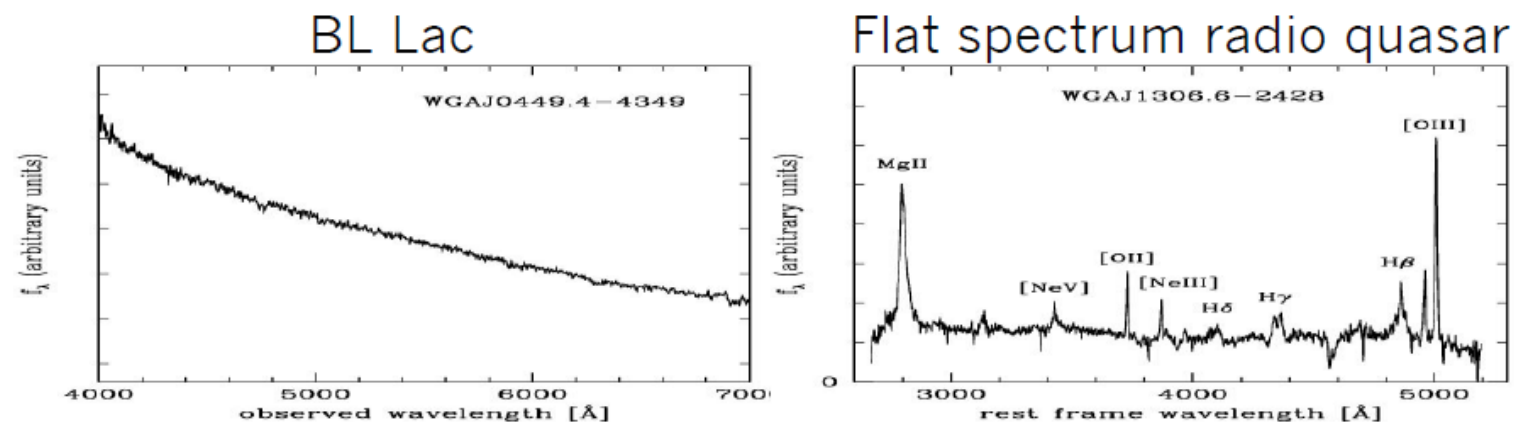

Figura 1.23: espectro óptico de um BL Lac e um FSRQ. Fonte: Padovani et al. (2012).

A classificação de blazares depende de suas características ópticas, onde estes emitem uma mistura de três tipos de radiação: (i) radiação não-térmica relacionada ao jato; (ii) radiação térmica originada da acreção de matéria no buraco negro supermaciço e da broad line region; e (iii) luz da galáxia hospedeira (Giommi et al., 2012). A figura 1.24 destaca estas três componentes sobrepostas à spectral energy distribution de quatro blazares bem conhecidos.

As principais diferenças entre as duas classes são:

- espectro óptico (por definição). Entretanto, existem alguns objetos na transição entre BL Lac e FSRQ (que inclui o próprio BL Lacertae, o protótipo dos BL Lacs).

- distribuição de redshift. FSRQs são tipicamente encontradas em redshifts $\sim 1-2$ e até $\sim 5,5$, enquanto que os BL Lacs são geralmente muito mais próximos, com pouquíssimos casos em $z>0,6$. Devemos destacar, porém, que não conseguimos medir o redshift de grande parte dos BL Lacs, por conta da ausência de linhas no espectro.

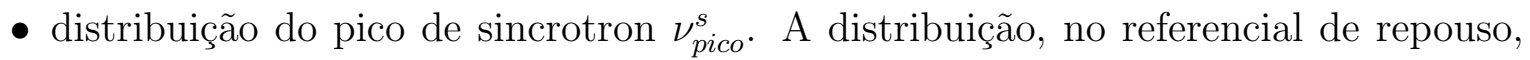
do $\nu_{\text {pico }}^{s}$ de FSRQs é centrada em baixas energias $\left(\left\langle\nu_{\text {pico }}^{s}\right\rangle=10^{13.1 \pm 0.1} \mathrm{~Hz}\right)$, enquanto que a distribuição de $\nu_{\text {pico }}^{s}$ para os BL Lacs é maior por, no mínimo, uma ordem de magnitude.

\subsubsection{Buracos negros e discos de acreção}

Antes de concluirmos que a "força motriz" por trás da geração de energia em AGNs são buracos negros, alguns fatos observacionais relevantes devem ser notados (Schneider, 2014): 

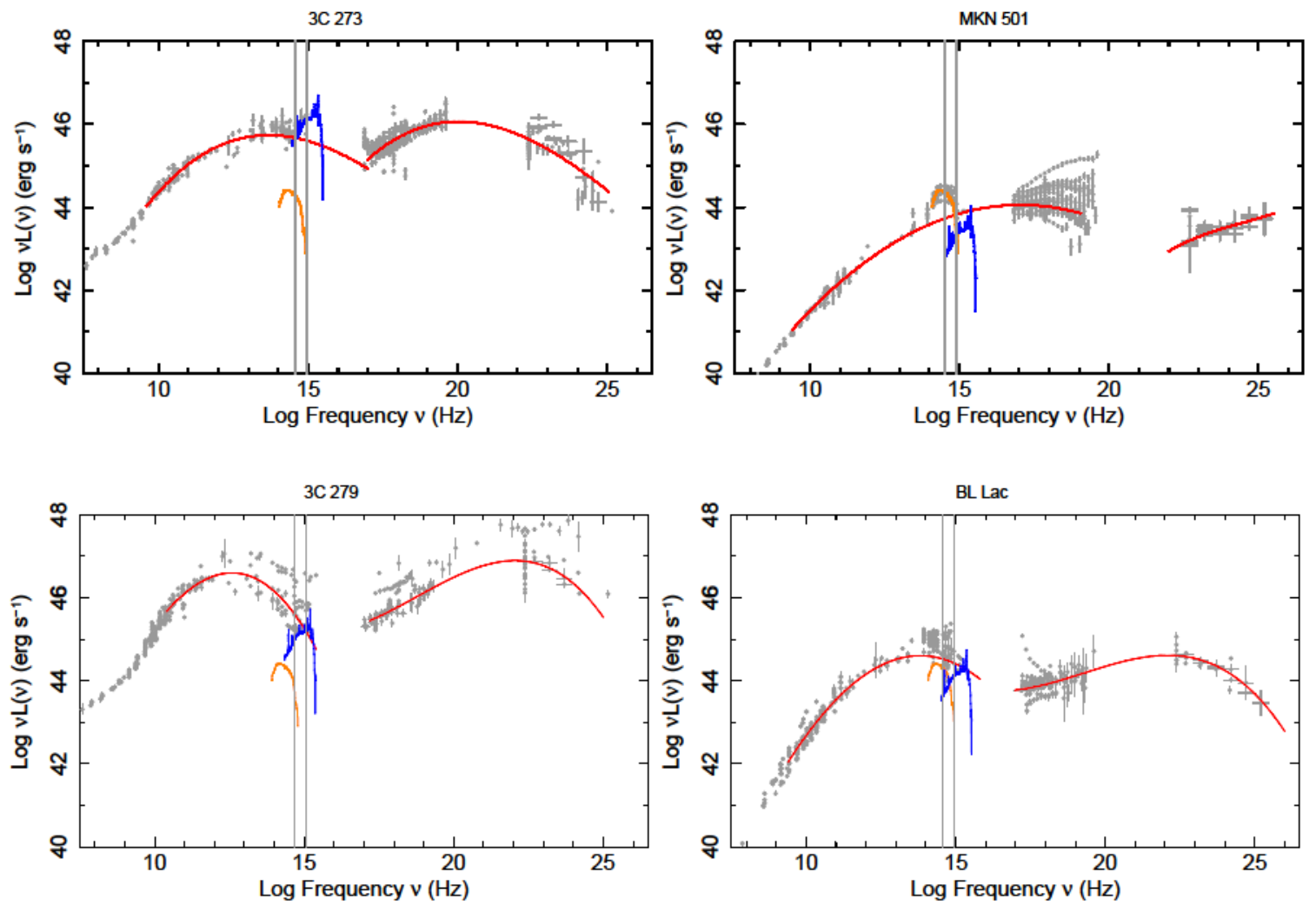

Figura 1.24: SEDs de quatro blazares: dois FSRQs (3C 273 e 3C 279) e dois BL Lacs (MKN 501 e BL Lac). A linha vermelha representa a radiação não-térmica emitida pelo jato; a linha azul representa a emissão do disco de acreção e da broad line region; e a linha laranja representa a luz da galáxia hospedeira. As duas linhas verticais indicam a janela do ótico $(3800-8000 \AA)$. Fonte: Giommi et al. (2012). 
- A extensão de algumas fontes de rádio em AGNs pode atingir mais de $1 \mathrm{Mpc}$. Para esta escala de tamanho, um tempo de vida mínimo para a atividade no núcleo desses objetos pode ser derivado, já que, mesmo se a região emissora de rádio expandir-se para longe do núcleo com a velocidade da luz, a idade da fonte seria $t \gtrsim 10^{7}$ anos.

- Alguns quasares têm luminosidade de até $L_{b o l} \sim 10^{47} \mathrm{erg} / \mathrm{s}$. Assumindo que a luminosidade não muda substancialmente com a idade da fonte, a energia total emitida (grosseiramente estimada) durante a idade mínima destes objetos seria da ordem de

$$
E \gtrsim 10^{47} \mathrm{erg} / \mathrm{s} \times 10^{7} \text { anos } \sim 3 \times 10^{61} \mathrm{erg} .
$$

- A luminosidade de alguns AGNs varia mais de 50\% numa escala de tempo de um dia. A partir desta escala de tempo, um limite superior para a extensão espacial da fonte pode ser determinado $(d \approx 2 c t /(1+z)$, para objetos "face on", onde z é o redshift do alvo). O tamanho característico dessas regiões é da ordem de $R \lesssim 1$ dia-luz $\left(3 \times 10^{15} \mathrm{~cm}\right)$.

Combinando estas observações, somos levados a pensar que a produção de energia em AGNs deve ser de natureza gravitacional. Para testar esta hipótese, usaremos, antes, o método "clássico" mais eficiente de produção de energia: a fusão nuclear. Assumiremos, a princípio, que a produção de energia em AGNs é baseada em processos termonucleares.

Pela queima de hidrogênio em elementos mais pesados (indo até o ferro - o elemento com a maior energia de ligação por nucleon), cerca de $8 \mathrm{MeV} /$ nucleon são liberados (ou $0,008 m_{p} c^{2}$ por nucleon). A eficiência máxima deste processo é $\epsilon \lesssim 0,8 \%$, onde $\epsilon$ é definido como a fração de massa que é convertida em energia de acordo com $E=\epsilon m c^{2}$.

Para gerar $E=3 \times 10^{61}$ ergs por este mecanismo, a quantidade de massa necessária é

$$
m=\frac{E}{\epsilon c^{2}} \sim 4 \times 10^{42} g \sim 2 \times 10^{9} M_{\odot}
$$

onde usamos o valor estimado na equação (1.23). Se a energia de um AGN fosse produzida por fusão nuclear, toda a massa não convertida $[(1-\epsilon) m \approx m]$ deveria estar presente no seu núcleo.

Entretanto, o raio de Schwarzschild para esta quantidade de massa é

$$
r_{S}=\frac{2 G m}{c^{2}} \approx 6 \times 10^{14} \mathrm{~cm}
$$

ou seja, é da mesma ordem de grandeza do tamanho estimado da fonte. Este argumento demonstra que efeitos gravitacionais devem ter um papel crucial nestes núcleos ativos e que a hipótese de geração de energia por fusão nuclear é, no mínimo, inadequada, por conta de sua baixa eficiência $\epsilon$ e pelo fato de que um núcleo tão compacto (buraco negro) não poderia emitir qualquer radiação que (possivelmente) produzisse. O único mecanismo 
conhecido com maior eficiência é a transformação de energia potencial gravitacional em energia cinética.

A energia potencial da matéria caindo no buraco negro supermaciço (SMBH) é convertida em energia cinética. Se parte desta energia puder ser convertida em energia interna (calor) e subsequentemente ser emitida em forma de radiação, $\epsilon$ pode ser muito maior que em processos termonucleares. Da teoria de acreção em buracos negros, uma eficiência máxima de $\epsilon \sim 6 \%$ para um buraco negro sem rotação (Schwarzschild) é derivada. Um buraco negro com o máximo de momento angular permitido pode ter uma eficiência de até $\epsilon \sim 29 \%$ (Schneider, 2014).

De maneira informal, podemos facilmente estimar a eficiência deste processo: seja $d M / d t$ a taxa de acreção de matéria no buraco negro, e seja a luminosidade descrita pelas equações (aqui, consideramos que toda a energia liberada na acreção é convertida em radiação e que o aumento de massa do buraco negro é desprezível):

$$
L=\epsilon \frac{d M}{d t} c^{2}
$$

$\mathrm{e}$

$$
L=\frac{d|U|}{d t}
$$

temos

$$
\frac{G M_{B N}}{r} \frac{d M}{d t}=\epsilon \frac{d M}{d t} c^{2} \rightarrow \epsilon=\frac{G M_{B N}}{r c^{2}} .
$$

Substituindo o raio de Schwarzschild $r_{S}=2 G M_{B N} / c^{2}$, obtemos

$$
\epsilon=\frac{r_{S}}{2 r}
$$

onde $\mathrm{r}$ não pode assumir valores menores que $r_{S}$. Se $r \approx 5 r_{S}$, a eficiência do processo será $\epsilon \sim 0,1$.

\section{Acreção}

Através de fricção com outras partículas de gás e pela transferência de momento resultante, o gás em volta do buraco negro aquece e aglomera-se em um disco orientado perpendicularmente à direção do vetor momento angular. Adicionalmente, a mesma fricção causa a desaceleração da velocidade rotacional deste disco, fazendo com que o gás lentamente caia em direção ao centro (Schneider, 2014).

De acordo com o teorema do virial, metade da energia potencial liberada é convertida em energia cinética, enquanto que a outra metade é convertida em energia interna, que acarreta no aumento da temperatura do disco. 
Quando uma massa $m$ cai de um raio $r+\Delta r$ para $r$, a energia

$$
\Delta E=\frac{G M_{\bullet} m}{r}-\frac{G M_{\bullet} m}{r+\Delta r} \approx \frac{G M_{\bullet} m}{r} \frac{\Delta r}{r}
$$

é liberada. Aqui, $M_{\bullet}$ representa a massa do $\mathrm{SMBH}$ e os efeitos de auto-gravidade do disco são negligenciados. Metade desta energia é convertida em calor, $E_{\text {calor }}=\Delta E / 2$. Assumindo que esta energia é emitida localmente, a luminosidade correspondente é

$$
\Delta L=\frac{G M_{\bullet} \dot{m}}{2 r^{2}} \Delta r
$$

onde $\dot{m}$ é a taxa de acreção.

Se o disco for opticamente espesso, a emissão local corresponde à emissão de um corpo negro e, portanto, o anel entre $r$ e $r+\Delta r$ emite de acordo com

$$
\Delta L=2 \times 2 \pi r \Delta r \sigma_{S B} T^{4}(r)
$$

onde $\sigma_{S B}$ é a constante de Stefan-Boltzmann e o fator 2 aparece por levarmos em conta os dois lados do disco. Combinando as equações (1.24) e (1.25), obtemos a dependência radial da temperatura do disco:

$$
T(r)=\left(\frac{G M_{\bullet} \dot{m}}{8 \pi \sigma_{S B} r^{3}}\right)^{1 / 4}
$$

Uma derivação mais acurada considera explicitamente a dissipação por fricção, mas, exceto por um fator de correção numérico, o resultado é o mesmo (Schneider, 2014):

$$
T(r)=\left(\frac{3 G M_{\bullet} \dot{m}}{8 \pi \sigma_{S B} r^{3}}\right)^{1 / 4}
$$

que é válido para $r>>r_{S}$. Escalando $r$ com o raio de Schwarzschild, obtemos

$$
T(r)=\left(\frac{3 G M_{\bullet} \dot{m}}{8 \pi \sigma_{S B} r_{S}^{3}}\right)^{1 / 4}\left(\frac{r}{r_{S}}\right)^{-3 / 4} .
$$

Substituindo $r_{S}=2 G M_{\bullet} / c^{2}$ no primeiro parêntesis, a equação acima pode ser reescrita como

$$
T(r)=\left(\frac{3 c^{6}}{64 \pi \sigma_{S B} G^{2}}\right)^{1 / 4} \dot{m}^{1 / 4} M_{\bullet}^{-1 / 2}\left(\frac{r}{r_{S}}\right)^{-3 / 4} .
$$

A temperatura no disco decresce com o raio $\left(\propto r^{-3 / 4}\right)$, como esperado. Considerando que a emissão total do disco é, em primeira aproximação, a superposição de anéis de corpo negro com diferentes raios e diferentes temperaturas, o espectro resultante deve abranger um intervalo de energias muito mais largo que o espectro de Planck. Ainda notamos que, 
para uma razão $r / r_{S}$ fixa, a temperatura decresce com a massa do SMBH. O que implica dizer que a temperatura máxima de um disco é menor para SMBHs de maior massa. Esta conclusão é um tanto quanto inesperada, mas pode ser explicada pela diminuição das forças de maré em um raio fixo com o aumento de $M_{\bullet}$. Em particular, isto implica que a temperatura máxima do disco num AGN é muito menor que em discos de acreção em torno de fontes estelares. Discos de acreção em torno de estrelas de nêutrons e buracos negros de massa estelar emitem a maior parte da energia em raios X. Em contraste, AGNs emitem mais no UV (Schneider, 2014).

\subsubsection{Modelo unificado}

Apesar das diferentes classes de AGN parecerem bem distintas, muitas delas possuem propriedades em comum. Uma questão recorrente é se essas classes são intrinsecamente diferentes ou são intrinsecamente similares e apenas parecem ser diferentes. Dado que a estrutura básica de um AGN, formada por um SMBH e um disco de acreção, é axissimétrica, objetos similares podem parecer diferentes se vistos sob ângulos diferentes. Algumas das observações que suportam este modelo anisotrópico de emissão são a morfologia da emissão em rádio, com os lóbulos de rádio expandindo-se em direções privilegiadas, e os cones de ionização (figura 1.25) na narrow line region (NLR).

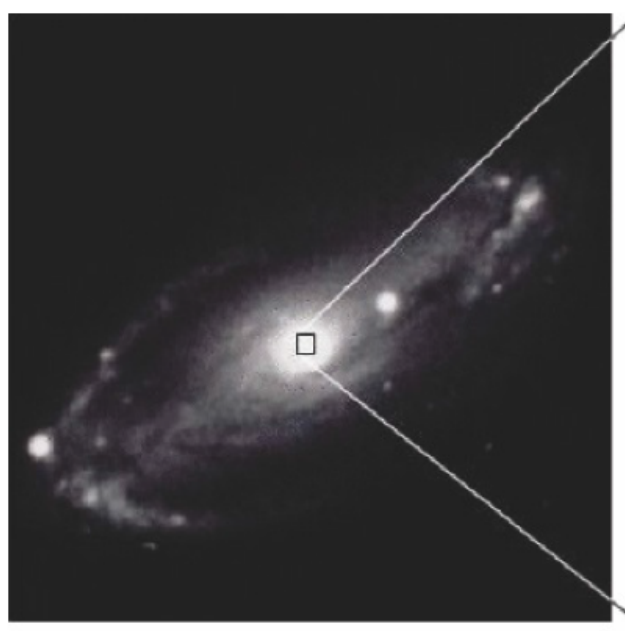

Ground View

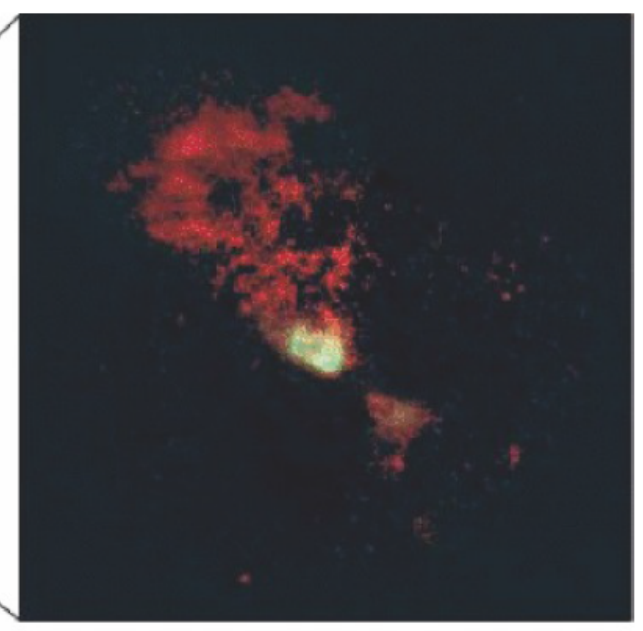

HST View

Figura 1.25: Seyfert NGC 5728. Esquerda: imagem em larga escala mostrando o disco da galáxia; direita: imagem do HST obtida com um filtro (narrow-band filter) centrado numa linha de emissão estreita. A imagem mostra claramente que a NLR não é esférica, mas consiste de dois cones. Desta observação, concluímos que a radiação ionizante do AGN não é isotrópica; é emitida em duas direções preferenciais perpendiculares ao disco da galáxia (e provavelmente perpendicular ao disco de acreção). Fonte: Allan Sandage e Andrew S. Wilson. Ver referência Schneider (2014) para mais detalhes.

A observação de linhas largas no espectro polarizado (figura 1.26) da galáxia NGC 1068, uma Seyfert 2, foi de crucial importância para suportar o modelo unificado. A 
galáxia deve ter uma broad line region (BLR), mas que só é visível em luz polarizada. Os fótons emitidos pela BLR sofrem espalhamento na poeira em torno do SMBH e são detectados como luz polarizada.
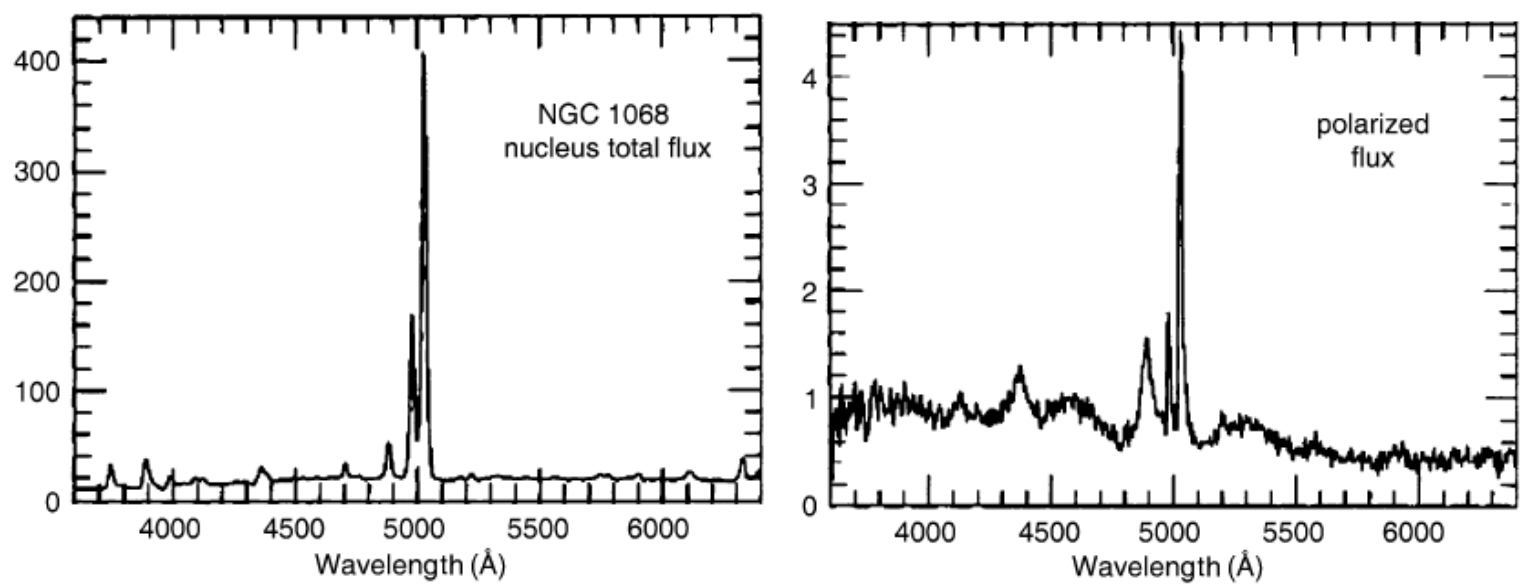

Figura 1.26: espectro da galáxia Seyfert NGC 1068. O painel da esquerda mostra o fluxo total e suas linhas de emissão estreitas. Com a luz palarizada (painel da direita), linhas largas de emissão (como $\mathrm{H} \beta$ e $\mathrm{H} \gamma$ ) típicas de Seyferts 1 são visíveis. Fonte: Miller et al. (1991).

A interpretação desta observação (figura 1.27) é que a NGC 1068 possui uma BLR, mas a observação direta dela é obscurecida por algum material absorvente. Entretanto, este material não cobre a BLR em todas as direções. Podemos concluir que a diferença entre Seyferts 1 e 2 se origina da orientação do disco de acreção (e então do material absorsor) em relação à linha de visada.

Da razão entre a abundância de Seyfert 1 e Seyfert 2 (que é cerca de 1:2), a fração de ângulo sólido na qual a BLR é obscurecida pode ser estimada. Esta razão nos diz que aproximadamente $2 / 3$ do ângulo sólido em torno do SMBH é coberto por material absorsor. O estudo desta estrutura obscurecedora (entre 0,1 10 pc a partir do SMBH) levou, entre outras coisas, ao melhor entendimento da dicotomia entre estes dois tipos de AGN. A principal hipótese (Netzer, 2013) é que esta região seja formada por uma estrutura opticamente espessa, composta de poeira e gás molecular no formato de um toro, com uma pequena abertura central e uma dimensão externa muito maior.

\subsection{Emissão difusa}

\section{Via Láctea e galáxias próximas}

Observações em gama ao longo do disco e em direção ao centro da Via Láctea destacam uma faixa de emissão difusa (figura 1.28) que, acima de $\sim 100 \mathrm{MeV}$, é originada principalmente por bremsstrahlung, produção e decaimento de píons, e espalhamento Compton 


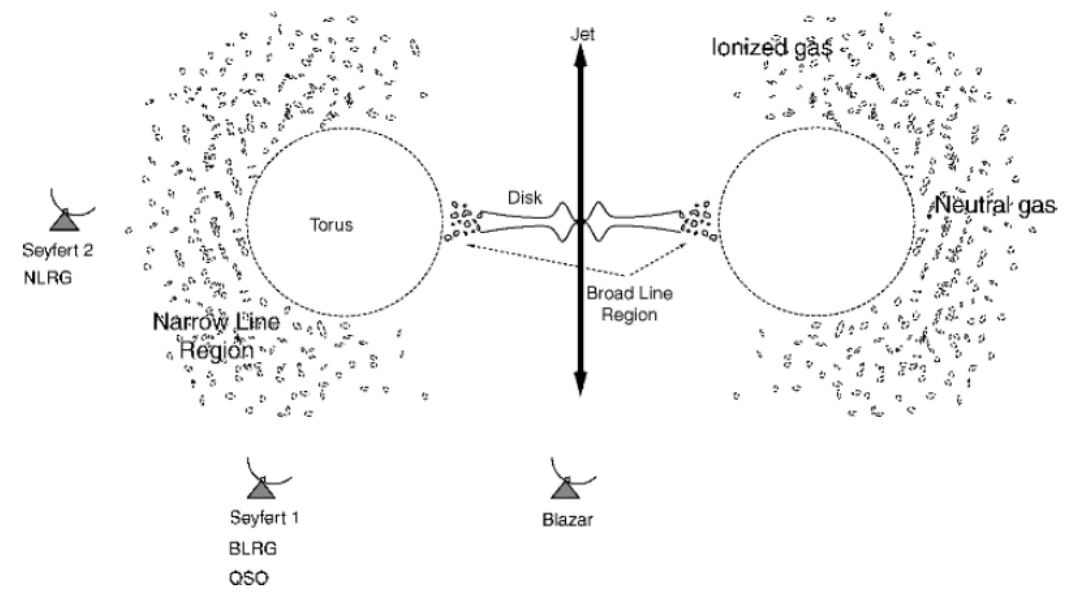

Figura 1.27: modelo da unificação dos AGNs. O disco de acreção é cercado por um toro espesso contendo poeira que obscurece o centro do AGN. Quando observado por uma direção próxima ao plano do disco, a observação direta da BLR é bloqueada, e tudo o que detectamos é a NLR. Se observado por um ângulo próximo ao eixo de simetria, tanto a BLR quanto a NLR aparecem. Se o jato for observado diretamente, o AGN aparece como um blazar. Fonte: Schneider (2014).

inverso. Sua investigação nos dá pistas sobre a origem e transporte de raios cósmicos na Via Láctea e em algumas galáxias vizinhas.

Antes do Fermi entrar em operação, a única galáxia próxima detectada como uma fonte de raios $\gamma$ era a Grande Nuvem de Magalhães (LMC), observada pelo Compton Gamma-ray Observatory como uma fonte extensa com um fluxo de raios $\gamma$ compatível com uma população de raios cósmicos similar à da Via Láctea (Massaro et al., 2016).

Com as observações do Fermi-LAT, a Pequena Nuvem de Magalhães (SMC) e a galáxia de Andrômeda entraram para a lista como duas novas fontes extensas (Massaro et al., 2016). O fluxo de raios $\gamma$ observado foi usado para calcular um limite superior para a densidade média de raios cósmicos na SMC de $\sim 15 \%$ do valor medido localmente na Via Láctea (Massaro et al., 2016). Contudo, pulsares na SMC podem contribuir com uma fração substancial do fluxo em raios $\gamma$, fazendo com que sua densidade de raios cósmicos seja ainda menor. Andrômeda mostra um fluxo de raios $\gamma$ que equivale à metade do fluxo da Via Láctea, implicando, mais uma vez, numa menor densidade de raios cósmicos (Massaro et al., 2016).

Em todas estas galáxias inativas, a densidade de raios cósmicos inferida a partir do fluxo em raios $\gamma$ é aproximadamente proporcional à taxa de formação estelar estimada, suportando a ideia de que raios cósmicos são acelerados em regiões de alta formação estelar como resultado da grande quantidade de energia cinética depositada por ventos estelares e explosões de supernova no ISM (Massaro et al., 2016). 


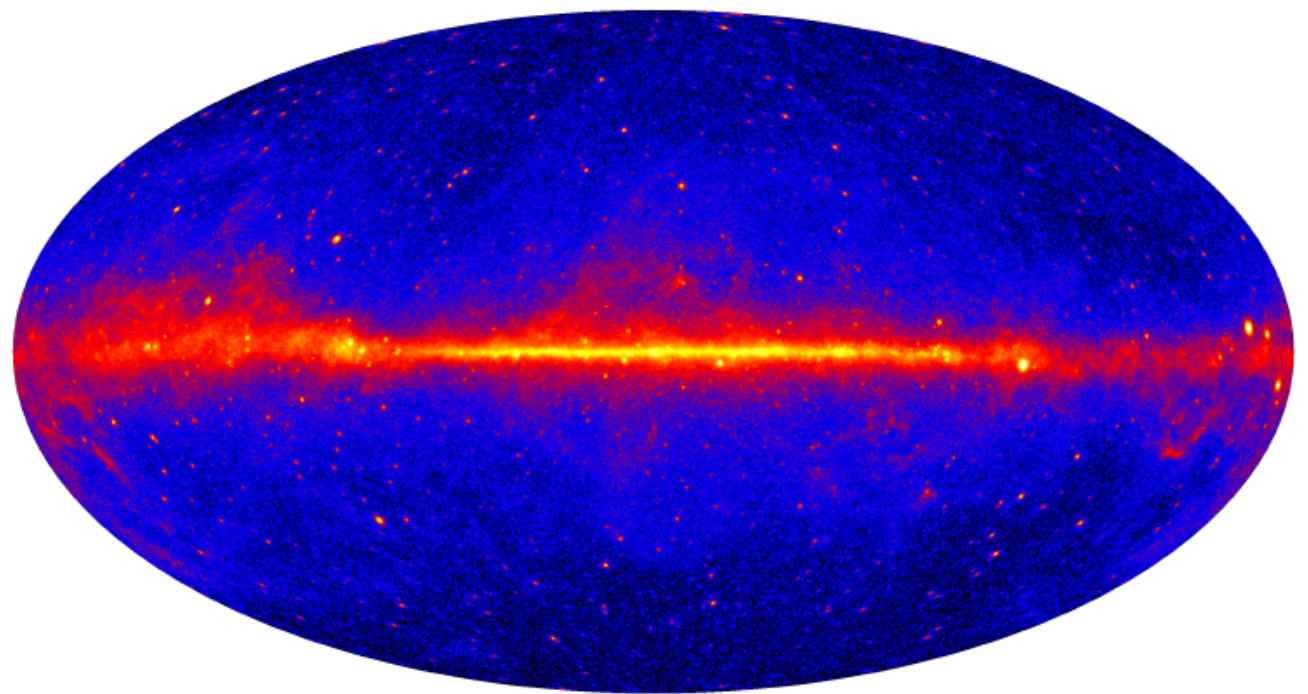

Figura 1.28: céu em raios $\gamma$ observado pelo Fermi após 60 meses de operação em energias acima de $1 \mathrm{GeV}$. A componente difusa da Via Láctea é facilmente identificada. A maioria das fontes pontuais são blazares e pulsares. Fonte: NASA/Fermi-LAT Collaboration.

\section{Componente extragaláctica}

Quando todas as fontes e emissão difusa da Via Láctea são subtraídas do Fermi allsky map, ainda resta um fluxo praticamente isotrópico chamado de extragalactic $\gamma$-ray background (EGB). Uma análise recente (Ackermann et al., 2015a) mostra o espectro do EGB entre 0,1 e $820 \mathrm{GeV}$ sendo bem representado por uma lei de potência com um corte exponencial acima de $\sim 300 \mathrm{GeV}$. O corte em altas energias é consistente com o efeito da atenuação pela extragalactic background light (EBL - subseção 1.3.8). Apesar do formato do espectro ser consistente, a normalização do EGB varia em $\pm 15 \%$ dependendo dos detalhes do modelo galáctico de foreground.

Ainda não se sabe se todo o EGB é resultado de fontes não resolvidas ou de algum processo difuso real, como a possível aniquilação de matéria escura. A figura 1.29 mostra a análise feita por Ajello et al. (2015a), incluindo o espectro do EGB, mais a contribuição de várias classes de AGNs.

\subsection{Horizonte cósmico de raios $\gamma$}

Fótons de alta energia (very high energy - VHE; $30 \mathrm{GeV}$ - $30 \mathrm{TeV}$ ) não viajam desimpedidos através de distâncias cosmológicas. Uma atenuação no fluxo é esperada devido à produção de pares por interações fóton-fóton com os fótons de baixa energia da extragalactic background light (EBL - a radiação emitida por estrelas e AGNs que permeia o Universo) em UV, óptico e infravermelho (Ackermann et al., 2016).

Uma característica interessante no espectro em VHE de fontes extragalácticas, como consequência da absorção pela EBL, é o cosmic gamma-ray horizon (CGRH). O CGRH 


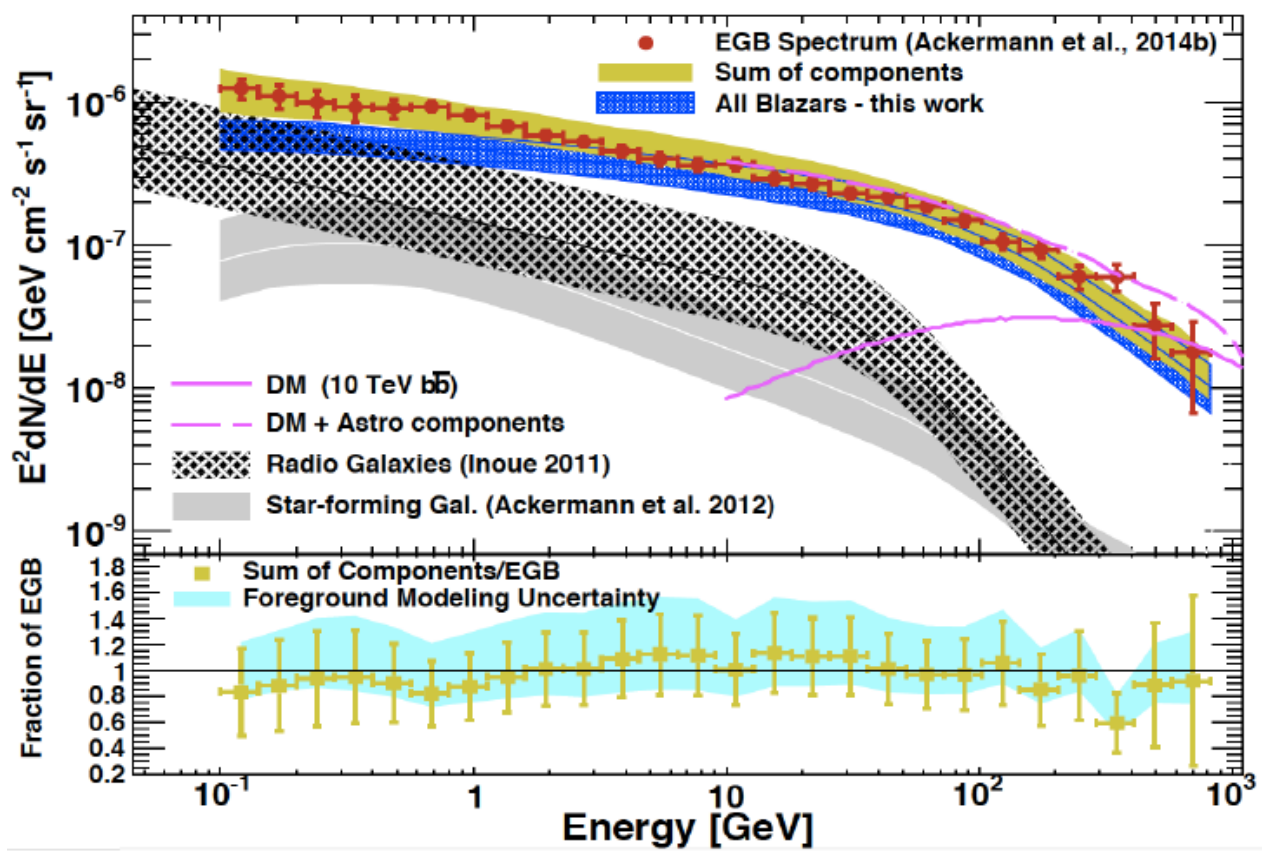

Figura 1.29: contribuição em raios $\gamma$ para o EGB de blazares, star-forming galaxies, e rádio-galáxias, comparadas à intensidade medida do EGB. Um hipotético sinal induzido por aniquilação de matéria escura é representado pela linha rosa contínua. A assinatura da absorção pela EBL é visível como um corte em altas energias. Fonte: Ajello et al. (2015b).

é, por definição (Ackermann et al. 2016), a energia $E_{0}$ na qual a profundidade óptica da produção de pares por interações fóton-fóton torna-se unitária $\left(\left.\tau(z)\right|_{E_{0}}=1\right)$ em função do redshift. Em outras palavras, dados o redshift da fonte e a energia do seu fóton mais energético, a profundidade óptica pode ser calculada.

Porém, a profundidade óptica pode ser estimada ajustando-se modelos de síncrotron/SSC à SED de algumas fontes e comparando-os com os dados. Este método, descrito em detalhes em Domínguez et al. (2013), permite a derivação de limites superiores para o redshift de vários AGNs do Fermi (como os BL Lacs, cuja dificuldade de realizar medidas baseadas em seu espectro é bastante alta).

Devido ao comportamento exponencial da atenuação do fluxo $\left(I_{\text {observado }}=I_{0} e^{-\tau}\right)$, uma definição alternativa do CGRH é a energia na qual o espectro intrínseco é atenuado pela EBL por um fator de 1/e (Domínguez et al., 2013). A figura 1.30 deixa evidente a atenuação dos fótons VHE com o redshift.

\subsection{Motivação e objetivos}

No Universo local $(z \approx 0)$, a maior parte da atividade em núcleos de galáxias é observada em Low-luminosity AGNs (LLAGNs). Estes objetos possuem um SMBH com baixos níveis de atividade em seu centro, colocando-os numa posição entre galáxias ina- 


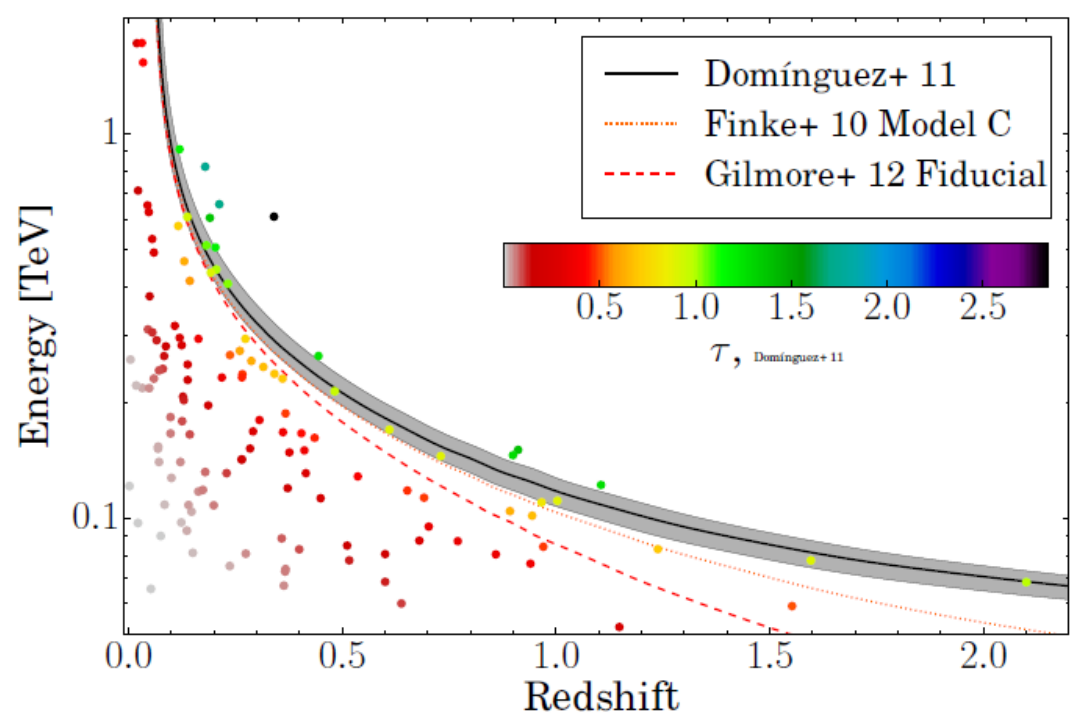

Figura 1.30: fóton de maior energia versus redshift da fonte com base no segundo catálogo de hard sources do Fermi (Ackermann et al., 2016). Os símbolos são codificados com cores baseadas na profundidade óptica, $\tau$, estimada do modelo de EBL disponível em Domínguez et al. (2013). Diferentes estimativas do CGRH são plotadas baseadas nos modelos de EBL de Finke et al. (2010), representado pela linha laranja pontilhada, Domínguez et al. (2013), representado pela linha preta sólida, com sua incerteza em cinza, e Gilmore et al. (2012), representado pela linha vermelha pontilhada. O CGRH ainda nos dá a oportunidade de estimar o redshift da fonte, baseado na detecção de seu fóton mais energético.

tivas e AGNs mais tradicionais, de maior luminosidade, tais como quasares e Seyferts. Embora observados em grande número, LLAGNs ainda são pouco compreendidos, com várias questões em aberto sobre a física do SMBH, sua acreção e outflows (Ho, 2009; Nemmen et al., 2014). Pouco se sabe sobre a origem da emissão em raios $\gamma$ (quando há) desta população.

Com base em 7 anos de dados do telescópio espacial Fermi, montamos um survey de variabilidade em raios $\gamma$ para os LLAGNs mais brilhantes do Universo local. As curvas de luz, geradas com a metodologia descrita no capítulo 2, nos dão a chance de explorar fenômenos de alta energia em torno de buracos negros de baixa atividade, assim como inferir limites superiores para o tamanho de suas regiões emissoras. Este trabalho consiste da caracterização - busca por flares e periodicidade - destas curvas de luz.

A única fonte extragaláctica (quasi-)periódica em raios $\gamma$ conhecida até então é o BL Lac PG1553 + 113, cuja análise e possíveis interpretações foram publicadas recentemente Ackermann et al., 2015b). A busca por periodicidade em outras fontes é de essencial importância para uma melhor interpretação do fenômeno. Nosso survey exploratório apresenta mais um AGN candidato a fonte (quasi-)periódica. À parte das periodicidades e curvas de luz, a descoberta fortuita de 3 novas fontes de raios $\gamma$ é reportada no capítulo 3. 
CAPÍTULO 2

\section{Explorando os dados do Fermi}

Vários fatores comprometem a qualidade dos dados brutos observados pelo Fermi. A emissão de raios $\gamma$ pelo limbo terrestre, devido principalmente à interação de raios cósmicos com partículas da alta atmosfera, e o período de tempo em que o telescópio orbita a região da anomalia magnética do Atlântico Sul (figura 2.1) são os primeiros cuidados que devem ser tomados.

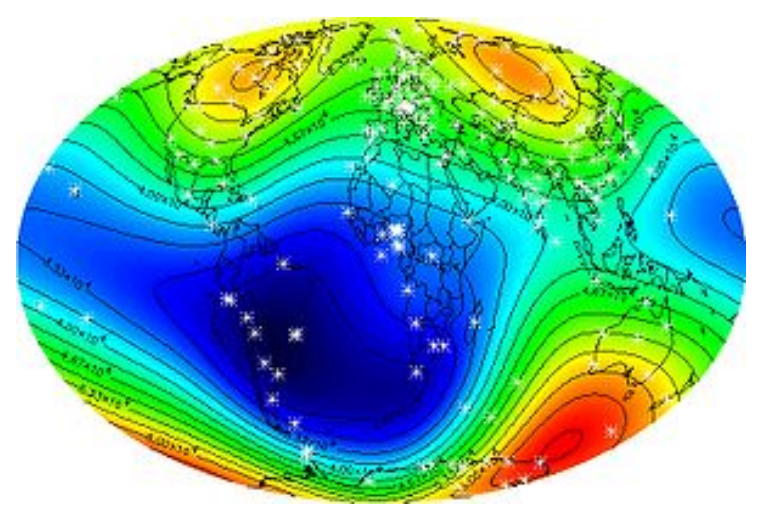

Figura 2.1: anomalia magnética do Atlântico Sul. Região em azul onde a parte mais interna do cinturão de Van Allen tem máxima aproximação com a superfície da Terra, prejudicando o funcionamento de satélites de órbita baixa. Fonte: domínio público. Retirada de https://pt.wikipedia.org/wiki/Anomalia_do_Atlantico_Sul.

A resposta dos instrumentos do LAT é dada em função do ângulo de inclinação (ângulo entre a direção da fonte e a normal do LAT). O número de contagens detectado de uma fonte depende da quantidade de tempo que a fonte ficou sob um dado ângulo de inclinação durante a observação. Um livetime é definido como o tempo em que o LAT observou uma dada posição do céu em um dado ângulo de inclinação. O array desses livetimes em todos os pontos do céu é chamado de livetime cube. 
Os mapas de exposição gerados a partir do livetime cube diferem significativamente da noção usual de mapas de exposição, que são essencialmente integrais da área efetiva sobre o tempo. O cálculo de exposição usado consiste de uma integral da resposta total do equipamento, com base no livetime cube, sobre a região de interesse 1 (ROI - daqui em diante, do inglês region of interest. Região com $15^{\circ} \sim 30^{\circ}$ de raio centrada no alvo).

\section{Fermi Science Tools}

Para a redução destes dados brutos, o Fermi Science Support Center (FSSC) disponibiliza um conjunto de ferramentas computacionais chamadas de Fermi Science Tools, assim como seu tutorial 2 . Algumas análises, como a produção de curvas de luz, tornamse bastante ineficientes com o uso direto destas ferramentas, podendo demorar dias ou semanas, além de extrair a informação de maneira incompleta.

Um código mais eficiente para a criação de curvas de luz, baseado nas Fermi Science Tools, v10r0p5 3 , foi desenvolvido e testado neste projeto (seção 2.2).

Neste capítulo elucidaremos a metodologia utilizada, descrevendo o passo a passo do nosso código e compararemos seus resultados com resultados já conhecidos na literatura. Ainda explicitaremos, de maneira informal, a base teórica por trás da likelihood analysis e dos métodos de análise de séries temporais.

\subsection{Metodologia}

As curvas de luz em raios $\gamma$ podem ser criadas tanto por fotometria de abertura quanto por uma likelihood analysis. A likelihood analysis é mais rigorosa e permite que aproveitemos ao máximo a sensibilidade do Fermi. Ela também permite medidas mais acuradas do fluxo, já que o background pode ser modelado e modelos mais detalhados de fontes podem ser usados. Entretanto, a fotometria de abertura também pode ser útil - ela nos fornece uma medida do fluxo independente do modelo e tem menor custo computacional. Na montagem das curvas de luz e SEDs dos nossos alvos, a likelihood analysis foi o principal método utilizado - só recorremos à fotometria de abertura quando suspeitamos que a fonte fosse periódica, onde uma análise temporal de alta resolução era necessária. A likelihood analysis usa o método de maximum likelihood estimation (MLE), descrito brevemente na seção 2.1.1, que consiste em estimar os melhores parâmetros para o espectro da fonte com base nos dados e em um modelo de entrada.

\footnotetext{
${ }^{1}$ Fermi-LAT Collaboration - Livetime and Exposure: http://fermi.gsfc.nasa.gov/ssc/data/ analysis/documentation/Cicerone/Cicerone_Data_Exploration/livetime_and_exposure.html

${ }^{2}$ Fermi-LAT Collaboration - Analysis Threads: http://fermi.gsfc.nasa.gov/ssc/data/analysis/ scitools/

${ }^{3}$ Fermi-LAT Collaboration - Installing the Fermi Science Tools: http://fermi.gsfc.nasa.gov/ssc/ data/analysis/software/
} 
A determinação do fluxo e modelagem espectral das fontes através do método de likelihood analysis é feita de duas maneiras distintas, de acordo com a situação estudada: a unbinned likelihood é a mais utilizada para análise de séries temporais dos dados do LAT, onde o número de eventos em cada bin de tempo é esperado ser pequeno. Entretanto, para bins abrangendo intervalos temporais muito grandes, análises que incluem fontes de fundo muito fortes (como o plano da Via Láctea) e longos períodos de exposição (da ordem de anos), a binned likelihood é recomendada.

O modelo de entrada que usamos na likelihood é dado por um catálogo de fontes de raios $\gamma$ (3FGL - com informações sobre a posição e espectro) do próprio LAT (Acero et al. 2015a) 4. Este modelo é importado pelas Fermi Science Tools através de um arquivo xml (figura 2.2) e comparado com os dados já reduzidos. Após o processo de likelihood, obtemos um modelo de saída (arquivo xml de saída), que usamos na montagem de um model map (figura 2.3) ou TS map (subseção 2.1.2).

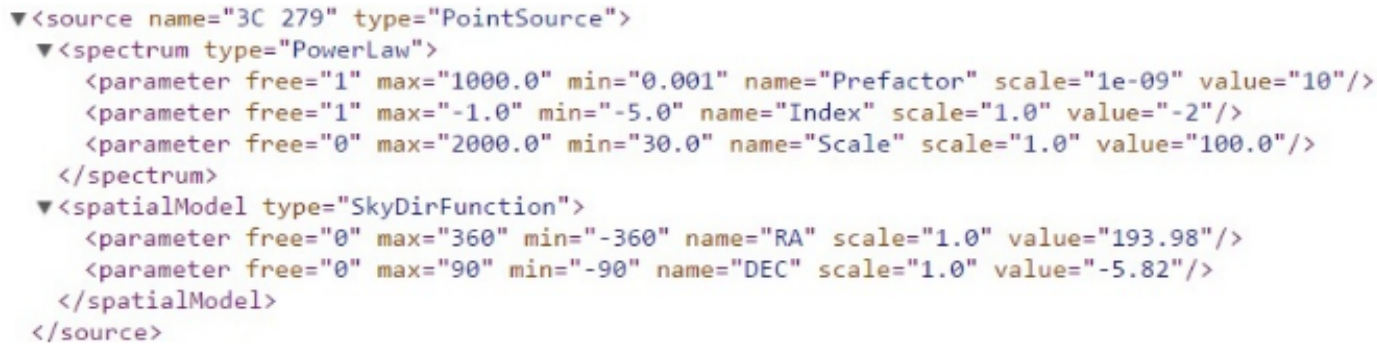

Figura 2.2: parte do modelo xml de entrada, com informações sobre a posição (RA e DEC) e espectro (Prefactor, Index e Scale) do blazar 3C 279. O blazar foi modelado de acordo com a lei de potência $\frac{d N}{d E}=N_{0}\left(\frac{E}{E_{0}}\right)^{-\gamma}$, em unidades de $\mathrm{cm}^{-2} \mathrm{~s}^{-1} \mathrm{MeV}^{-1}$ (mais detalhes na seção 2.1.1).

\subsubsection{Maximum likelihood estimation}

O algoritmo usado nas análises ajusta os parâmetros do modelo (inserido com o arquivo xml) de acordo com os dados obtidos pelo telescópio através do método de MLE. Neste método, os parâmetros são estimados de forma que a likelihood, L, de obtenção dos dados observados seja maximizada.

Os dados do Fermi-LAT, para um determinado intervalo de tempo, são divididos em bins espaciais e de energia, e cada bin contém um pequeno número de contagens caracterizado pela distribuição de Poisson 5 . A likelihood $L$ é o produto das probabilidades de observarmos as contagens detectadas em cada bin. Assumindo que o número esperado de contagens no i-ésimo bin é $m_{i}(\boldsymbol{\theta})$ (onde $\boldsymbol{\theta}$ é um conjunto de parâmetros a ser ajustado),

\footnotetext{
${ }^{4}$ Catálogo também disponível online em http://www.asdc.asi.it/fermi3fgl/. Acesso em 05-072016.

${ }^{5}$ Mais detalhes em http://fermi.gsfc.nasa.gov/ssc/data/analysis/documentation/Cicerone/ Cicerone_Likelihood/Likelihood_formula.html. Acesso em 05-07-2016.
} 

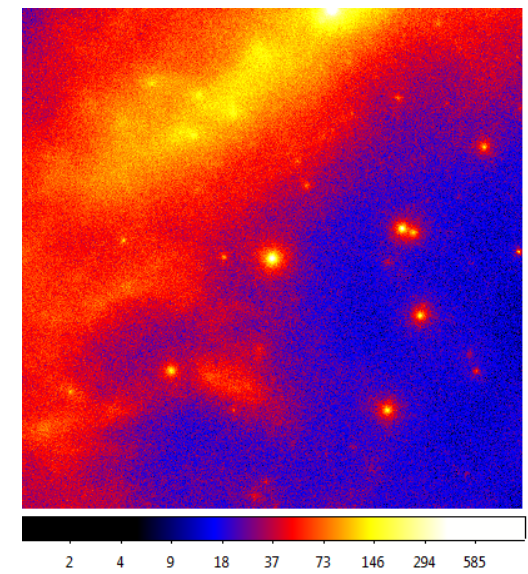

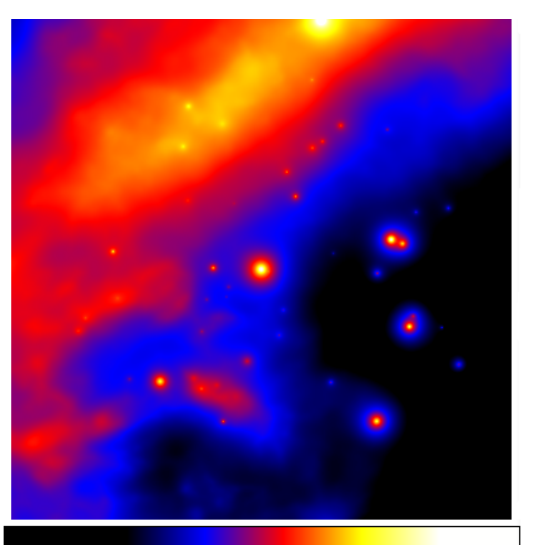

$\begin{array}{lllllllll}15 & 18 & 22 & 32 & 52 & 90 & 167 & 321 & 627\end{array}$

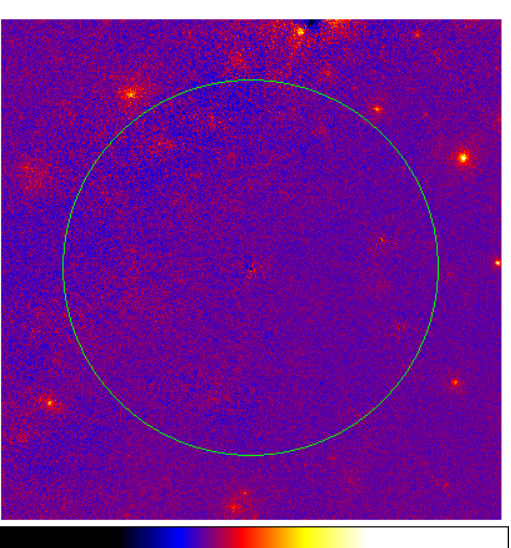

$-93$

28

Figura 2.3: esquerda: mapa de contagens observado, centrado na Seyfert NGC 1275, já reduzido com as Fermi Science Tools. Centro: model map montado a partir do arquivo xml de saída da binned likelihood (é basicamente um mapa de contagens modelado com infinitos eventos ocorridos). Direita: mapa de resíduos obtido pela subtração (mapa de contagens)-(model map). Além de um raio de $15^{\circ}$, delimitado pelo círculo verde, apenas as fontes muito fortes foram modeladas. A região inteira possui $20^{\circ} \times 20^{\circ}$.

a probabilidade de detectarmos $n_{i}$ contagens neste bin é $P_{i}=\frac{m_{i}^{n_{i}} e^{-m_{i}}}{n_{i} !}$. A likelihood $L$ é, então, o produto de todos os $P_{i}$ :

$$
L \equiv \prod_{i=1}^{N} P_{i}\left(n_{i} \mid m_{i}(\boldsymbol{\theta})\right) .
$$

A equação acima é a base da likelihood analysis. Para encontrar os parâmetros que maximizam a likelihood, simplesmente derivamos $L$ em relação ao parâmetro desejado e igualamos a zero:

$$
\frac{\partial L}{\partial \theta_{i}}=0
$$

As fontes estudadas foram modeladas de acordo com a lei de potência

$$
\frac{d N}{d E}=N_{0}\left(\frac{E}{E_{0}}\right)^{-\gamma}
$$

em contagens $\times \mathrm{cm}^{-2} \mathrm{~s}^{-1} \mathrm{MeV}^{-1}$, onde, como de costume no estudo de AGNs (Abdo et al., 2010b), esperamos que a contagem de fótons decresça para altas energias. O parâmentro de normalização (prefactor), $N_{0}$, que representa o fluxo de fótons por intervalo de energia, e o índice espectral (Index), $\gamma$, são estimados via MLE. O parâmetro de escala, $E_{0}(S c a l e)$, possui valor fixo, determinado arbitrariamente, e $E$ é a energia dos fótons medida pelo Fermi-LAT. A figura 2.2 mostra como este modelo é importado pelas Fermi Science Tools. 


\subsubsection{Mapas de teste estatístico}

As Fermi Science Tools permitem a criação de um mapa de teste estatístico (mapa de TS) baseado numa razão de likelihoods. O TS é definido como:

$$
T S=\ln \left(\frac{L_{1}}{L_{0}}\right)^{2}
$$

onde $\frac{L_{1}}{L_{0}}$ representa a likelihood entre modelos com e sem uma fonte pontual na posição de interesse 6 . Em um mapa de TS, este cálculo é feito para determinar a significância do sinal em raios gama em cada pixel do céu. A significância estatística do sinal é dada aproximadamente pela raiz quadrada do TS. Fontes com $T S<25$ possuem uma significância estatística menor que $5 \sigma(\sqrt{T S})$ e não são inclusas nos catálogos do Fermi (1FGL, 2FGL e 3FGL) (Abdo et al. 2010a).

Um mapa de TS da região centrada no PG1553+113, com dados obtidos entre 06/2011 e 06/2013, é mostrado na figura 2.4a, onde notamos a presença de várias outras fontes com alta significância estatística. A figura 2.4b mostra a mesma região, só que agora subtraída das fontes inclusas no nosso modelo xml. Temos, portanto, um mapa de TS de resíduos, que é especialmente importante na análise de fontes fracas que sequer foram catalogadas. Ainda notamos na figura $2.4 \mathrm{~b}$ algumas poucas fontes com $T S>25$, que podem ser, por exemplo, AGNs que entraram em período de alta atividade após o lançamento do último catálogo de fontes do Fermi (3FGL, que inclui fontes com TS > 25 até agosto de 2012 (Acero et al., 2015a)).

\subsection{Pipeline de produção de curvas de luz}

Desenvolvemos o código Kame Hame $\mathrm{Ha}$ (KHH) para gerar as curvas de luz. O código foi totalmente desenvolvido em Python 7 e faz uso de várias bibliotecas voltadas para análise de dados $8^{9} b^{10}{ }^{11}$, além de importar as Fermi Science Tools. Suas principais tarefas são:

- Redução de dados.

- Análise estatística das fontes no campo (binned likelihood), que inclui o cálculo da significância estatística, fluxo e espectro para cada fonte na ROI.

\footnotetext{
${ }^{6}$ Mais detalhes em http://fermi.gsfc.nasa.gov/ssc/data/analysis/documentation/Cicerone/ Cicerone_Likelihood/Likelihood_overview.html

'Python: https://www.python.org/. Acesso em 05-07-2016.

${ }^{8}$ NumPy: http://www.numpy.org/. Acesso em 05-07-2016.

${ }^{9}$ SciPy: https://www.scipy.org/. Acesso em 05-07-2016.

${ }^{10}$ Matplotlib: http://matplotlib.org/. Acesso em 05-07-2016.

${ }^{11} \mathrm{ObsPy}$ : https://github.com/obspy/obspy/wiki. Acesso em 05-07-2016.
} 


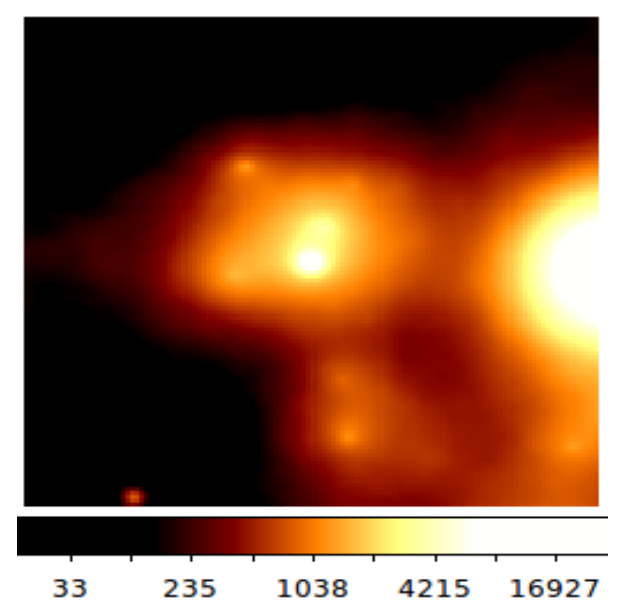

(a) mapa de TS das fontes.

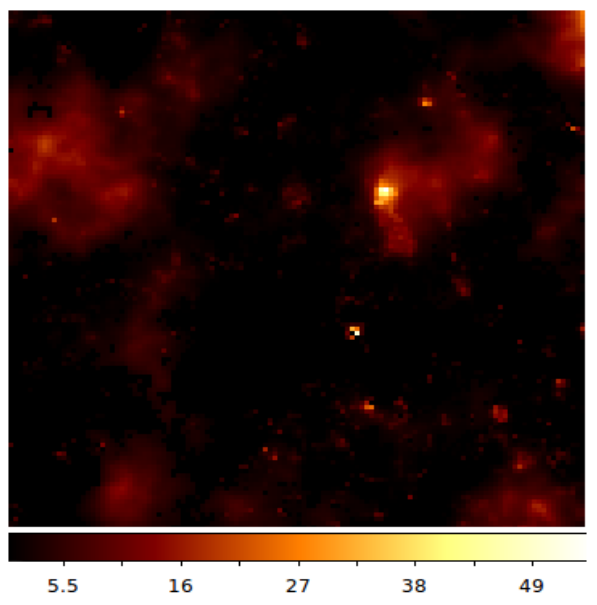

(b) mapa de TS de resíduos.

Figura 2.4: mapas de TS com $0,2^{\circ}$ por pixel envolvendo uma região de $24^{\circ} \times 24^{\circ}$ centrada no PG1553+113. A barra de cores representa aproximadamente o quadrado da significância estatística de cada fonte (significância $\approx \sqrt{T S}$ ). No cálculo destes mapas foram utilizados dois anos (06/2011 até 06/2013) de dados do Fermi-LAT num intervalo de energias entre $100 \mathrm{MeV}$ e $300 \mathrm{GeV}$.

- Construção dos mapas de TS (na maioria dos casos apenas o TS de resíduos é necessário).

- Produção de curvas de luz, com bins temporais predeterminados, para a fonte-alvo.

- Análise de Fourier, Lomb-Scargle e wavelets das curvas de luz geradas no passo anterior.

- Estimativa, caso seja necessário, de limites superiores para o fluxo em raios $\gamma$ do alvo.

Várias tarefas da pipeline foram paralelizadas e a produção de curvas de luz, em especial, chega a ser três vezes mais rápida quando executada em quatro núcleos (durando $25 \sim 30$ horas, para bins de tempo mensais em sete anos de dados).

\subsubsection{Testes da pipeline}

Antes de ser aplicado aos alvos deste projeto, o código foi testado nos blazares 3C 279 e PG 1553+113, cujas curvas de luz foram bem caracterizadas na literatura (Ackermann et al. 2015b; Paliya, 2015). Os resultados que obtivemos com o KHH reproduziram bem as curvas de luz previamente obtidas na literatura e são apresentados nos tópicos seguintes.

\section{Flare no 3C 279}

Um flare excepcional em raios $\gamma$ no blazar 3C 279 foi observado pelo Fermi-LAT em junho de 2015. Na análise em questão, foi utilizada uma ROI de $15^{\circ}$ de raio centrada 
no 3 C 279, com dados num intervalo de tempo de 16 dias em torno do período de alta atividade. Os dados foram divididos em bins de 1 dia e envolvem energias entre 0.1 e 300

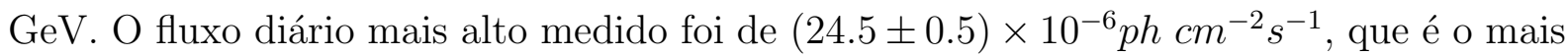
alto já detectado para o 3C 279 (Paliya, 2015). A figura 2.5 compara e mostra que nosso resultado reproduziu bem a curva de luz já conhecida na literatura.
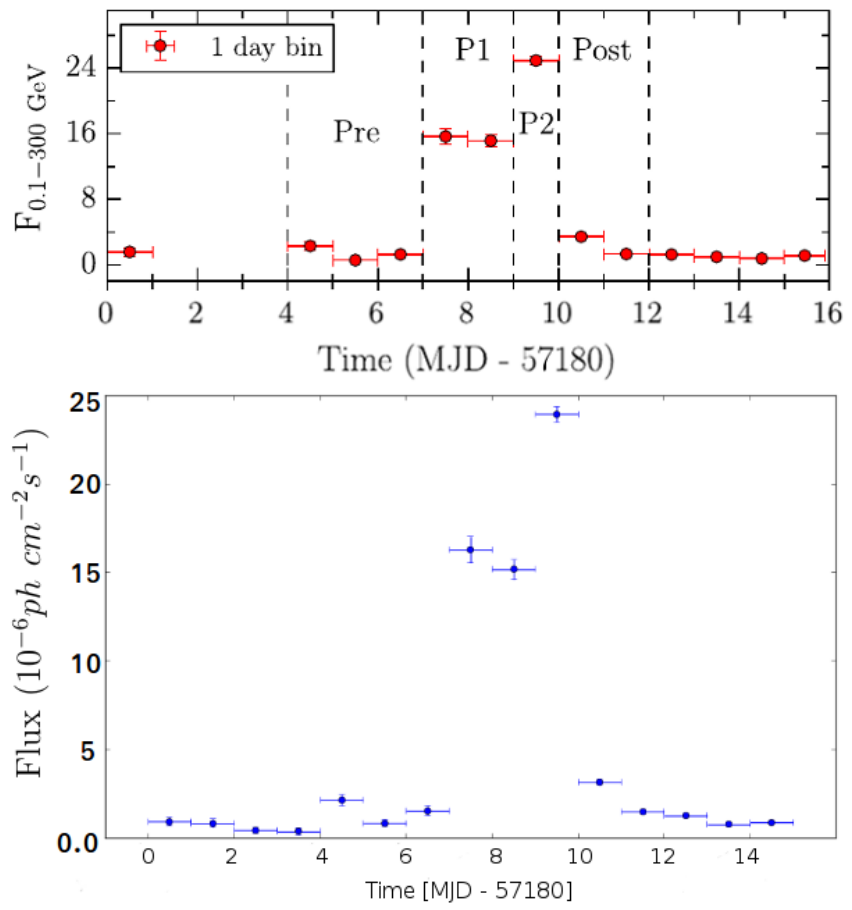

Figura 2.5: curva de luz com bins diários do 3C 279 abrangendo um período de flare em junho de 2015 (entre 07/06/2015, às 00:00:00, e 23/06/2015, às 00:00:00 - Escala em Modified Julian Day - MJD). Acima: resultado de Paliya (2015), com fluxo em unidades

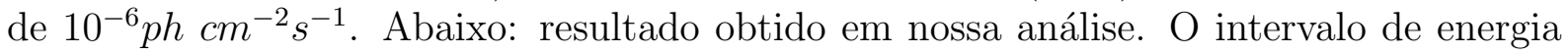
adotado nas duas análises foi entre $100 \mathrm{MeV}$ e $300 \mathrm{GeV}$.

Os dados utilizados nas duas análises, porém, são sutilmente diferentes. Nosso código usa a versão Pass 8 dos dados do Fermi, que conta com um reprocessamento total dos dados de toda a missão do telescópio, incluindo melhor reconstrução de eventos, maior intervalo de energia, melhor precisão na medida da energia dos fótons e um significante aumento na área efetiva ${ }^{12}$, enquanto que os dados adotados na referência Paliya (2015) são da versão Pass \%, com menor qualidade.

\section{Periodicidade no PG $1553+113$}

Baseado nos dados do Fermi-LAT, uma quasi-periodicidade foi, pela primeira vez, identificada em raios $\gamma$ num AGN. O ciclo observado de $2.18 \pm 0.08$ anos do BL Lac PG

\footnotetext{
${ }^{12}$ Usando a versão Pass 8 dos dados: http://fermi.gsfc.nasa.gov/ssc/data/analysis/ documentation/Pass8_usage.html. Acesso em 06-07-2016.
} 
$1553+113$, representado pelos três picos na figura 2.7, é coerente com oscilações observadas para o blazar no rádio e no óptico (Ackermann et al., 2015b).

O processo de criação de curvas de luz estima, através do método de MLE, o Prefactor, $N_{0}$ (em unidades de $p h / M e V / \mathrm{cm}^{2} / s$ ), e o Index, $\gamma$, para o alvo e para as fontes próximas que estão livres (ver próximo tópico). Quando o Index não apresenta variações bruscas ao decorrer do tempo (figura 2.6), podemos fixá-lo e tentar um novo fit. A curva de luz da figura 2.7 foi gerada com o Index fixo em $\gamma=1.6$.

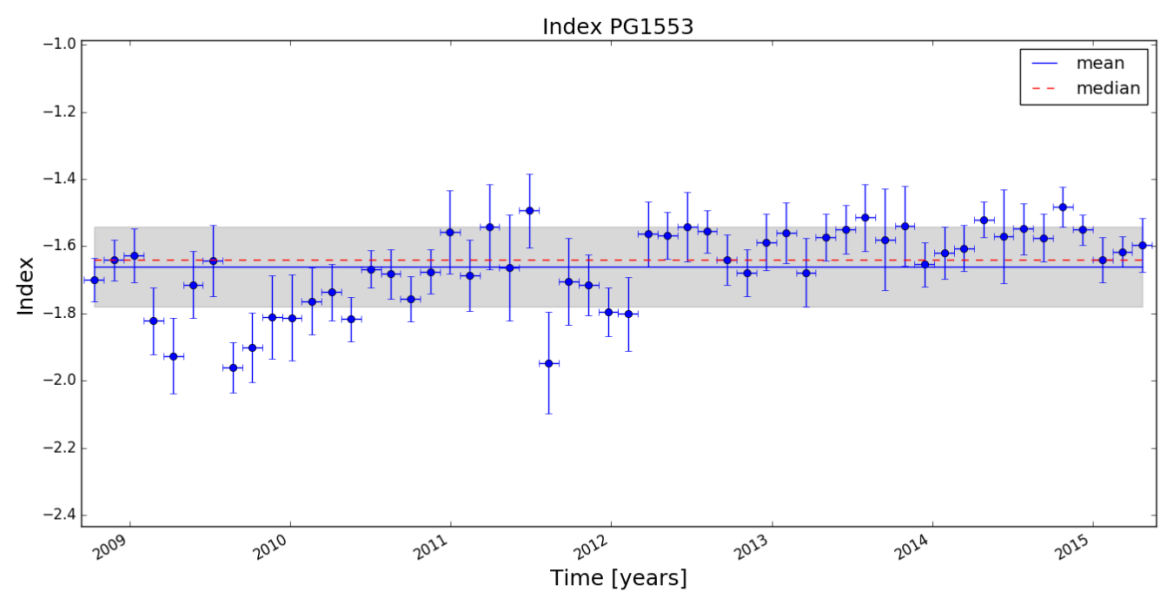

Figura 2.6: Index em escala negativa em função do tempo. A área hachurada representa o desvio padrão das medidas.

\section{Fontes próximas}

O número de fontes com parâmetros livres na ROI é escolhido a critério do pesquisador. Quanto maior o número de fontes livres, mais precisa será a curva de luz e maior será o custo computacional.

Flares em fontes próximas podem causar um sinal espúrio no alvo, como o falso-flare no final de 2009 detectado no BL Lac GB6 J1231+1421 (figura 2.8), causado pela Messier 87 , localizada a $\sim 2^{\circ}$ dali. Este problema é ainda maior quando queremos analisar uma fonte fraca nas proximidades de uma fonte forte. A solução é deixar os parâmetros de fontes fortes nas proximidades do alvo variarem livremente na análise.

\subsection{Análise de séries temporais}

As curvas de luz foram analisadas com métodos de caracterização espectral comumente utilizados no contexto de séries temporais: fast Fourier transform (FFT), Lomb-Scargle periodogram (LSP) e continuous wavelet transform (CWT), sendo o último o mais adequado para a detecção de periodicidades transientes. 

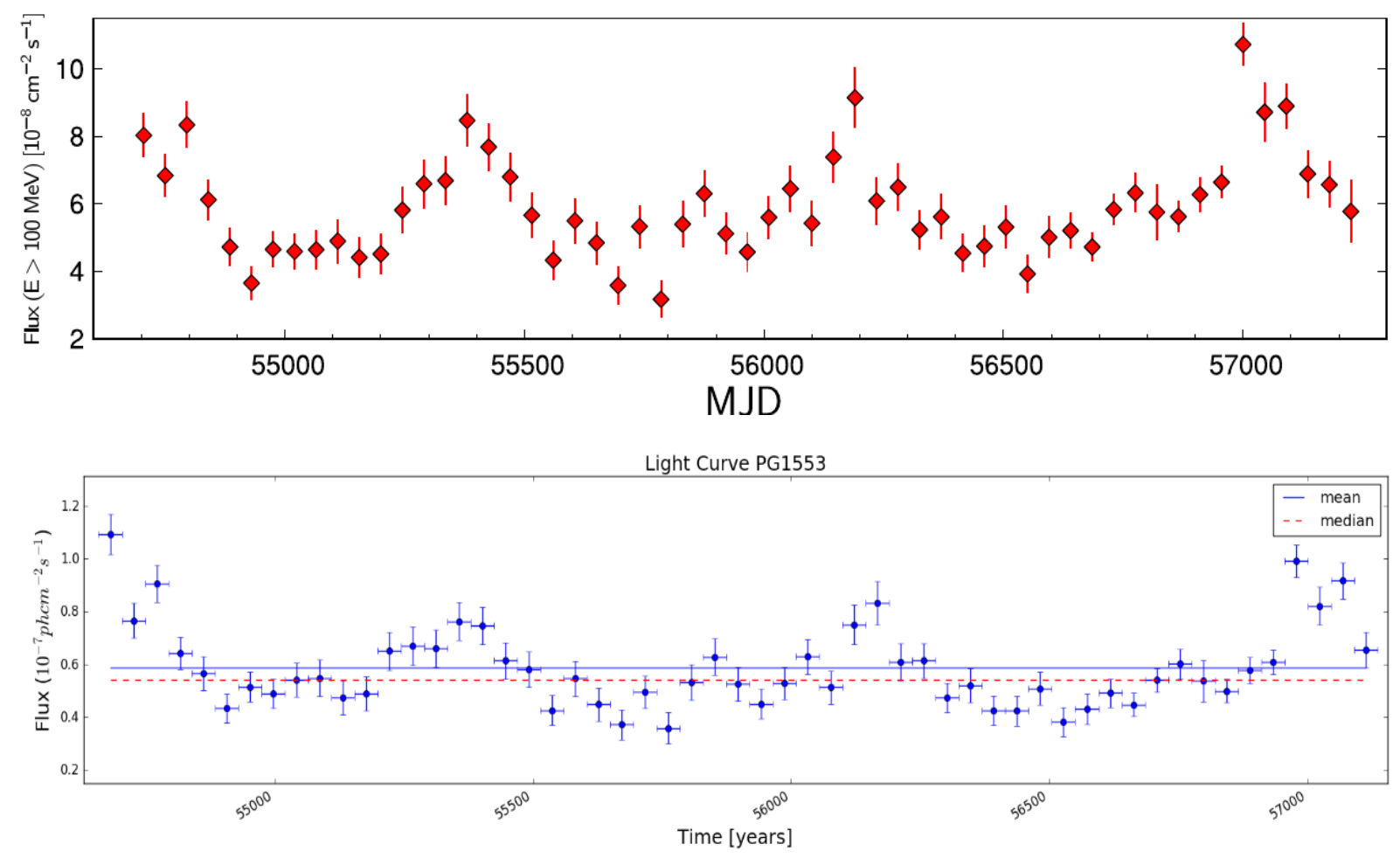

Figura 2.7: acima: curva de luz com bins de 45 dias (Ackermann et al. 2015b). Abaixo: curva de luz obtida usando os mesmos parâmetros de análise adotados por Ackermann et al. (2015b). O intervalo de energia adotado nas duas análises foi entre $100 \mathrm{MeV}$ e 300 Gev. As pequenas discrepâncias observadas podem ser justificadas pela quantidade de fontes livres na ROI.

A busca por periodicidade em fontes extragalácticas de raios $\gamma$ ganhou notável destaque após o artigo de Ackermann et al. (2015b), que sugere vários cenários para explicar o sinal quasi-periódico observado no PG 1553+113 (figura 2.7); entre eles:

- Precessão do jato do AGN (Caproni and Abraham, 2004).

- Sistema binário de SMBH, com uma massa total $\sim 10^{8} M_{\odot}$ e uma separação da ordem de miliparsecs (Lobanov, 2004).

- Instabilidades no escoamento de acreção no SMBH (Tchekhovskoy et al., 2011).

\subsubsection{Fourier e Lomb-Scargle}

As possíveis periodicidades no sinal são computadas pelo nosso código, inicialmente, através das análises de Fourier e Lomb-Scargle.

A transformada de Fourier é uma das ferramentas mais usadas para transformar funções ou sequências de dados do domínio temporal para o domínio de frequências. Qualquer função periódica e contínua pode ser representada como uma combinação linear de senos e cossenos (Shatkay, 1995). Tal função, $f(t)$, pode ser escrita como 

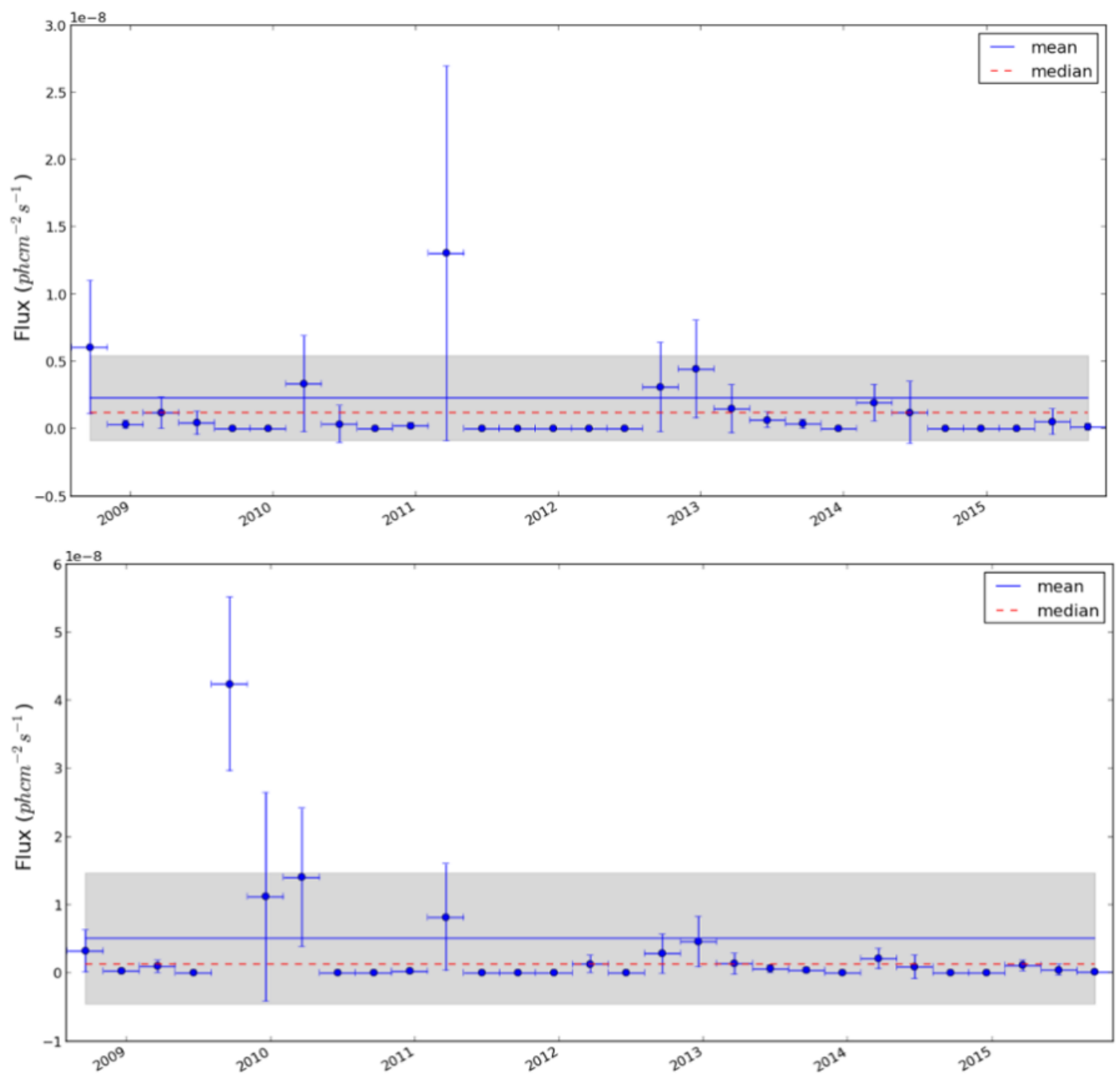

Figura 2.8: acima: falso-flare no BL Lac GB6 J1231+1421 no final de 2009. Abaixo: liberando os parâmetros da M87, fonte forte nas proximidades do alvo, o falso-flare desaparece, indicando que o sinal era espúrio. Voltaremos a falar do flare da M87 no capítulo 3.

$$
f(t)=\sum_{k=1}^{n}\left(A_{k} \cos \left(\omega_{k} t\right)+B_{k} \sin \left(\omega_{k} t\right)\right),
$$

onde $A$ e $B$ são as amplitudes de cada uma das frequências, $\omega$, que compõem o sinal.

Podemos usar a fórmula de Euler para reescrever a equação 2.2 da seguinte maneira:

$$
f(t)=\sum_{k=1}^{n}\left[\frac{A_{k}}{2}\left(e^{i \omega_{k} t}+e^{-i \omega_{k} t}\right)+\frac{B_{k}}{2 i}\left(e^{i \omega_{k} t}-e^{-i \omega_{k} t}\right)\right] .
$$

E, denotando

$$
\begin{gathered}
C_{k}=\frac{A_{k}-i B_{k}}{2} \text { para } k>0, \\
C_{k}=\frac{A_{k}+i B_{k}}{2} \text { para } k<0, \\
C_{0}=0 \mathrm{e}
\end{gathered}
$$




$$
\omega_{k}=-\omega_{-k} \text { para } K<0,
$$

podemos compactar $f(t)$ com a seguinte notação

$$
f(t)=\sum_{-n}^{n} C_{k} e^{i \omega_{k} t} .
$$

No caso de um espectro de frequências contínuo, podemos reescrever $f(t)$ como $($ Bloomfield, 2004)

$$
f(t)=\int_{-\infty}^{\infty} C(\omega) e^{i \omega t} d \omega
$$

onde $C(\omega)$ representa a amplitude relativa de um espectro contínuo de frequências. A transformada de Fourier de $f(x)$ trata exatamente de encontrarmos os valores de $C(\omega)$, dada uma função periódica $f(t)$, e é definida como (Bloomfield, 2004)

$$
C(\omega) \equiv F(\omega)=\int_{-\infty}^{\infty} f(t) e^{-i \omega t} d t
$$

A equação acima permite que encontremos a amplitude relativa de cada frequênia $\omega$ num sinal periódico, dado que a integral convirja.

O método de Lomb-Scargle é indicado para séries temporais incompletas, como aquelas onde a MLE não convergiu em alguns bins, e calcula o espectro de frequências baseado no ajuste por mínimos quadrados da função senoidal

$$
d\left(t_{i}\right)=A \cos \left(2 \pi f t_{i}-\theta\right)+B \sin \left(2 \pi f t_{i}-\theta\right)+n_{i}
$$

nos dados da nossa série temporal. Na equação, $d\left(t_{i}\right)$ representa nossa medida no tempo $t_{i}, A$ e $B$ são as amplitudes do cosseno e do seno, $f$ é a frequência a ser estimada, $n_{i}$ representa o ruído no tempo $t_{i}$ e $\theta$ é escolhido de forma que as funções seno e cosseno sejam ortogonais (Scargle, 1982).

A figura 2.9 mostra nossa análise espectral do PG 1553+113, feita para uma curva de luz com melhor resolução temporal que a mostrada na figura 2.7.

Caso um pico significativo seja detectado no LSP, é útil fazer um diagrama de fase, que nos dá informações sobre a forma, amplitude e fase do sinal. Nosso resultado para o PG1553+113, assumindo um sinal periódico com o período $P_{L S P}=779$ dias, consta na figura 2.10 .

\subsubsection{Continuous wavelet transform}

A análise de Fourier nos dá informações sobre frequências dominantes que ocorrem durante toda a extensão do sinal; esta não nos dá informação sobre as variações locais no 

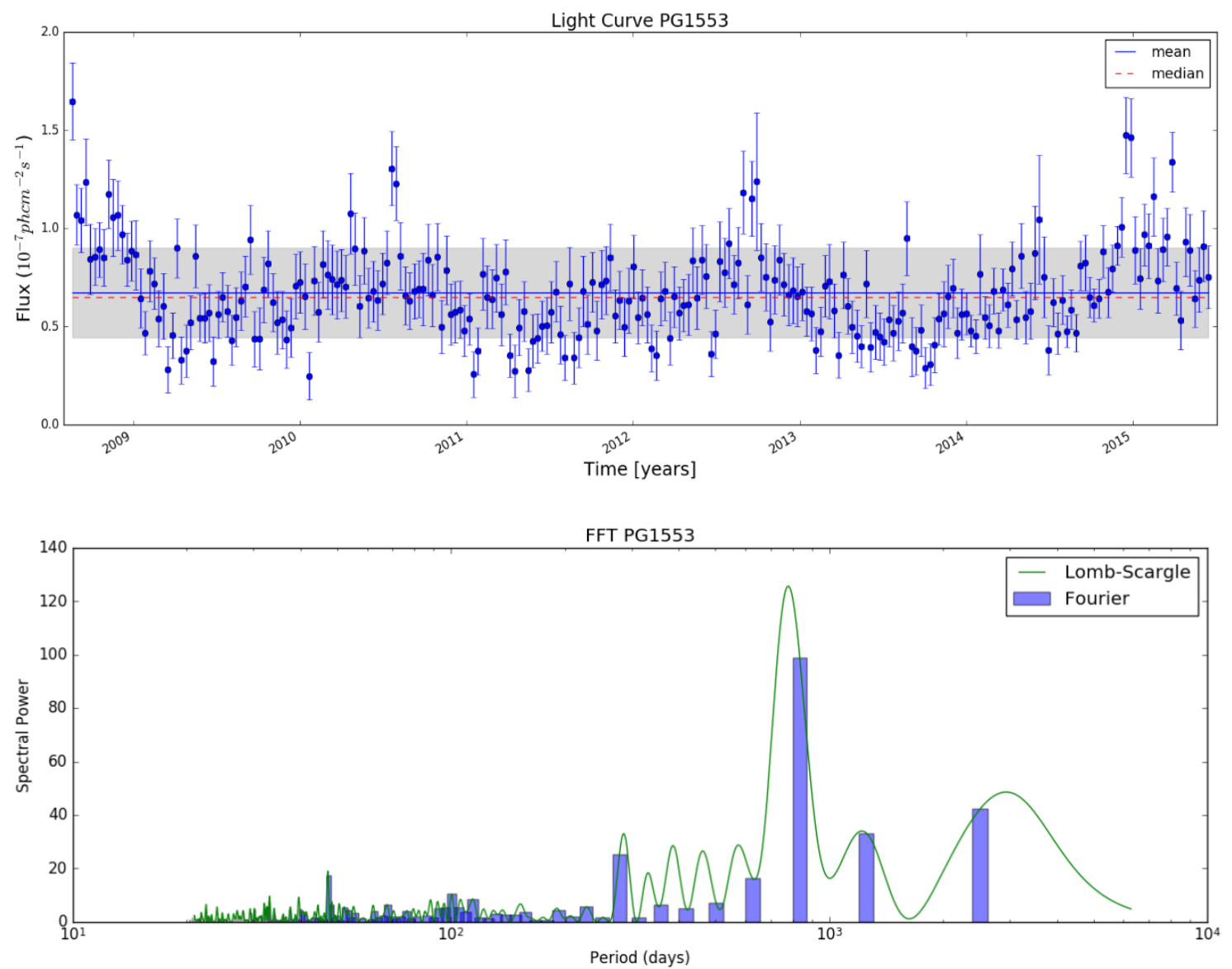

Figura 2.9: curva de luz do PG 1553+113 (acima, com bins temporais de 10 dias) e sua análise espectral. Os picos diferem ligeiramente nos dois métodos: $P_{F F T} \approx 833$ dias e $P_{L S P} \approx 779$ dias, em coerência com o período de $795 \pm 29$ dias, reportado na referência Ackermann et al. (2015b).
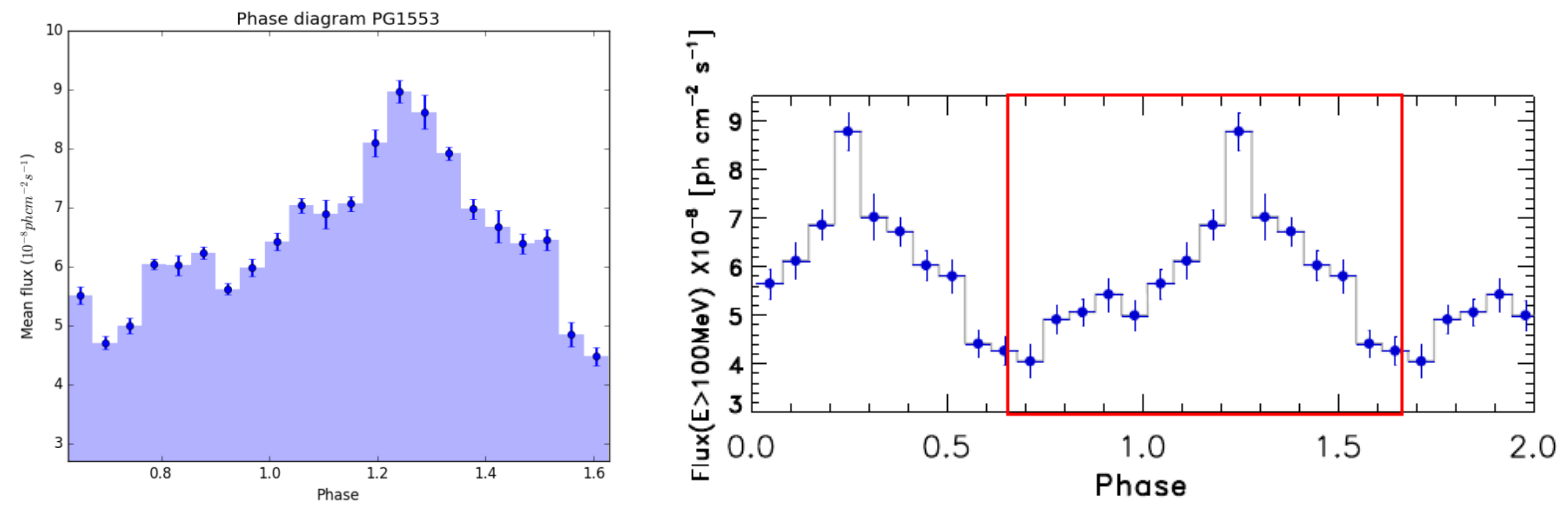

Figura 2.10: diagramas de fase para as curvas de luz em raios- $\gamma$ do PG1553+113. O resultado obtido (esquerda), onde a fase foi definida como $\phi=\left(t-t_{0}\right) / P_{L S P}$, pode ser comparado diretamente com o resultado dentro da caixa vermelha do gráfico da direita, adaptado de Ackermann et al. (2015b). Apesar do número diferente de bins, os dois diagramas deixam clara uma distribuição assimétrica em torno do pico.

tempo e requer que o sinal seja estacionário. A CWT, por outro lado, é capaz de medir as variações da frequência ao longo do tempo e não requer que o sinal seja estacionário. 
Podemos interpretar a CWT como uma versão generalizada da transformada de Fourier. Assim como a transformada de Fourier decompõe sinais estacionários em combinações lineares de senos e cossenos, a CWT decompõe sinais não-estacionários em combinações lineares de wavelets (Liu, 1999).

Suponhamos uma função $X(t)$ de quadrado integrável. Sua transformada de Fourier, $\hat{X}(\omega)$, é dada por

$$
\hat{X}(\omega)=\int_{-\infty}^{\infty} X(t) e^{-i \omega t} d t
$$

que transforma uma função no domínio temporal para o domínio de frequências. Para examinar as características da função tanto no domínio de frequências quanto no domínio do tempo, podemos estender a equação 2.7 para o domínio tempo-frequência incluindo uma função $g(t)$ tal que

$$
\hat{X}(\omega, \tau)=\int_{-\infty}^{\infty} X(t) g(t-\tau) e^{-i \omega t} d t
$$

A equação 2.8, conhecida como transformada de Fourier de janela, pode ser discretizada definindo-se o tempo $\tau$ e a frequência $\omega$ como $a \tau_{0}$ e $b \omega_{0}$, onde $a$ e $b$ são reais e $\tau_{0}, \omega_{0}>0$. Então

$$
\hat{X}(a, b)=\int_{-\infty}^{\infty} X(t) g_{a b}(t) d t
$$

com

$$
g_{a b}(t)=g\left(t-a \tau_{0}\right) e^{-i b \omega_{0} t}
$$

Substituir $g_{a b}(t)$ por uma nova família de funções

$$
\Psi_{a b}(t)=|a|^{-1 / 2} \Psi\left(\frac{t-b}{a}\right)
$$

nos leva diretamente à wavelet transform:

$$
\tilde{X}(a, b)=\int_{-\infty}^{\infty} X(t)|a|^{-1 / 2} \Psi^{*}\left(\frac{t-b}{a}\right) d t
$$

onde $a>0,-\infty<b<\infty$, e o asterisco indica o complexo conjugado. $\Psi(t)$ é conhecida como mother wavelet. O fator de normalização $|a|^{-1 / 2}$ garante que $\Psi_{a b}$ tenha norma constante,

$$
\left\|\Psi_{a b}(t)\right\|=\int_{-\infty}^{\infty}\left|\Psi_{a b}(t)\right|^{2} d t=1
$$

no espaço das funções de quadrado integrável. Para que a função $\Psi$ possa ser uma mother wavelet, ela precisa satisfazer a seguinte condição de admissibilidade:

$$
C_{\Psi}=\int_{-\infty}^{\infty} \frac{|\hat{\Psi}(\omega)|^{2}}{|\omega|} d \omega<\infty,
$$


de tal forma que o sinal/função, $X(t)$, possa ser reconstruído a partir do wavelet, $\Psi_{a b}(t)$, e sua correspondente wavelet transform, $\tilde{X}(a, b)$, como

$$
X(t)=\frac{1}{C_{\Psi}} \int_{-\infty}^{\infty} \int_{-\infty}^{\infty} \tilde{X}(a, b) \Psi_{a b}(t) \frac{d a d b}{b^{2}}
$$

Além disso, uma wavelet $\Psi(t)$ deve ser uma função que oscila num curto intervalo de tempo e tenha média zero. As equações 2.9 a 2.12 essencialmente resumem a análise por CWT. Mais detalhes podem ser conferidos nas referências Torrence and Compo (1998) e Liu (1999).

A CWT transforma uma função ou sinal de uma variável independente $t$ em uma função de duas variáveis independentes $a$ e $b$. Diferente da transformada de Fourier, que é restrita a uma base de senos e cossenos, podemos escolher muitas formas distintas de mother wavelet para a CWT. Uma das mais conhecidas (e adotada neste trabalho) é a wavelet de Morlet (figura 2.11), definida como uma onda plana modulada por uma gaussiana:

$$
\Psi(t)=\pi^{-1 / 4} e^{i \omega_{0} t} e^{-t^{2} / 2}
$$

onde $\omega_{0}$ é a frequência adimensional, escolhida de tal forma que a condição de admissibilidade (eq. 2.11) seja satisfeita (Liu, 1999).

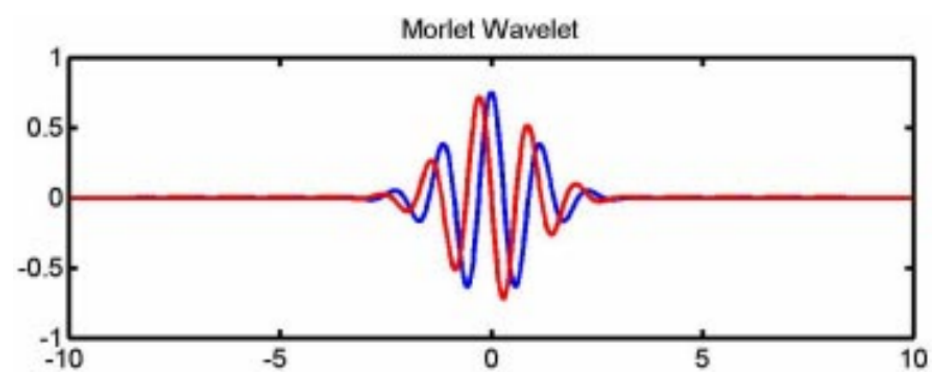

Figura 2.11: componentes real (azul) e imaginária (vermelho) da Morlet wavelet. Fonte: Liu (1999).

Nossa análise por CWT da curva de luz (figura 2.9) do PG1553+113 é mostrada na figura 2.12. Aqui, consideramos o sinal cíclico, ou seja, supomos que o sinal observado se repete indefinidamente no tempo, de modo a evitar os efeitos de borda (tentamos reproduzir o resultado da referência Ackermann et al. (2015b) da forma mais fiel possível). Assumir que o sinal é cíclico, no entanto, pode gerar frequências espúrias nos nossos dados. Voltaremos à análise do PG1553+113 no capítulo 3, onde os efeitos de borda serão levados em conta.

O red noise mostrado na figura 2.12 possui densidade espectral que cresce na direção dos longos comprimentos de onda (Rudnick and Davis, 2003), o que significa que este ruído possui maior energia em baixas frequências. Devemos levá-lo em consideração sempre que o período em nossos dados for comparável ao tamanho de nossa série temporal (Rudnick 

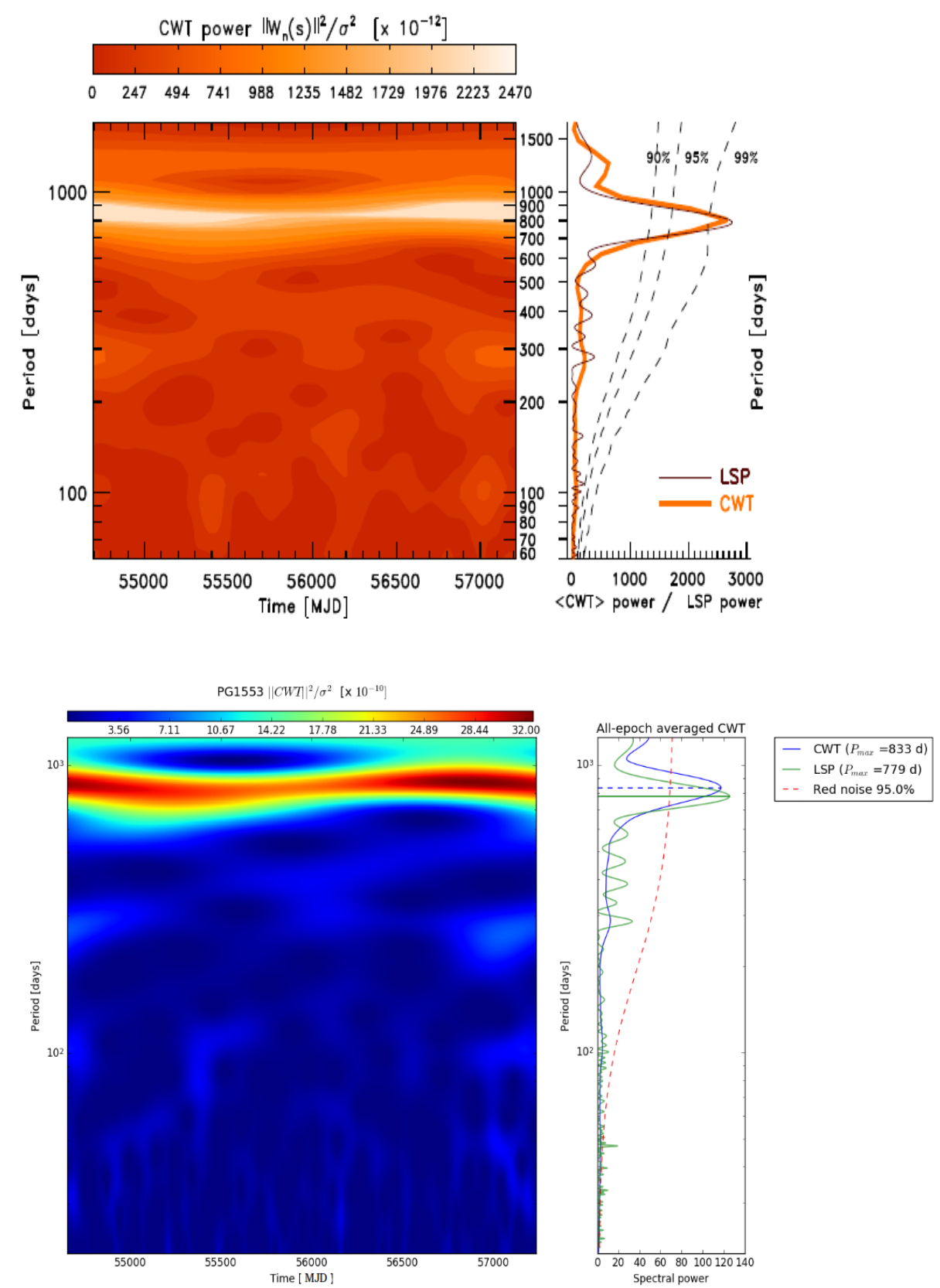

Figura 2.12: espectro de potência (ou periodograma) da CWT da curva de luz (figura 2.9) do PG1553+113 usando uma Morlet mother wavelet. O painel à direita de cada imagem representa a média temporal do espectro de potência da CWT, assim como o LSP das respectivas curvas de luz em raios $\gamma$. Acima: resultado de Ackermann et al. $(2015 \mathrm{~b})$. Abaixo: resultado obtido.

and Davis, 2003) e podemos estimá-lo por simulações de Monte Carlo (Ackermann et al., 2015b; Rudnick and Davis, 2003) ou por equações empíricas (Torrence and Compo, 1998). Neste projeto adotamos a relação empírica

$$
P_{k}=\frac{1-\alpha^{2}}{1+\alpha^{2}-2 \alpha \cos (2 \pi k / N)}
$$


onde $P_{k}$ é o espectro de Fourier do red noise, $k=0,1, \ldots, N / 2$ é o índice correspondente a cada uma das possíveis frequências (reais e positivas) do nosso sinal, $N$ é o número total de frequências computadas na FFT (incluindo as frequências negativas) e $\alpha$ é o coeficiente de autocorrelação $\operatorname{lag}-1{ }^{13}$, convencionalmente escolhido como $\alpha=0,72$ (Torrence and Compo, 1998) para este tipo de análise.

${ }^{13}$ Ver a referência Torrence and Compo $(1998)$ para mais detalhes. 


\section{CAPÍTULO 3}

\section{Resultados}

A maioria da população de LLAGN $(\approx 2 / 3)$ é composta por LINERs, sistemas que se encontram muito abaixo do limite de Eddington, com uma razão $L / L_{E d d}$ média de $10^{-5}(\mathrm{Ho}, 2009)$. As propriedades observacionais de LLAGNs favorecem um cenário para seu motor central bem diferente dos AGNs mais luminosos: o SMBH em seus centros está acretando matéria em baixa taxa no modo de Advection-Dominated Accretion Flow (ADAF), que parece ser bastante eficiente na produção de jatos e outflows (Nemmen and Tchekhovskoy, 2015). De fato, a maioria dos LLAGNs possuem jatos na escala de parsecs, que estão no limite de resolução dos radiotelescópios atuais (Mezcua and Prieto, 2014).

Ainda não é claro qual fator astrofísico — jato ou escoamento de acreção — é responsável pela emissão em altas energias observada nestas galáxias. Na literatura, raios $\gamma$ observados em AGNs são comumente associados à presença de jatos, como é o caso dos blazares, entretanto, para os LINERs, esperamos que o plasma quente em ambos o ADAF e o jato irradiem raios $\gamma$ através de decaimento de píons (Mahadevan et al., 1997) ou SSC.

Neste capítulo resumiremos nossos resultados, onde discutiremos sobre as propriedades físicas das galáxias de nossa amostra e das restrições obtidas para o tamanho de suas regiões emissoras de raios $\gamma$. Discorreremos ainda sobre o comportamento aparentemente periódico dos AGNs PG 1553+113 e NGC 1275 e sobre evidências de novas fontes de raios $\gamma$.

\subsection{Amostra}

O Palomar sample é um survey espectroscópico da região nuclear $(r \lesssim 200$ pc) de um conjunto de 486 galáxias do Universo local. Seus espectros ópticos de fenda longa foram obtidos do núcleo de quase toda galáxia brilhante $(B<12,5 \mathrm{mag})$ do hemisfério norte 


\begin{tabular}{l|c|c|c|c|l} 
LLAGN & TS & redshift & Dist. $(\mathrm{Mpc})$ & SMBH $\left(M_{\odot}\right)$ & Referência \\
\hline NGC 1275 & 42411,83 & 0,017559 & 69,767 & $3,4 \times 10^{8}$ & Wilman et al. $(2005)$ \\
NGC 6503* & 3806,25 & 0,000083 & 5,664 & $\lesssim 2 \times 10^{6}$ & Kormendy et al. $(2010)$ \\
NGC 4486 & 637,86 & 0,004283 & 16,611 & $6,6 \times 10^{9}$ & Gebhardt et al. (2011) \\
NGC 4501* & 72,76 & 0,007609 & 18,841 & $7,8 \times 10^{7}$ & Merloni et al. (2003) \\
NGC 4762 & 36,34 & 0,003289 & 16,263 & - & - \\
NGC 3627 & 19,54 & 0,002425 & 9,756 & $1,8 \times 10^{7}$ & Merloni et al. (2003) \\
NGC 4651 & 18,89 & 0,002628 & 23,700 & - & - \\
NGC 3623 & 18,50 & 0,002692 & 12,771 & $1,2 \times 10^{7}$ & Dong and De Robertis $(2006)$ \\
NGC 3301 & 14,36 & 0,004466 & 25,150 & $3,1 \times 10^{7}$ & Dong and De Robertis $(2006)$ \\
NGC 2541 & 13,91 & 0,001828 & 12,311 & - & - \\
NGC 1055 & 13,33 & 0,003314 & 16,771 & $1,0 \times 10^{7}$ & Dong and De Robertis $(2006)$ \\
NGC 4261 & 12,45 & 0,007378 & 29,912 & $4,9 \times 10^{8}$ & Ferrarese et al. $(1996)$ \\
NGC 410 & 11,75 & 0,017659 & 66,000 & - & - \\
NGC 315 & 8,87 & 0,016485 & 56,014 & - & - \\
NGC 5371 & 8,57 & 0,008533 & 29,483 & - & -
\end{tabular}

Tabela 3.1: alvos analisados e seus respectivos valores de TS (calculados com base nos sete primeiros anos de dados do telescópio), redshift, distância e massa do SMBH. As fontes marcadas com *, apesar de estarem dentro da PSF do sinal, estão associadas a blazares pelo 3FGL, o terceiro catálogo de fontes do Fermi (Acero et al., 2015b) e foram excluídas da análise. A associação dos TS com seus respectivos LLAGNs só é garantida para as NGC 1275 e NGC 4486, as outras fontes carecem de uma análise mais detalhada em outros comprimentos de onda. Os erros associados aos valores de massa tabelados acima podem ser conferidos nas suas respectivas referências ou adiante no texto. Os redshifts e distâncias foram obtidos do NASA/IPAC Extragalactic Database (NED), sob a coordenação do Jet Propulsion Laboratory, California Institute of Tecnology, em contrato com a NASA.

$\left(\delta>0^{\circ}\right)$ (Ho et al., 1997a) e sua classificação espectral nos fornece inúmeras informações sobre a demografia da atividade em núcleos de galáxias nas vizinhanças da Via Láctea.

Os AGNs do Palomar sample possuem uma luminosidade $H \alpha$ (corrigida por extinção) média de apenas $2 \times 10^{39} \mathrm{erg} \mathrm{s}^{-1}$ (Ho et al., 1997a), e aproximadamente $85 \%$ das fontes emitem menos de $L(H \alpha)=10^{40} \operatorname{erg~s}^{-1}$ (Ho et al., 1997b). Em contraste, os núcleos de Seyfert típicos no catálogo de Markarian emitem cerca de $10^{41} \mathrm{erg} \mathrm{s}^{-1}$ em $H \alpha(\mathrm{Ho}$ et al., 1997a). No Palomar sample, LLAGNs são definidos como aqueles em que $L(H \alpha) \leq$ $10^{40} \operatorname{erg~s}^{-1}$ (Ho et al., 1997a).

Um mapa de TS foi construído para cada um dos cerca de 200 AGNs presentes no Palomar sample (Nemmen et al., em preparação) e escolhemos as 15 fontes com maior significância estatística para fazer nossa análise (tabela 3.1). Dentre estas fontes, porém, apenas 5 foram brilhantes o suficiente para podermos construir curvas de luz. São elas: NGC 1275 (Perseus A), NGC 4486 (M87), NGC 3301, NGC 4762 e NGC 3627/NGC 3623 (estas duas galáxias encontram-se muito próximas no plano do céu - a menos de $0,5^{\circ}$ de distância uma da outra - e um único sinal de raios $\gamma$ foi detectado no campo de visão que engloba as duas galáxias. Mais detalhes na seção 3.2). 


\subsection{Curvas de luz e mapas de TS}

Nas curvas de luz mostradas a seguir, as barras de erro representam o intervalo de $1 \sigma$ de confiança, a região hachurada representa o desvio padrão calculado usando toda a série temporal, que nos ajuda a diferenciar o que é, de fato, flare, e o que é flutuação; enquanto que a mediana das medidas foi adotada como estado quiescente dos alvos.

As escalas de tempo de variabilidade, $t_{v a r}$, com o fluxo dobrando ou caindo pela metade, foram usadas para a estimativa do tamanho da região emissora de acordo com a equação

$$
d \leq \frac{2 c t_{v a r}}{(1+z)}
$$

que nos diz qual o tamanho máximo que uma região emissora pode ter sem quebrar o princípio da causalidade - a região deve ser menor que a distância que a luz percorreu durante o tempo de variabilidade.

Frequentemente, o fluxo em alguns bins de tempo não foi significativamente diferente de zero. Então, sempre que o sinal possuía $T S \leq 3$ (90\% de confiança) ou quando havia problemas na convergência da MLE, calculamos limites superiores (95\%). Para isso, usamos a função de distribuição de probabilidade do parâmetro, $\theta$, de interesse, obtida da normalização da likelihood, $L$, para o intervalo de valores permitidos do parâmetro $\theta$. A probabilidade $P\left(\theta<\theta_{0}\right)$ computada desta densidade é o nível de confiança, $C$, do limite superior $\theta_{0}$ :

$$
C\left(\theta_{0}\right)=\frac{\int_{-\infty}^{\theta_{0}} L(\theta) d \theta}{\int_{-\infty}^{\infty} L(\theta) d \theta} .
$$

Escolhendo o nível de confiança como $C=0,95$, a equação 3.2 deve ser resolvida para $\theta_{0}$ (Bohm and Zech, 2010).

A maioria das fontes da tabela 3.1 não estão catalogadas no 3FGL (third Fermi Gamma-ray LAT catalog), que cobre os primeiros quatro anos de dados do telescópio, e, quando necessário, foram adicionadas manualmente ao modelo xml de entrada com o espectro seguindo uma lei de potência, do mesmo modo que é mostrado na figura 2.2 .

\subsubsection{Perseus A}

A galáxia ativa Perseus A (ou NGC 1275), Seyfert do tipo 211 , distante aproximadamente $70 \mathrm{Mpc} \mathrm{L}^{2}$ e localizada no centro do aglomerado de Perseus, é uma conhecida fonte de rádio e forte emissora de raios X (Falceta-Gonçalves et al., 2010; Fabian et al., 2011).

\footnotetext{
${ }^{1}$ SIMBAD Astronomical Database: Perseus+A. Acesso em 18/07/2016.

${ }^{2} \mathrm{NED}: \quad$ http://ned.ipac.caltech.edu/cgi-bin/nDistance?name=NGC+1275. Acesso em $18 / 07 / 2016$. 


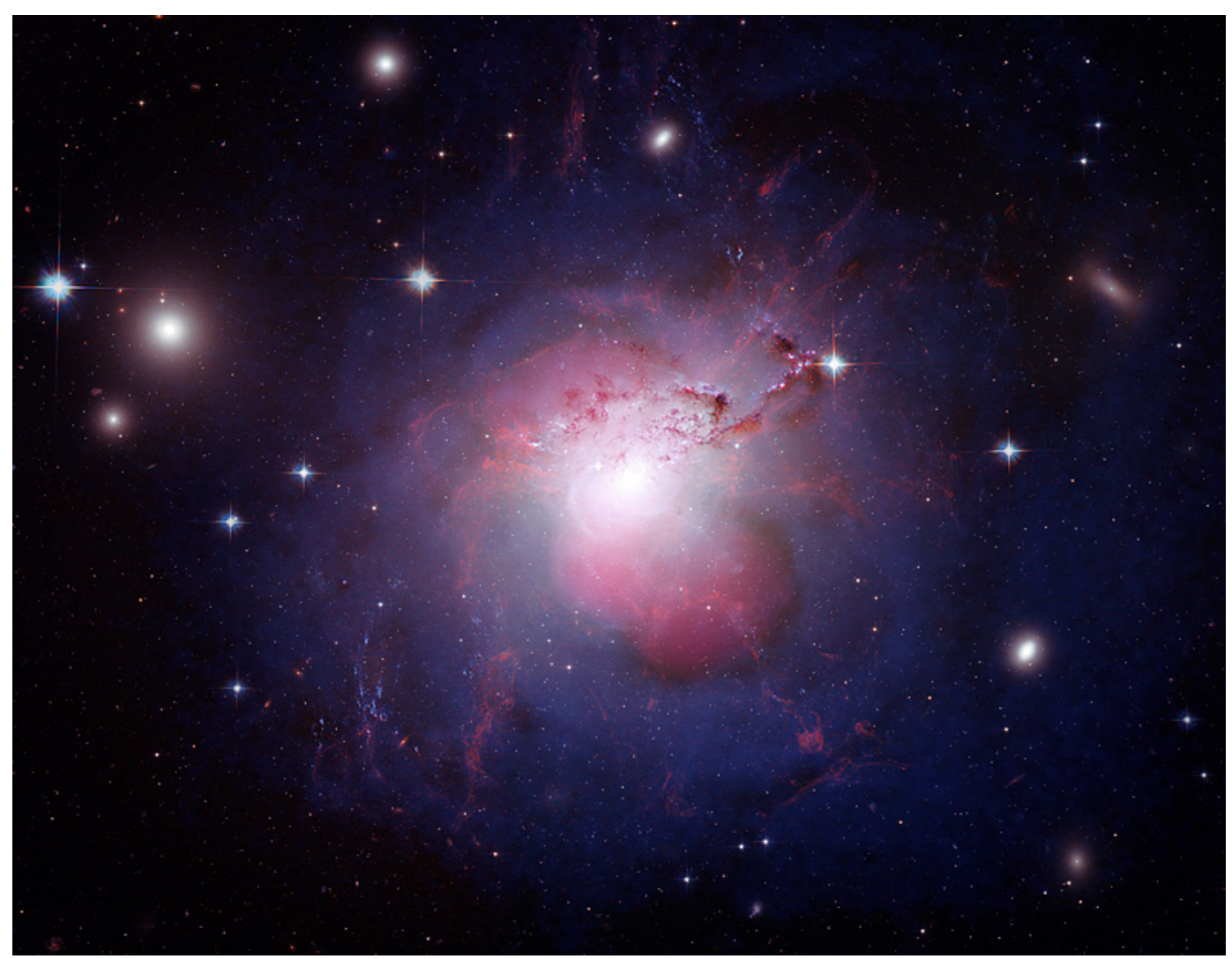

Figura 3.1: imagem composta da galáxia Perseus A: os dados em raios $\mathrm{X}$, obtidos com o telescópio espacial Chandra, aparecem como tênues cascas azuis. Os lóbulos em rosa, saindo do centro da galáxia, foram observados em rádio pelo NRAO Very Large Array. As faixas de poeira, regiões de formação de estrelas, filamentos de hidrogênio, estrelas de primeiro plano e galáxias de fundo foram obtidas no óptico pelo HST. Esta imagem envolve um campo de $3,87^{\prime} \times 3,87^{\prime}$. Fonte: raios X: NASA/CXC/IoA/Fabian et al. (2006).; Rádio: NRAO/VLA/G. Taylor; Óptico: NASA/ESA/Hubble Heritage (STScI/AURA) \& Univ. of Cambridge/IoA/A. Fabian.

Sua emissão em raios X forma um par de bolhas (em azul na figura 3.1) com dimensões de quiloparsecs posicionadas em ângulos relativamente diferentes com respeito aos jatos observados em rádio, mostrados em rosa na figura 3.1 (Falceta-Gonçalves et al., 2010). Este desalinhamento é causado, possivelmente, pela precessão dos jatos do AGN num período de $3,3 \times 10^{7}$ anos com um ângulo de semi-abertura em torno de $50^{\circ}$ (Dunn et al. 2006).

Uma outra característica marcante deste AGN, quando observado no óptico, são suas estruturas filamentares. Estes filamentos avermelhados são compostos de gás frio (em comparação com o meio intergaláctico no qual estão imersos), mantidos em equilíbrio provavelmente pela influência de campos magnéticos ${ }^{3}$, e são rodeados por gás quente, emissor de raios X, com algumas dezenas de milhões de Kelvin, no centro do aglomerado de Perseus (Fabian et al., 2008).

${ }^{3}$ Hubble Sees Magnetic Monster in Erupting Galaxy: http://hubblesite.org/newscenter/ archive/releases/2008/28/image/a/. Acesso em 18/07/2016. 
Como a fonte mais brilhante da nossa amostra $(T S \sim 42000)$, a curva de luz em raios $\gamma$ da NGC 1275 mostra variações bruscas na escala de tempo de dez dias (figura 3.2). O tamanho da região emissora foi estimado em $d \leq 0,0165$ pc de acordo com a equação 3.1. onde $z \approx 0.017559$ (tabela 3.1 ) e $t_{v a r}=10$ dias. A massa estimada para o SMBH no centro da Perseus A é $M_{\bullet}=3,4 \times 10^{8} M_{\odot}$, com um erro sistemático de $\pm 0,18$ dex (Wilman et al. 2005 ), que nos dá um raio de Schwarzschild (seção 1.3.5) $r_{s} \approx 3,27 \times 10^{-5}$ pc. Escrevendo o tamanho da região emissora de raios $\gamma$ em termos de $r_{s}$, obtemos $d \lesssim 504 r_{s}$.

Como o índice $\gamma$ do nosso modelo espectral, definido na equação 2.1, variou pouco em relação à média (figura 3.3), este foi mantido constante com valor $\gamma=2,071$ durante a criação das curvas de luz.

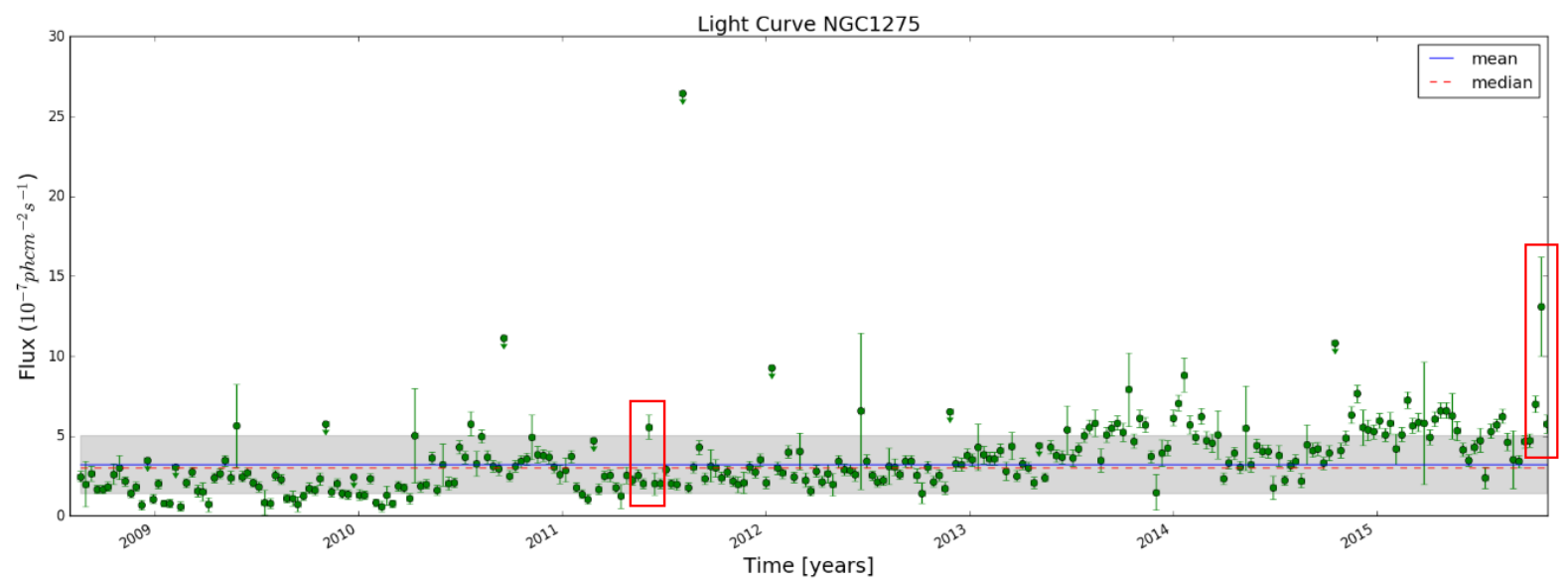

Figura 3.2: curva de luz com bins temporais de dez dias da NGC 1275. Algumas poucas variações bruscas (caixas vermelhas) no fluxo indicam que o tamanho da região emissora de raios $\gamma$ é menor que $0,0165 p c$.

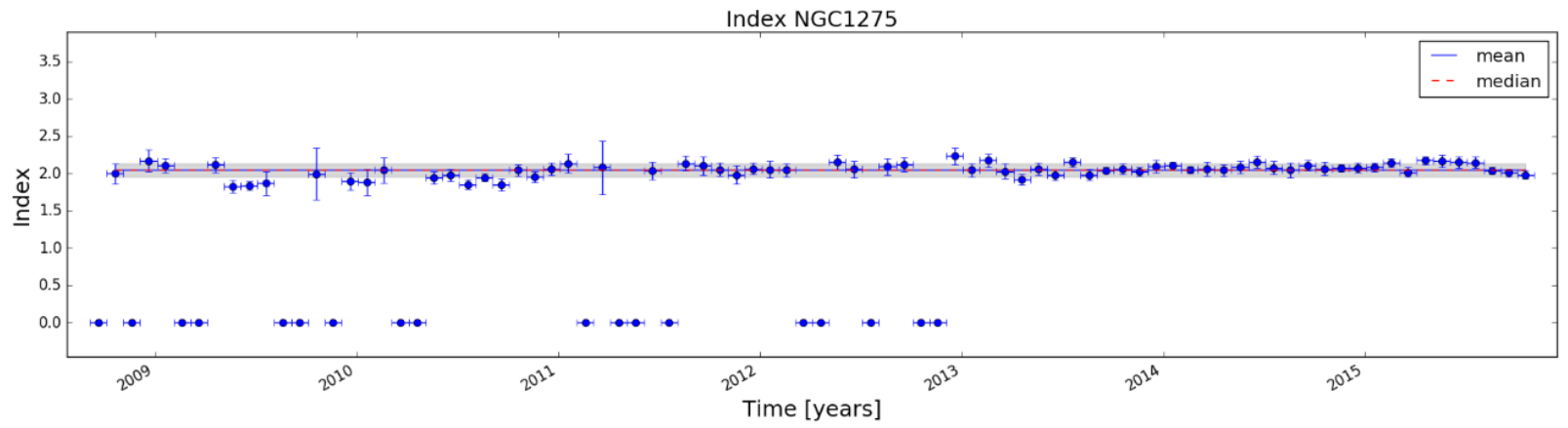

Figura 3.3: variação mensal do índice espectral ao longo dos sete anos de observações. Dada a baixa variância destas medidas, resolvemos fixar o índice no valor de sua mediana, o que facilita a convergência da MLE em cada bin. Nosso algoritmo tem como padrão atribuir o valor zero aos bins que não convergiram. 


\subsubsection{Messier 87}

Localizada a aproximadamente $16,6 \mathrm{Mpc}$ de distância da Terra 4 a rádio galáxia Messier 87 (ou NGC 4486) é uma early-type gigante no centro do aglomerado de Virgem (Schneider, 2014). Esta galáxia é muito conhecida por seu jato óptico (figura 3.4 emanando do seu centro e movendo-se com velocidade aparente superluminal. Seu diâmetro é estimado em $40 \mathrm{kpc}$ e sua massa em $M>3 \times 10^{12} M_{\odot}$ (Schneider, 2014).

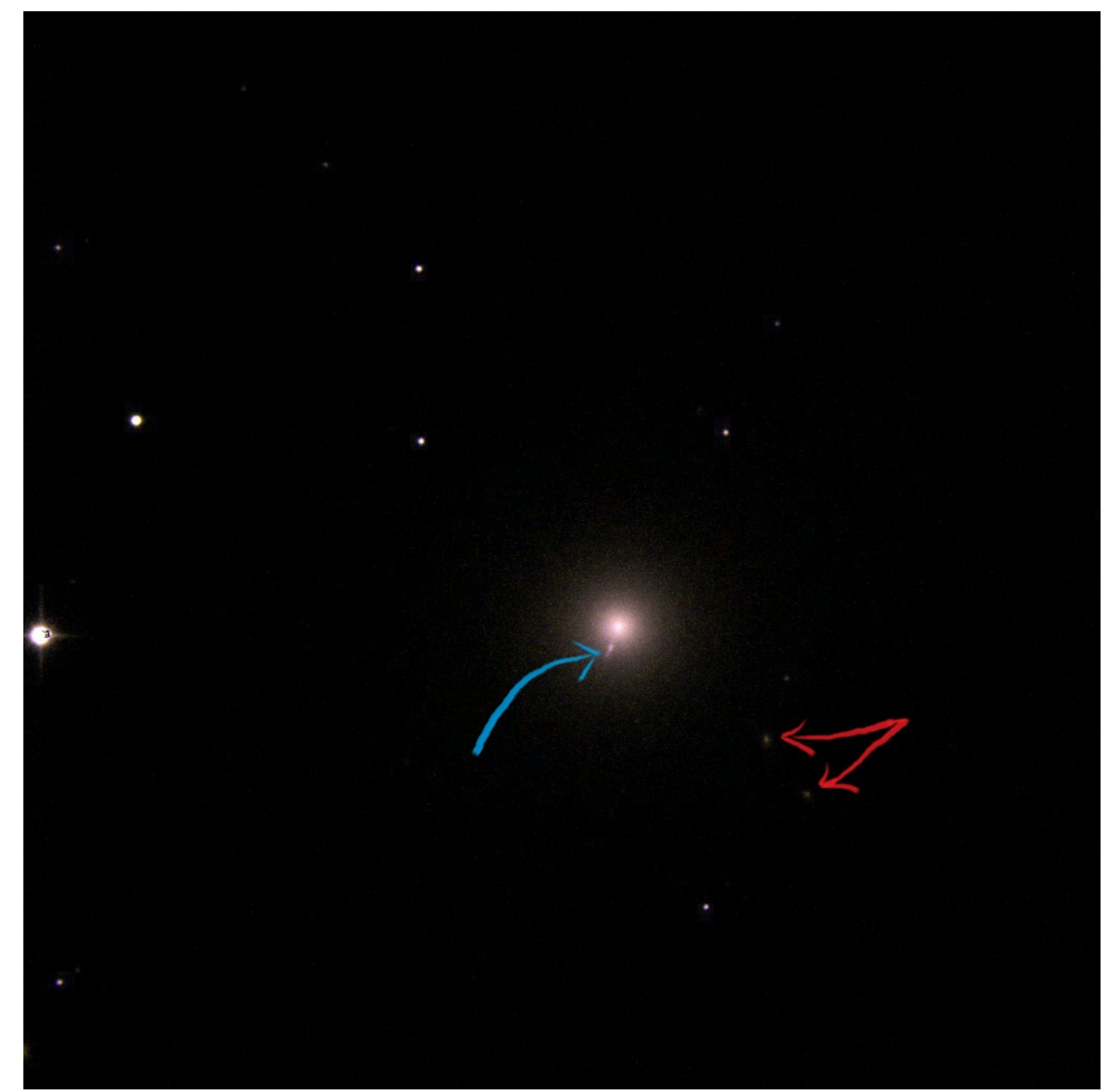

Figura 3.4: imagem no óptico da galáxia M87, onde notamos seu jato relativístico (seta azul) e um par de galáxias conhecido como UGC 7652 (setas vermelhas) que estão muito mais distantes $(z \sim 0,092$, disponível em http://simbad.u-strasbg.fr/simbad/ sim-id?Ident=UGC+7652\&NbIdent=1. Acesso em 19/07/2016.) que a M87 e não fazem parte do aglomerado de Virgem. Ainda notamos, do lado esquerdo, uma estrela de primeiro plano. Imagem com aproximadamente $12^{\prime} \times 12^{\prime}$ obtida com 20 segundos de exposição nos filtros B e V com o telescópio Boller \& Chivens do observatório do Pico dos Dias. Fonte: Raniere Menezes.

Com TS 600, a likelihood da Messier 87 (NGC 4486) dificilmente converge para bins temporais menores que 20 dias e, quando o fazem, as barras de erro são relativamente

\footnotetext{
${ }^{4}$ NED: http://ned.ipac.caltech.edu/cgi-bin/nDistance?name=MESSIER+087. Acesso em $19 / 07 / 2016$.
} 
grandes. Deixamos o índice livre (figura 3.5) e detectamos alguns flares (figura 3.6), restringindo o tamanho da região emissora a $d \leq 0,0335$ pc (usando $z=0.004283-$ tabela 3.1).

Dado que a massa do SMBH no centro da M87 é $M_{\bullet}=(6,6 \pm 0,4) \times 10^{9} M_{\odot}$ (Gebhardt et al. 2011), seu raio de Schwarzschild é $r_{s} \approx 6,34 \times 10^{-4}$ pc, e o tamanho da região emissora em termos de $r_{s}$ é, então, $d \lesssim 53 r_{s}$.

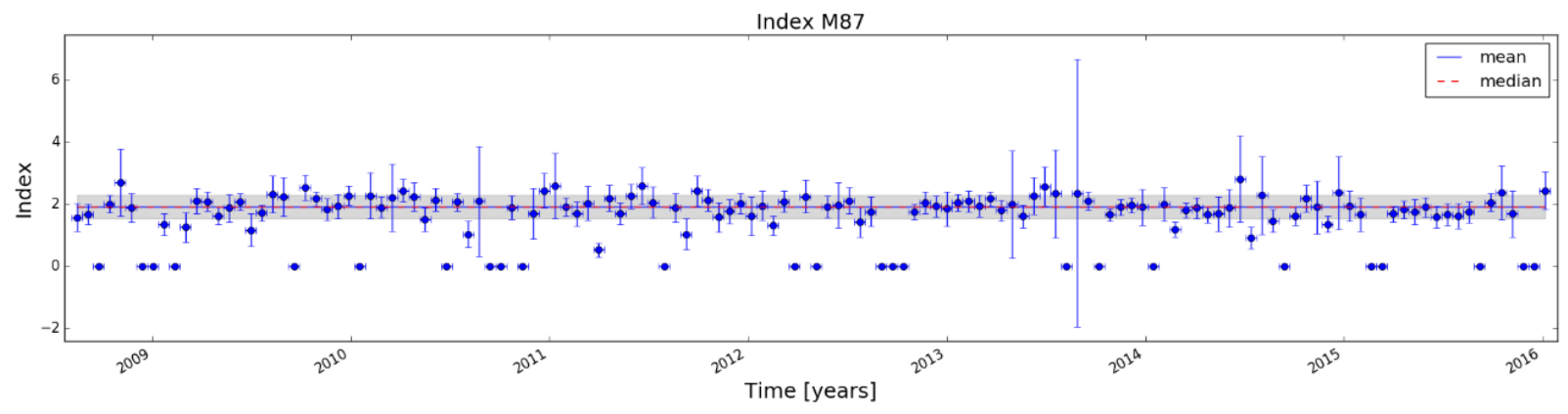

Figura 3.5: variação mensal do índice espectral ao longo dos sete anos de observações. Os bins em que a likelihood não convergiu foram zerados.

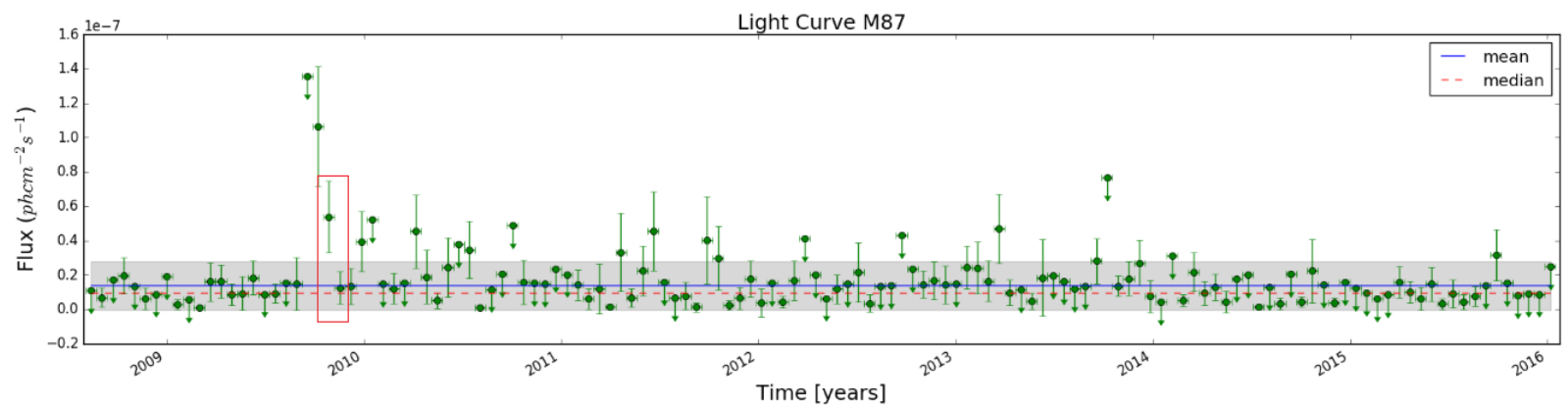

Figura 3.6: curva de luz com bins temporais de vinte dias da M87. O flare indicado pela caixa vermelha implica que o tamanho da região emissora de raios $\gamma$ é menor que 0,0335 pc. Limites superiores foram calculados sempre que o bin não convergia ou quando possuía $T S<3$.

\subsubsection{NGC 3301}

Considerando que o fraco sinal observado em raios $\gamma(T S \sim 20$, figura 3.7) seja, de fato, proveniente da NGC 3301 e usando seu valor de redshift $z=0,004466$ 5 nossa melhor estimativa do tamanho da região emissora foi $d \leq 0,0509 \mathrm{pc}$.

A fonte não está catalogada no 3FGL, mas sua posição espacial coincide com a imagem no óptico do alvo (figura 3.8). Para termos certeza que o sinal vem da NGC 3301, observações em outros comprimentos de onda são indispensáveis.

\begin{tabular}{l}
${ }^{5} \mathrm{NED}: \quad$ https: //ned.ipac. caltech.edu/cgi-bin/objsearch?objname=ngc+3301\&extend=no\& \\
hconst=73\&omegam=0.27\&omegav=0.73\&corr_z=1\&out_csys=Equatorial\&out_equinox=J2000.0\& \\
\hline obj_sort=RA+or+Longitude\&of=pre_text\&zv_breaker=30000.0\&list_limit=5\&img_stamp=YES
\end{tabular}


Devido à baixa significância estatística, forçamos um índice fixo, $\gamma=2,43$, para ajudar na convergência. Escolhemos este valor de acordo com o resultado da binned likelihood aplicada a todo o intervalo de tempo estudado. Nosso resultado é mostrado na figura 3.9 , com um flare de significância acima de $5 \sigma$ (figura 3.10 ) em destaque.

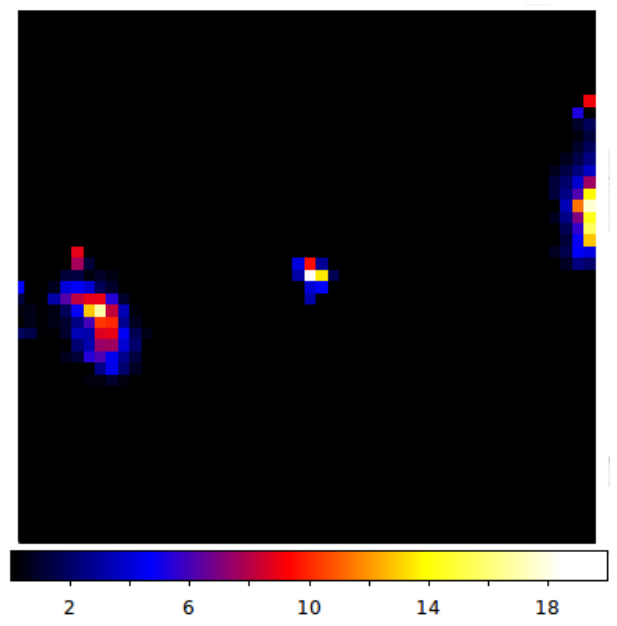

Figura 3.7: mapa de TS com $0,2^{\circ}$ por pixel centrado na NGC 3301. O mapa foi feito com base em sete anos de dados e atinge $T S_{\max }=20,05$ num campo de $10^{\circ} \times 10^{\circ}$. Ainda observamos a presença de outras duas fontes fracas não catalogadas no 3FGL.

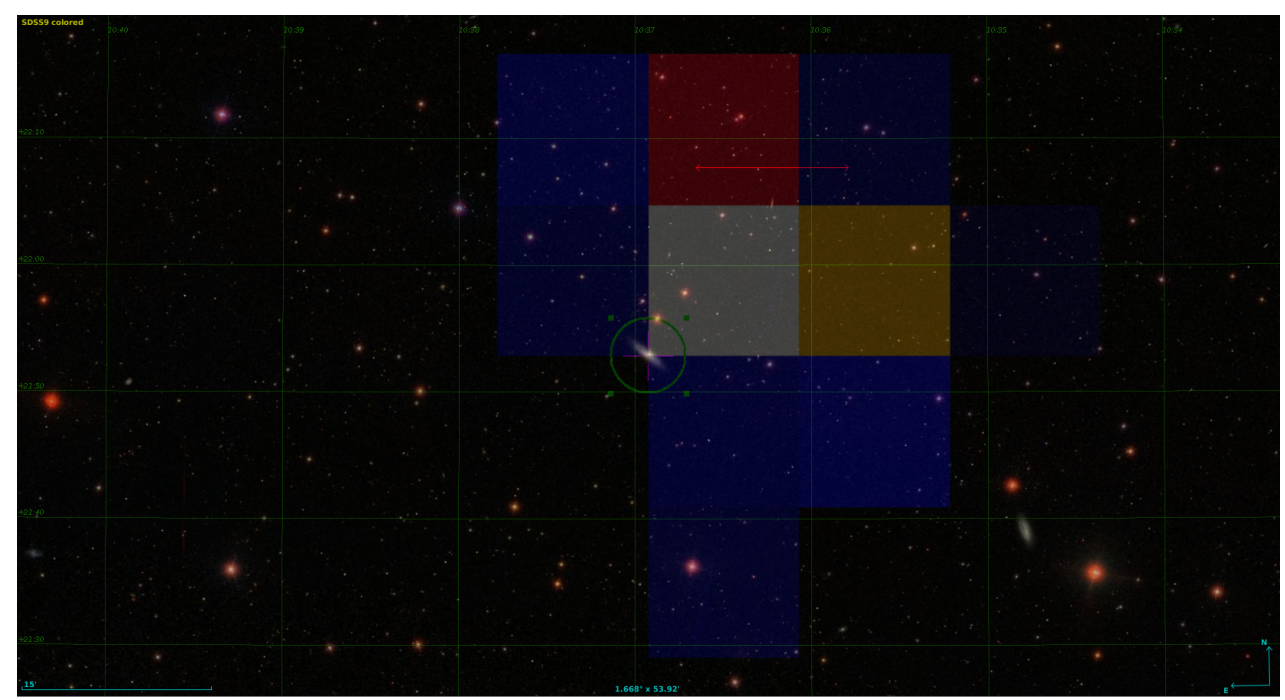

Figura 3.8: sobreposição da imagem do alvo no óptico e seu mapa de TS. Nosso alvo é a galáxia dentro do círculo verde, num campo com $1,668^{\circ} \times 53,92^{\prime}$ centrado em RA = 159,23 , Dec $=21,88$ (J2000.0, Equatorial). A imagem no óptico foi retirada do Aladin Sky Atlas.

Usando o valor da tabela 3.1 para o SMBH da NGC $3301\left(M_{\bullet} \sim 3,1 \times 10^{7} M_{\odot}\right)$, podemos estimar seu raio de Schwarzschild como $r_{s} \approx 2,98 \times 10^{-6} \mathrm{pc}$, e, então, reescrever o tamanho máximo de sua região emissora de raios $\gamma$ como $d \lesssim 17080 r_{s}$. 


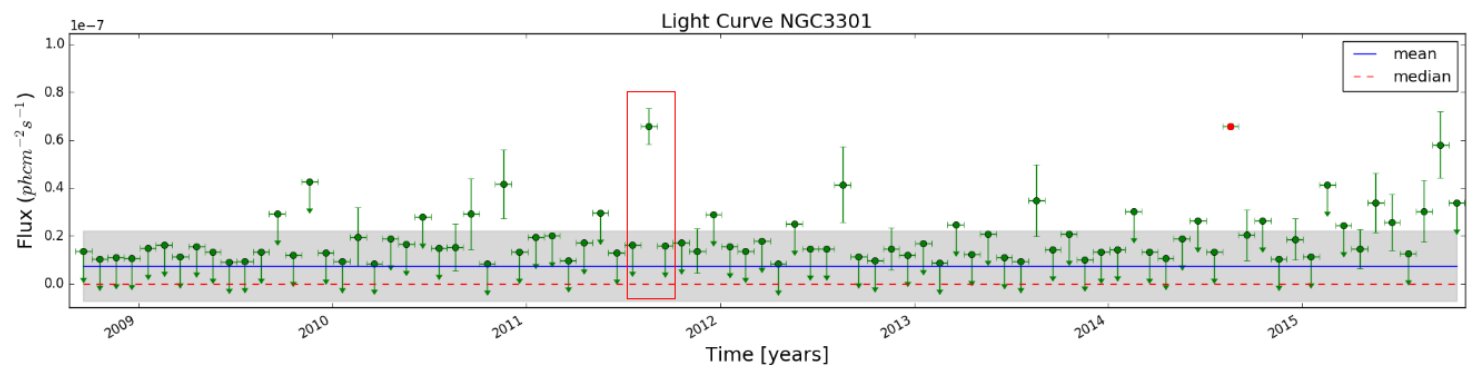

Figura 3.9: curva de luz com bins mensais da NGC 3301. O flare em destaque implica numa região emissora de raios $\gamma \operatorname{com} d \leq 0,0509 p c$. Devido à baixa significância estatística da fonte, é de fundamental importância observarmos a evolução do TS ao longo do tempo. A figura 3.10 deixa claro que o flare observado é significativo. O bin vermelho não é confiável.

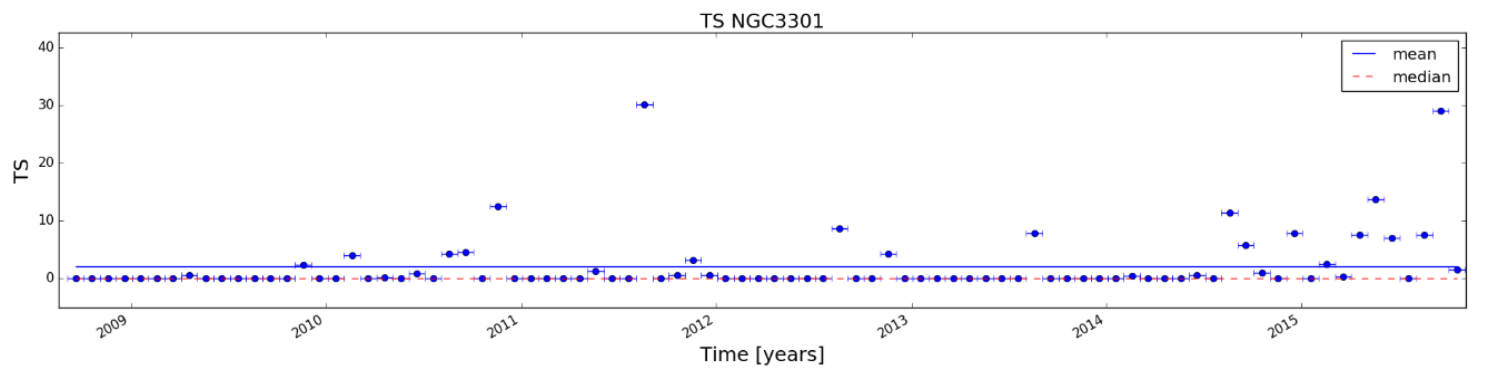

Figura 3.10: TS ao longo do tempo da NGC 3301. Com um valor de TS > 30, o flare em destaque na figura 3.9 possui significância estatística acima de $5 \sigma$.

\subsubsection{NGCs 4762,3627 e 3623}

Diferentemente dos alvos anteriores, o fraco sinal em raios $\gamma$ observado desta vez não estava centrado em nossos alvos, os LINERs 6 NGC 4762, NGC 3627 e NGC 3623, mas em seus arredores (figuras 3.11, 3.12 e 3.13). Apesar dos alvos estarem dentro da PSF, não podemos descartar a hipótese de que o sinal seja proveniente de uma fonte de fundo. Nenhuma das três galáxias está catalogada no 3FGL.

Por estarem muito próximas uma da outra (menos de $0,5^{\circ}$ de distância), as galáxias NGC 3627 e NGC 3623, integrantes do famoso tripleto de Leão, aparecem no mesmo campo na figura 3.13, mas apenas um pico de TS é observado (figuras 3.11b e 3.13) nesta região.

Para os dois sinais observados, fixamos o índice de acordo com o resultado da binned likelihood aplicada a todo o intervalo de tempo estudado, de modo que $\gamma_{N G C 4762}=2,1 \mathrm{e}$ $\gamma_{N G C 3627}=1,88$. As figuras $3.14 \mathrm{e} 3.16$ resumem nossos resultados. Quanto à significância dos flares, plotamos o TS em função do tempo para cada fonte, como mostrado nas figuras 3.15 e 3.17.

\footnotetext{
${ }^{6}$ NGC 4762: http://simbad.u-strasbg.fr/simbad/sim-id?Ident=NGC+4762. NGC 3627: http: //simbad.u-strasbg.fr/simbad/sim-id?Ident=NGC+3627. NGC 3623: http://simbad.u-strasbg. $\mathrm{fr} / \mathrm{simbad} / \mathrm{sim}-\mathrm{id}$ ? Ident=NGC $\$$ \$203623. Acesso em 19/07/2016.
} 


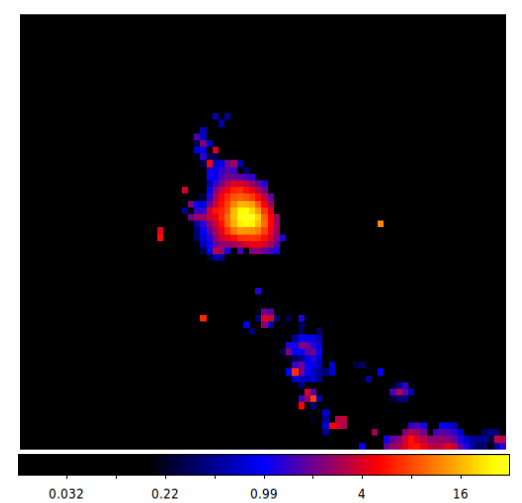

(a) $T S_{\max }=32.2$.

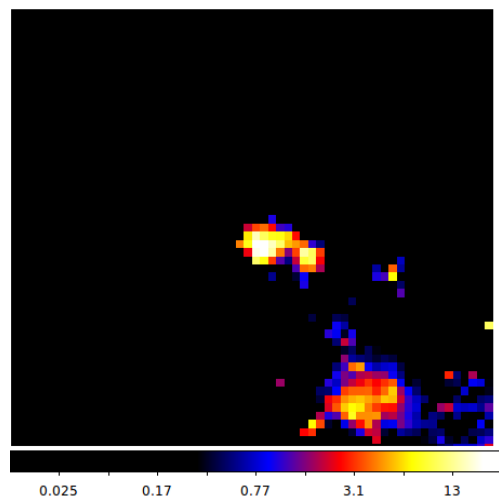

(b) $T S_{\max }=25.1$.

Figura 3.11: mapas de TS com $10^{\circ} \times 10^{\circ}\left(0,2^{\circ}\right.$ por pixel) centrados na NGC 4762 (esquerda) e NGC 3627 (direita). Nos dois casos, o pico de TS está deslocado do centro do campo por cerca de $0,5^{\circ}$. O mapa da direita engloba tanto a NGC 3627 quanto a NGC 3623 (ver figura 3.13 .

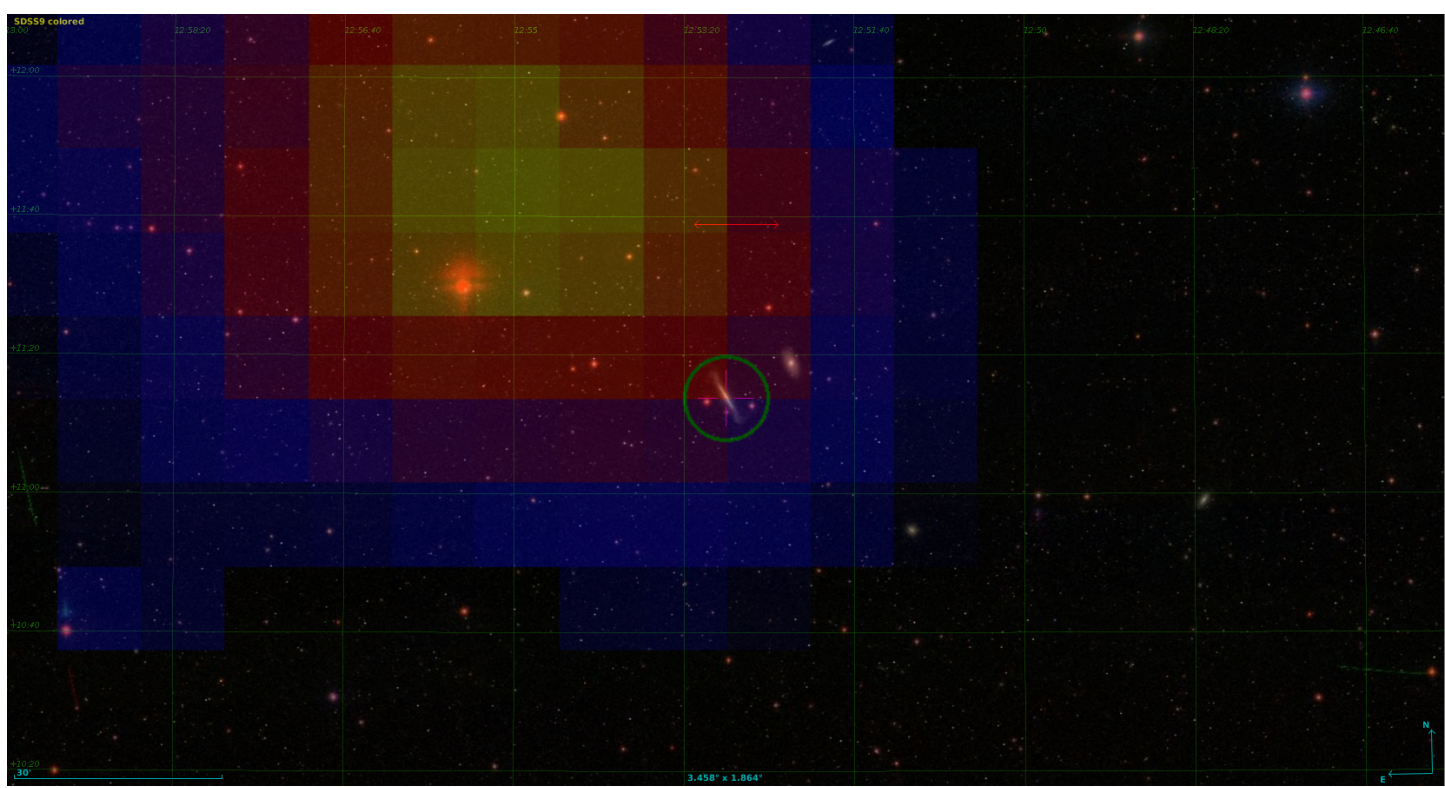

Figura 3.12: sobreposição da imagem no óptico da NGC 4762 (dentro do círculo verde) e seu mapa de TS. O campo possui $3,46^{\circ} \times 1,86^{\circ}$, e está centrado em $R A=193.23$ e $D e c=11.23$ (J2000.0, Equatorial). A NGC 4762 encontra-se dentro do círculo verde. O mapa de TS sobreposto à imagem no ótico é o mesmo da figura 3.11a. A imagem no ótico foi retirada do Aladin Sky Atlas. 


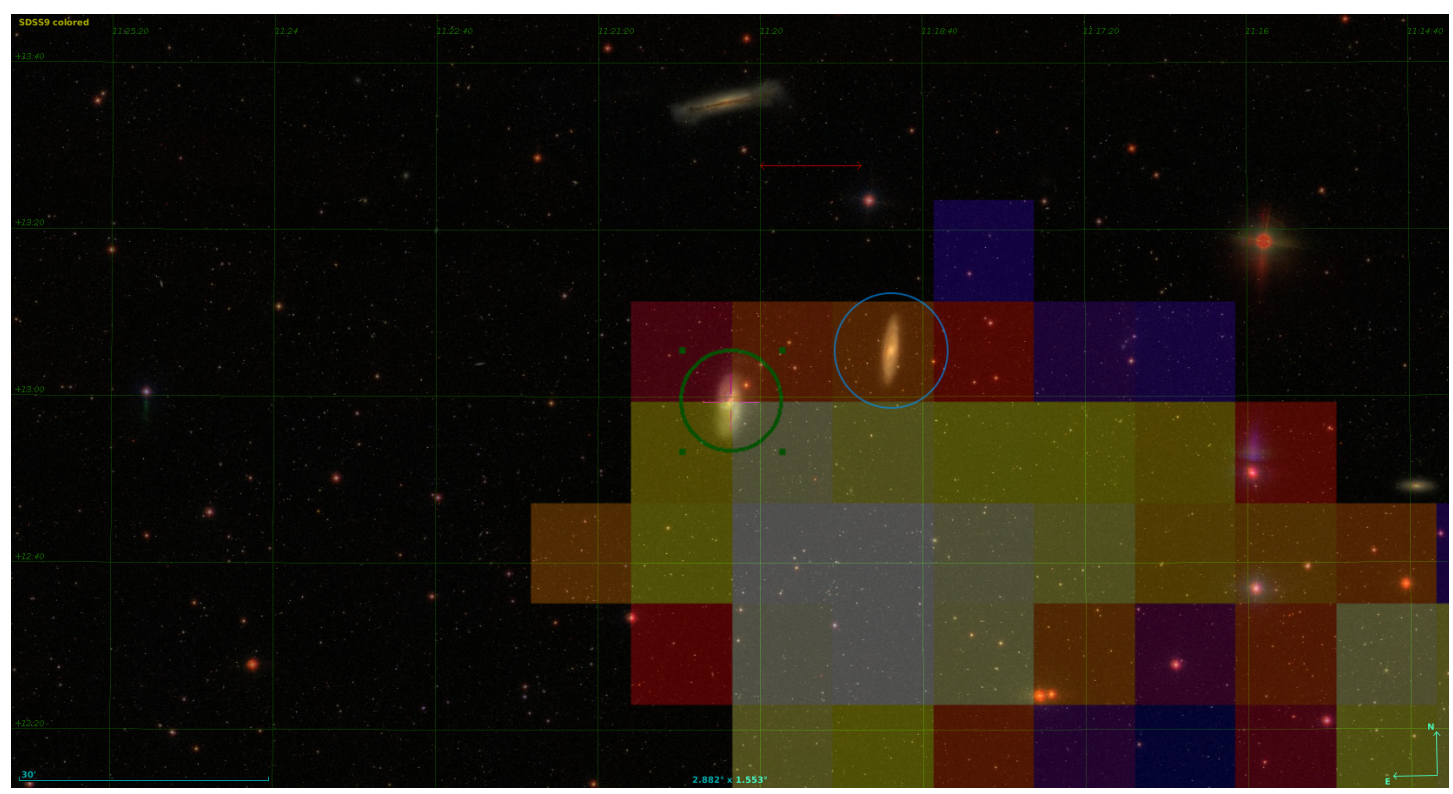

Figura 3.13: sobreposição da imagem das NGCs 3627 (dentro do círculo verde) e 3623 (dentro do círculo azul) no óptico e seu mapa de TS. O campo possui $2,88^{\circ} \times 1,55^{\circ}$, e está centrado em $R A=170.06, D e c=12.99$ (J2000.0, Equatorial). O mapa de TS sobreposto à imagem no óptico é o mesmo da figura 3.11b. A imagem no ótico foi retirada do Aladin Sky Atlas.

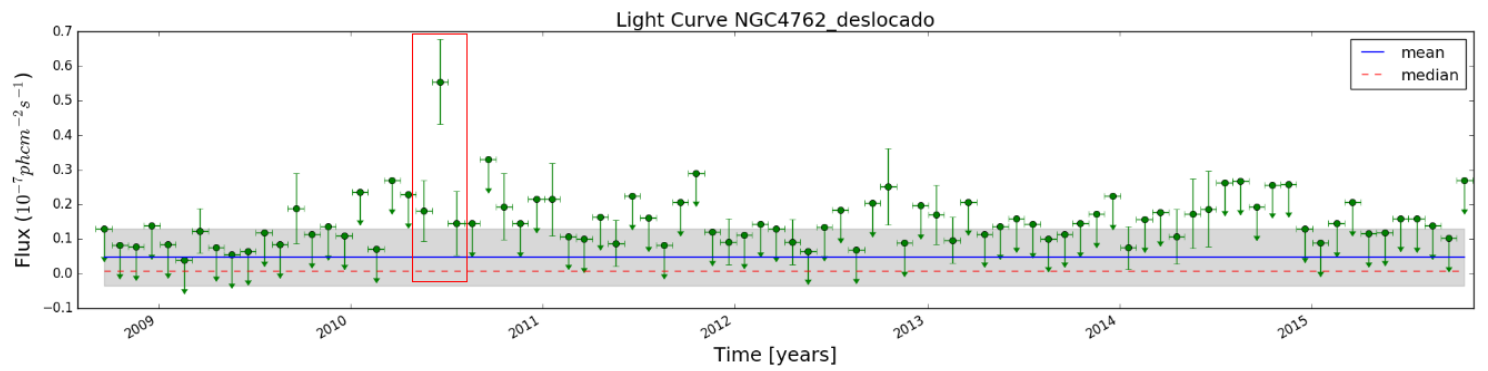

Figura 3.14: curva de luz com bins mensais do sinal observado no campo da NGC 4762. O flare em destaque restringe o light crossing time scale a um mês. A significância do flare pode ser observada na figura 3.15. Para a criação desta curva de luz, fixamos o índice espectral em $\gamma=2,1$.

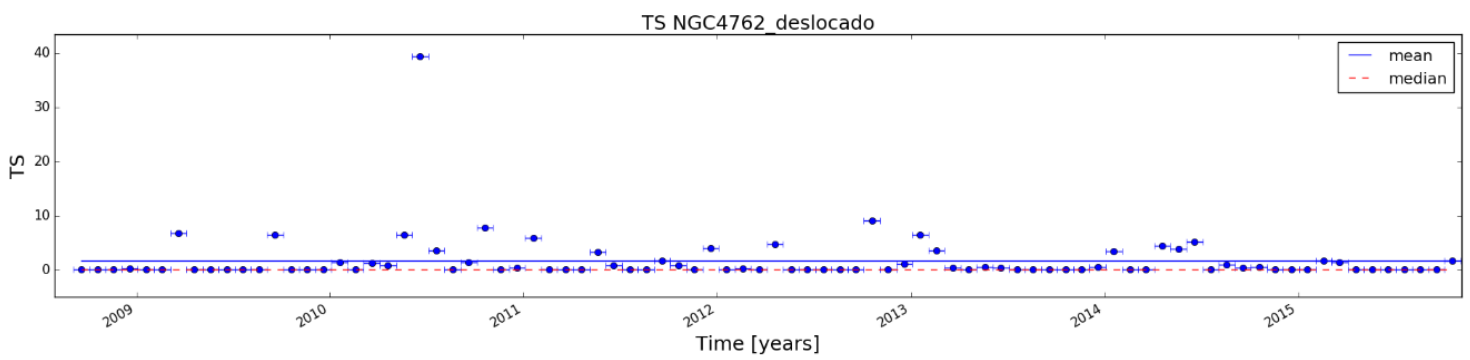

Figura 3.15: TS ao longo do tempo do sinal observado no campo da NGC 4762. Com um valor de $T S>36$, o flare em destaque na figura 3.14 possui significância estatística acima de $6 \sigma$. 


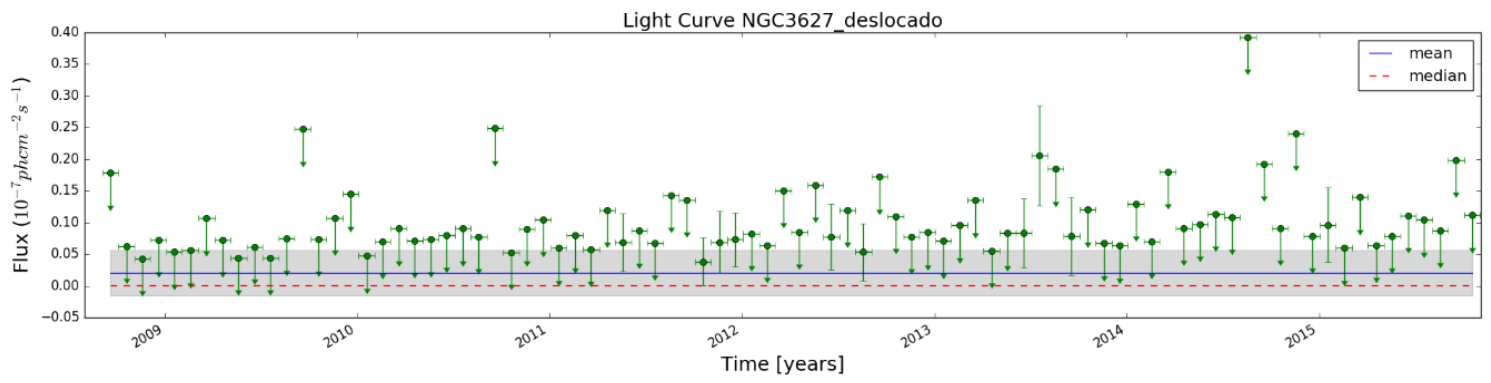

Figura 3.16: curva de luz com bins mensais do sinal observado no campo das galáxias NGC 3627 e NGC 3623. Nenhum flare significativo (ver figura 3.17) foi detectado. Para a criação desta curva de luz, fixamos o índice espectral em $\gamma=1,88$.

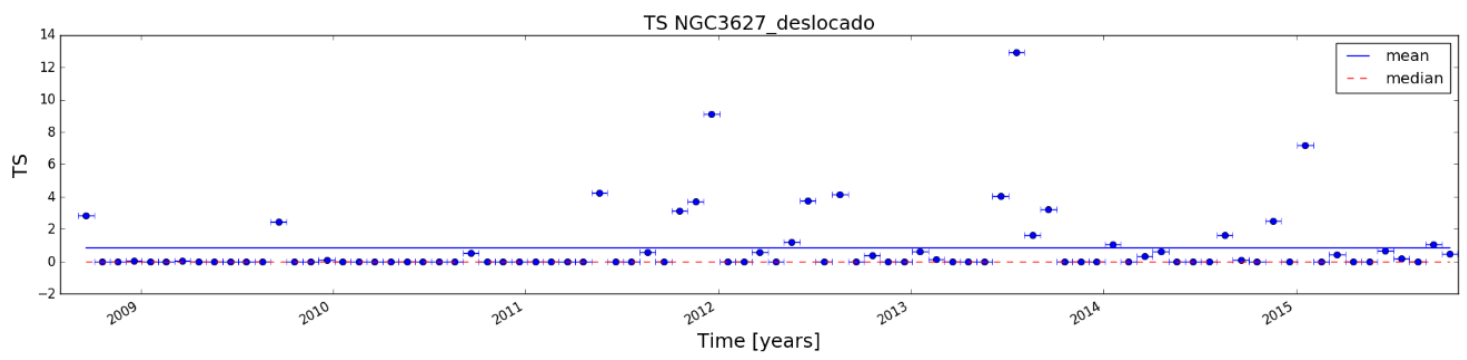

Figura 3.17: TS ao longo do tempo do sinal observado no campo das NGCs 3627 e 3623. Em nenhum momento a atividade do alvo esteve acima de $4 \sigma$.

\subsection{Periodogramas}

Nesta seção usaremos as técnicas de análise de periodicidades explicadas no capítulo 2, levando em consideração, agora, os efeitos de borda introduzidos na CWT quando temos uma série temporal limitada.

Ao procurarmos por periodicidades em curvas de luz geradas a partir dos dados do Fermi-LAT, é importante ficarmos atentos às possíveis periodicidades espúrias listadas a seguir 7 :

- 96 minutos: período orbital do telescópio.

- 3,2 horas: período padrão para que o Fermi-LAT complete um mapa do céu inteiro (equivalente a duas órbitas do satélite).

- 27,3 dias e/ou seus harmônicos: a Lua é uma forte emissora de raios $\gamma$. Se uma curva de luz for obtida para uma fonte próxima do caminho da Lua no céu, então a emissão lunar pode afetar esta curva de luz e seu espectro de potência mostrará modulações periódicas em 27,3 dias e, possivelmente, em seus harmônicos de alta ordem $(5,46,4,55,3,90$ e 3,03 dias $)$.

- 53,4 dias: período de precessão da órbita do telescópio.

\footnotetext{
${ }^{7}$ Temporal Effects of the LAT Observing Strategy: http://fermi.gsfc.nasa.gov/ssc/data/ analysis/LAT_caveats_temporal.html. Acesso em 20/07/2016.
} 
- 1 ano e/ou seus harmônicos: para curvas de luz obtidas de fontes nas proximidades do caminho percorrido pelo Sol no céu, esperamos observar uma periodicidade em torno de um ano devido aos raios $\gamma$ solares. Num espectro de potência, harmônicos deste período também podem ser detectados.

Nas subseções a seguir, trataremos apenas das fontes PG 1553+113 e NGC 1275, pois foram as únicas analisadas que mostraram comportamento periódico em suas curvas de luz.

\subsubsection{PG $1553+113$}

A análise da periodicidade na curva de luz do PG1553+113 mostrada nas figuras 2.9. 2.10 e 2.12 sofre uma pequena mudança quando levamos em consideração os efeitos de borda na CWT. No caso anterior, o sinal foi considerado cíclico, negligenciando, assim, os efeitos causados pelas bordas da série temporal finita (7 anos). No entanto, levando em conta estes efeitos, uma informação extra aparece: o período aumenta sutilmente com o passar do tempo (figura 3.18 - neste caso, aumentamos o intervalo de tempo analisado para 7 anos e 10 meses).

Esta informação faz com que uma das hipóteses sugeridas para explicar este sinal periódico, de que fosse originado por um binário de SMBH (Ackermann et al., 2015b) introduzindo perturbações periódicas no disco de acreção ou precessionando o jato, seja menos provável. Num binário de SMBH, esperamos que seu período orbital diminua com o tempo, aumentando a frequência de suas possíveis perturbações periódicas, ao contrário do que foi observado.

\subsubsection{NGC 1275}

Durante os sete primeiros anos de observações com o Fermi-LAT, detectamos uma possível periodicidade $(\approx 570$ dias $)$ em raios $\gamma$ no alvo NGC 1275 . Para garantir a robusteza desta análise, resolvemos fazê-la duas vezes usando códigos distintos. Usamos nossa pipeline de produção de curvas de luz (KHH) que, de maneira geral, reproduziu bem os resultados para fontes já conhecidas na literatura (Capítulo 2); e o Enrico 8 , um código independente de análise de dados do Fermi. Usamos também dois métodos distintos de criação de curvas de luz: likelihood analysis e fotometria de abertura. A análise completa da periodicidade em raios $\gamma$ observada nesta fonte é mostrada abaixo com as duas pipelines. Em todos os casos, é notável o sinal em $P \approx 570$ dias.

\footnotetext{
${ }^{8}$ Enrico: https://enrico.readthedocs.io/en/latest/. Acesso em 20/07/2016.
} 

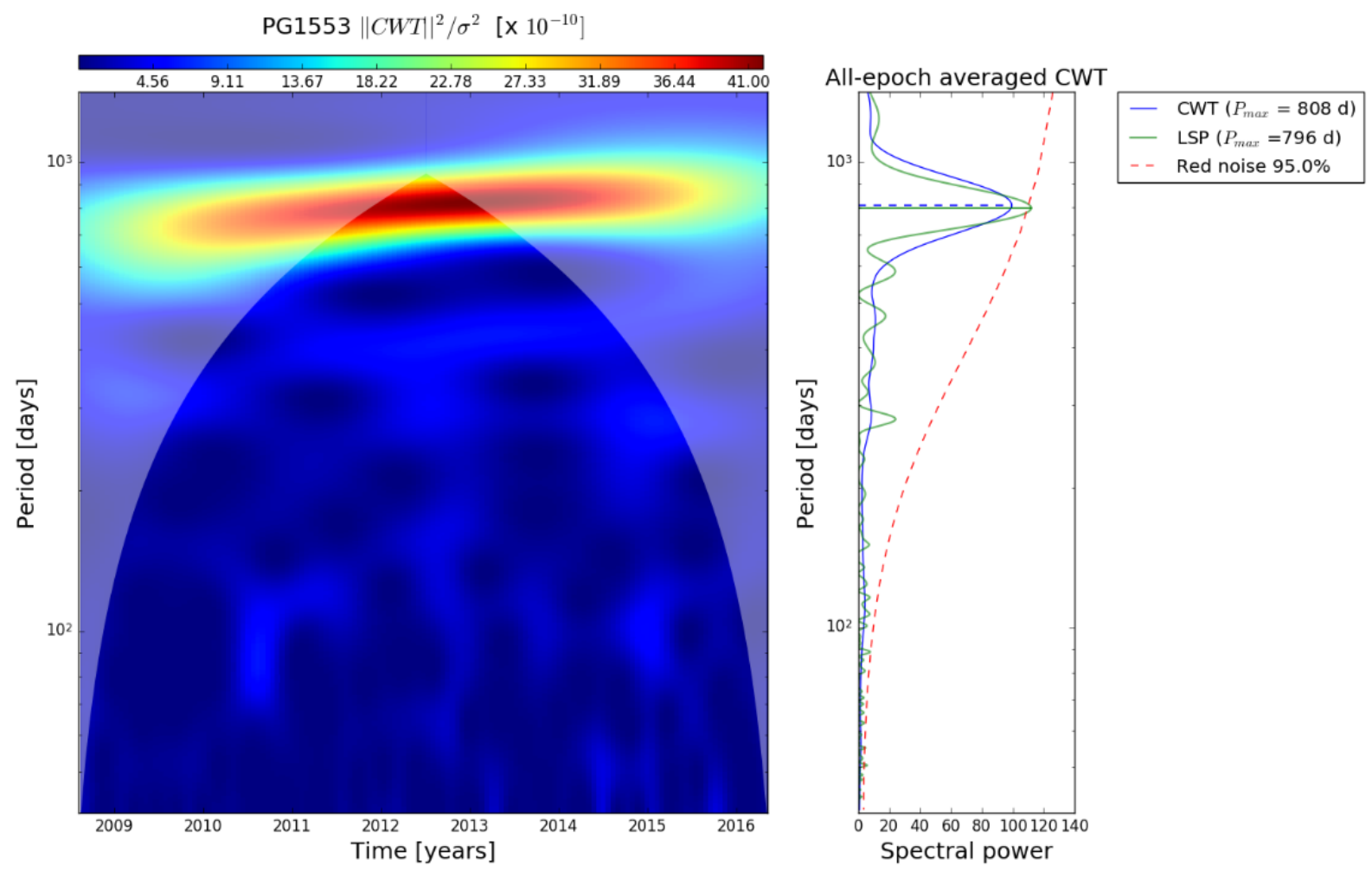

Figura 3.18: CWT do PG1553+113 cobrindo um intervalo de tempo de sete anos e dez meses. É notável, apesar de sutil, o aumento do período com o passar do tempo. A região esbranquiçada representa o cone de influência (COI - cone of influence), onde os efeitos de borda amortecem consideravelmente o sinal. É convencional, quando usamos o wavelet de Morlet, definir a largura do COI a partir das bordas como $l=\sqrt{2} P$, onde $\mathrm{P}$ é o valor do período no eixo y. 


\section{KHH}

A CWT baseada na curva de luz com bins de 10 dias obtida com o KHH (figura 3.2 mostra um pico em torno de 570 dias abaixo do nível de significância (figura 3.19). Veremos nos tópicos seguintes, porém, que a significância deste sinal aumenta à medida que melhoramos nossa resolução temporal.

Os outros sinais com alta intensidade no mapa de wavelets (sinais com periodicidade em torno de 100 dias em 2014 e no final de 2015) da figura 3.19 também serão melhor discutidos no próximo tópico, onde teremos maior resolução temporal.
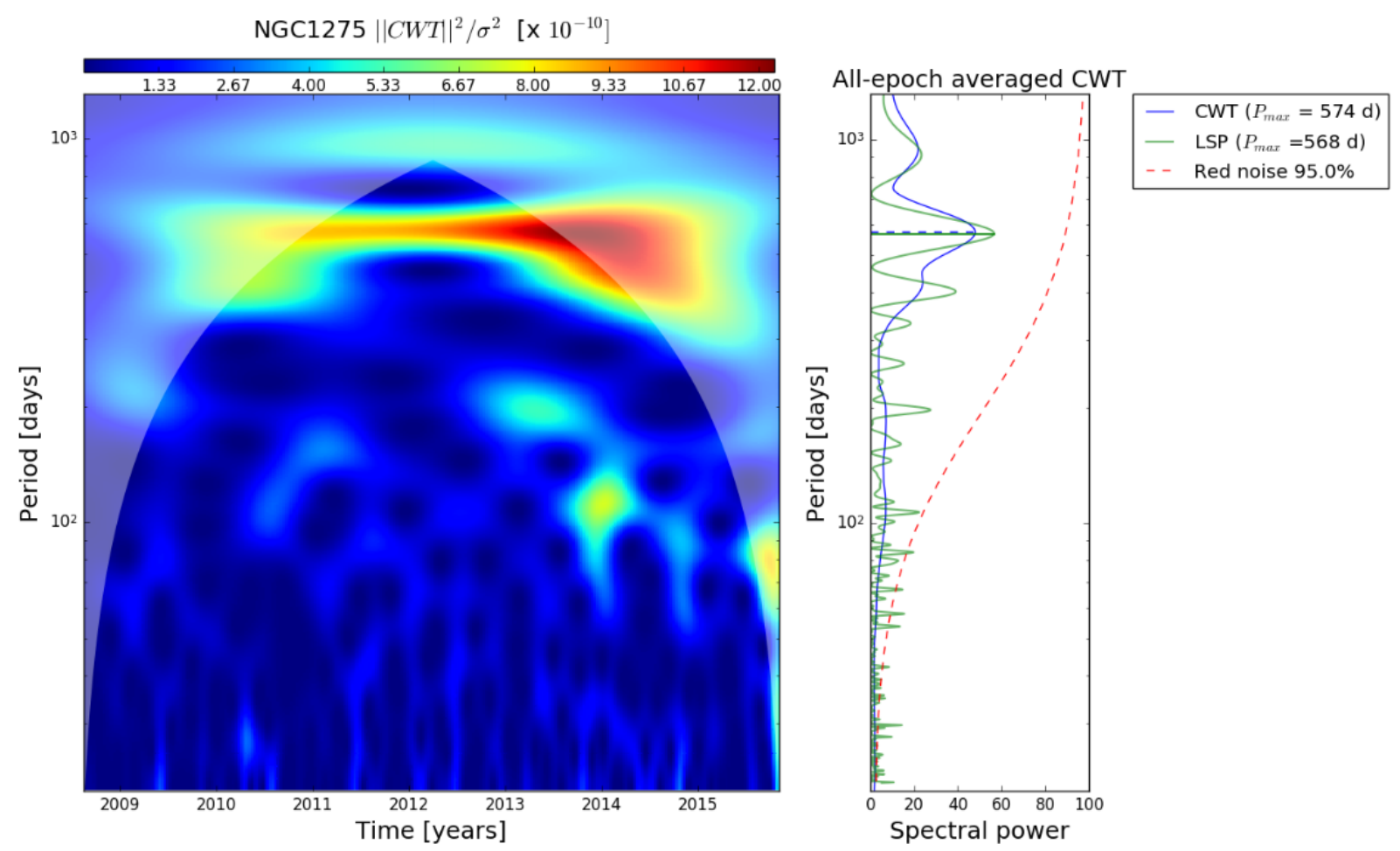

Figura 3.19: CWT da galáxia NGC 1275. A periodicidade em torno de 570 dias é notável, porém possui baixa significância. Os pequenos picos significativos em altas frequências correspondem aos flares na curva de luz da figura 3.2 (mal)interpretados como frequências estacionárias pela análise de Lomb-Scargle. Este problema não aparece na média temporal da CWT (linha azul no gráfico da direita).

A periodicidade que estamos procurando na figura 3.2 não é óbvia de ser encontrada à primeira vista, mas, ao suavizarmos a curva de luz (no caso, escolhendo bins temporais maiores), seus picos tornam-se bem mais evidentes, como indicado pelas barras pretas na figura 3.20. Notamos que no intervalo entre 2011 e 2013 não aparece nenhum pico óbvio. 


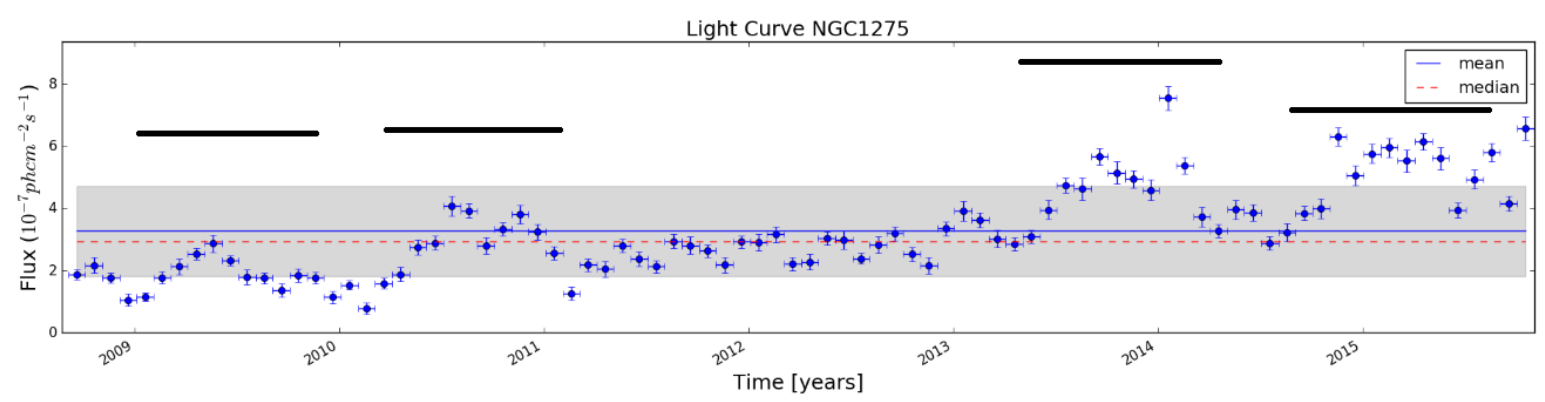

Figura 3.20: curva de luz do alvo NGC 1275 com bins temporais mensais. Os picos referentes a uma periodicidade de $\sim 570$ dias apontados no mapa de wavelets da figura 3.19 são, agora, mais facilmente notados.

\section{Enrico}

Adotando bins temporais de 1 dia, refizemos a análise usando o Enrico. Por escolhermos um bin tão pequeno, ganhamos muito em resolução temporal, mas acabamos diminuindo consideravelmente o sinal/ruído em cada uma das medidas (figura 3.21).

Importando os resultados da curva de luz obtida com o Enrico para o KHH, pudemos analisar seu comportamento periódico (figuras 3.22 e 3.23 ). O pico em torno de 570 dias agora aparece de maneira muito mais significativa. Notamos ainda a presença de vários picos menores de alta significância, discutidos em mais detalhes na figura 3.22

É importante notarmos que, enventualmente, flares aparecem como "periodicidades transientes" num mapa de wavelets. O quadrado branco nas figuras 3.21 e 3.22 dá destaque aos pequenos flares que foram apontados como uma periodicidade passageira na análise por wavelets.

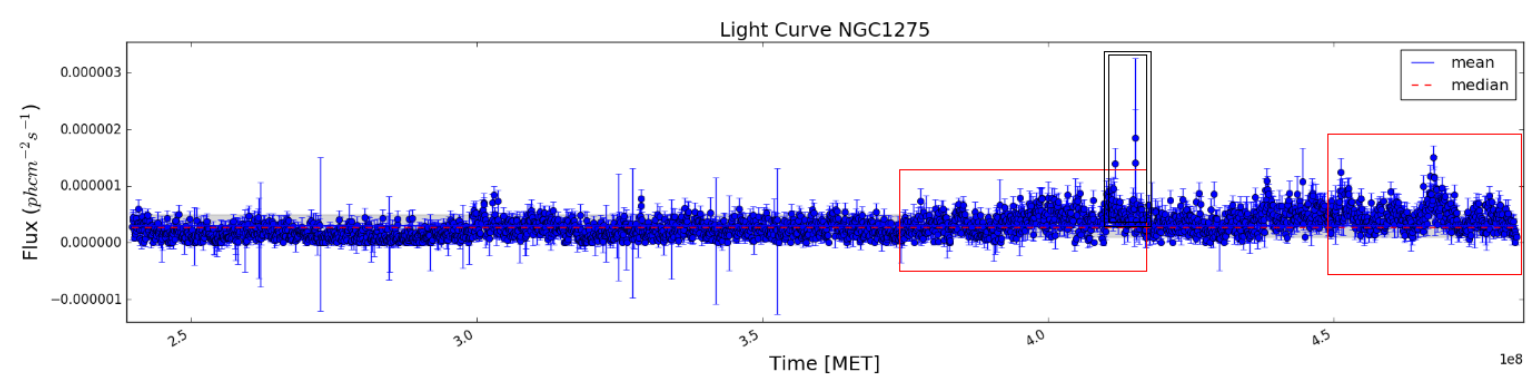

Figura 3.21: curva de luz com bins de 1 dia ao longo de 7 anos da NGC 1275. Para a criação desta curva de luz fixamos o índice espectral em $\gamma=2,07$. A alta resolução temporal permite uma melhor análise por FFT, Lomb-Scargle e wavelets, mas diminui a precisão na medida do fluxo em cada bin. 

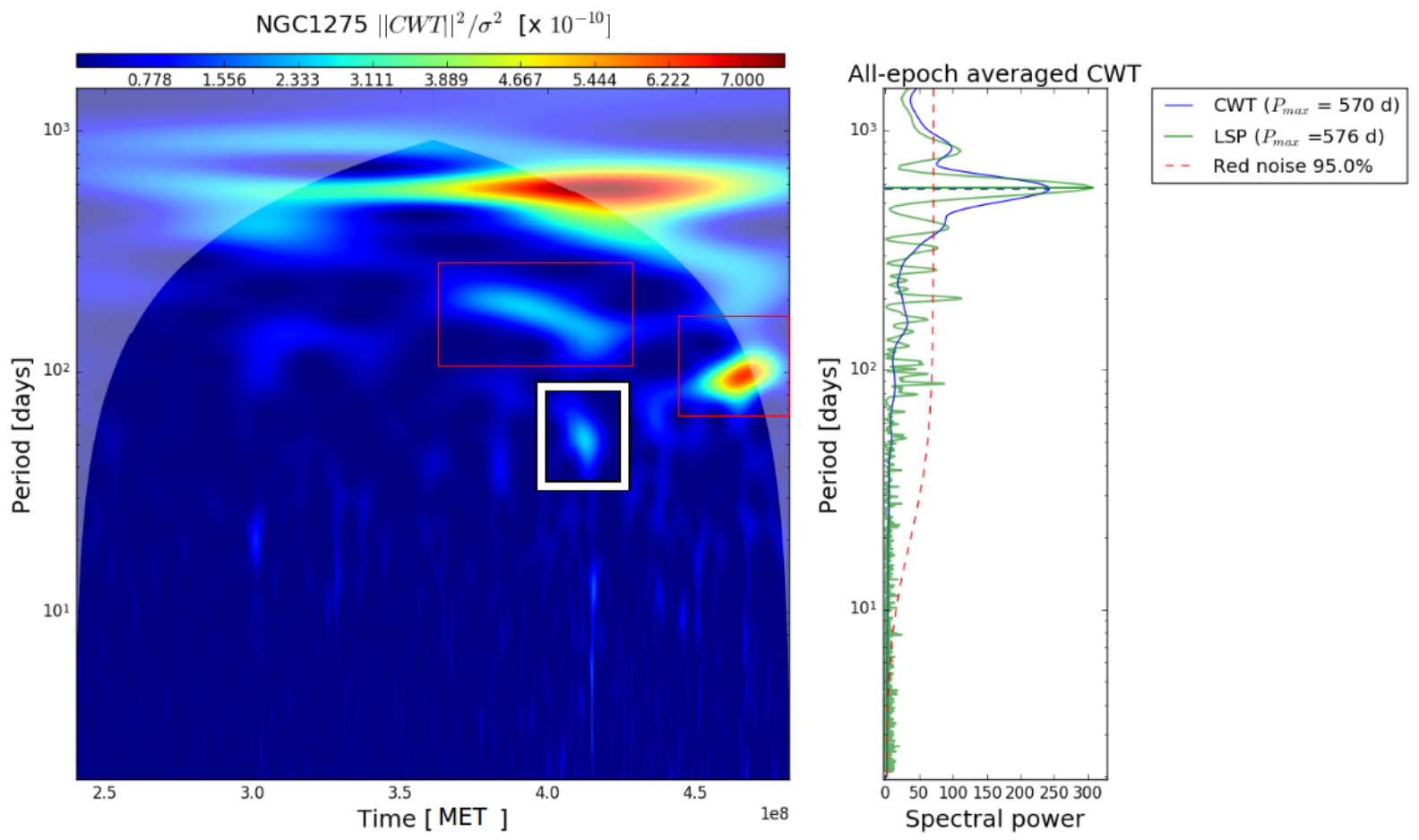

Figura 3.22: CWT da galáxia NGC 1275. A periodicidade em 570 dias é evidente e atinge mais de $95 \%$ de confiança no periodograma de Lomb-Scargle e na média da CWT. Os picos secundários em torno de 90 e 200 dias correspondem às periodicidades transientes destacadas com quadrados vermelhos na figura 3.21. Uma nova análise com bins temporais de dois dias (maior sinal/ruído) será feita nestas regiões assim que possível.

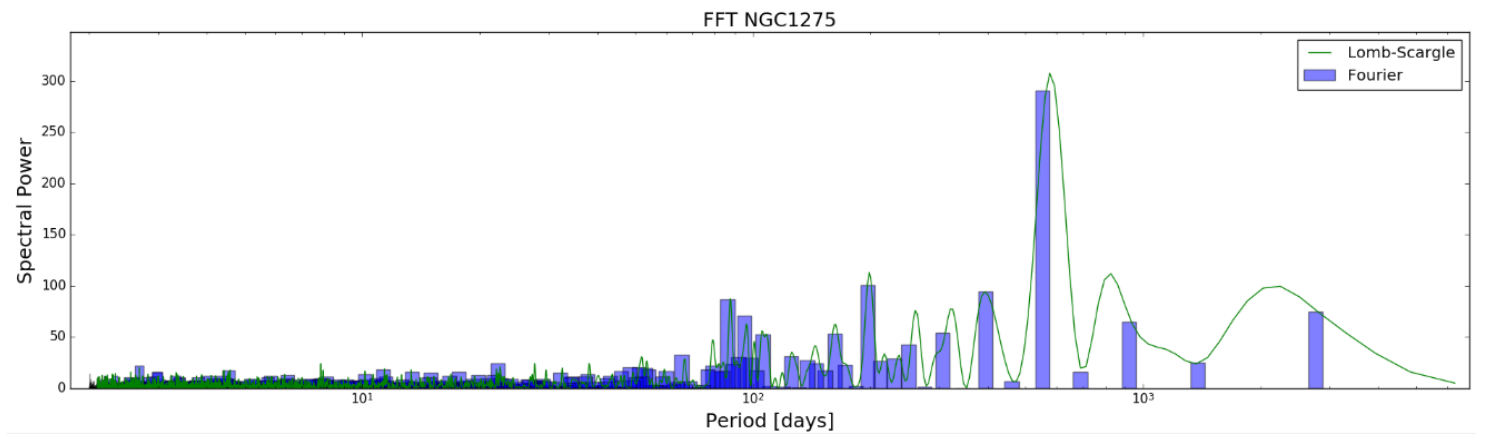

Figura 3.23: análise de Fourier e periodograma de Lomb-Scargle indicando um período em torno de 570 dias. 


\section{Fotometria de abertura}

Para a fotometria de abertura, selecionamos uma ROI com apenas $1^{\circ}$ de raio em torno do alvo. Uma ROI pequena deve excluir a maioria dos eventos de fundo, que, neste caso, não podem ser modelados 9 , e focar somente nos eventos que são mais prováveis de ter origem na nossa fonte.

A curva de luz gerada por fotometria de abertura para a galáxia NGC 1275 (figura 3.24 ) continua indicando uma periodicidade em torno de 570 dias (figuras 3.25 e 3.26). Por conta de problemas numéricos, desta vez não foi possível a construção de um periodograma de Lomb-Scargle.

As altas frequências detectadas pela FFT (quadrados vermelhos na figura 3.25) são falsas e estão relacionadas a dois períodos de flare, um em 2010 e o outro em 2014 (quadrados vermelhos nas figuras 3.24 e 3.26 ).

Ainda podemos observar, na figura 3.26, uma periodicidade em torno de 90 dias no final do ano de 2015, consistente com um dos picos da análise de Lomb-Scargle nos dados do Enrico (tópico anterior), porém com baixa significância quando tomamos a média da CWT no tempo.

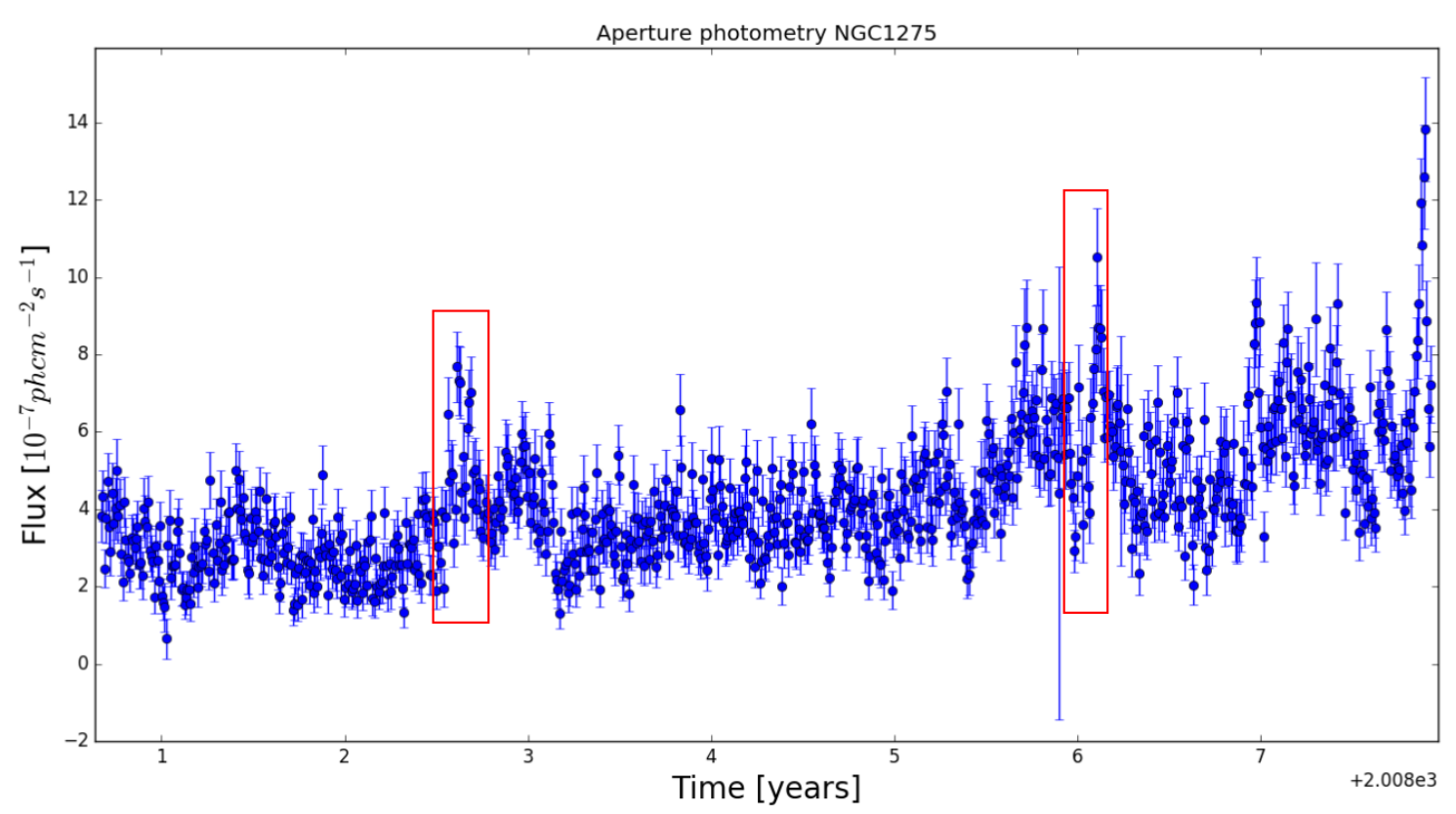

Figura 3.24: fotometria de abertura da NGC 1275 com bins de três dias. Este método é menos rigoroso que a fotometria por likelihood analysis, onde a emissão de background e foreground podem ser modeladas.

${ }^{9}$ Fermi LAT Aperture Photometry: http://fermi.gsfc.nasa.gov/ssc/data/analysis/scitools/ aperture_photometry.html. Acesso em 21/07/2016. 


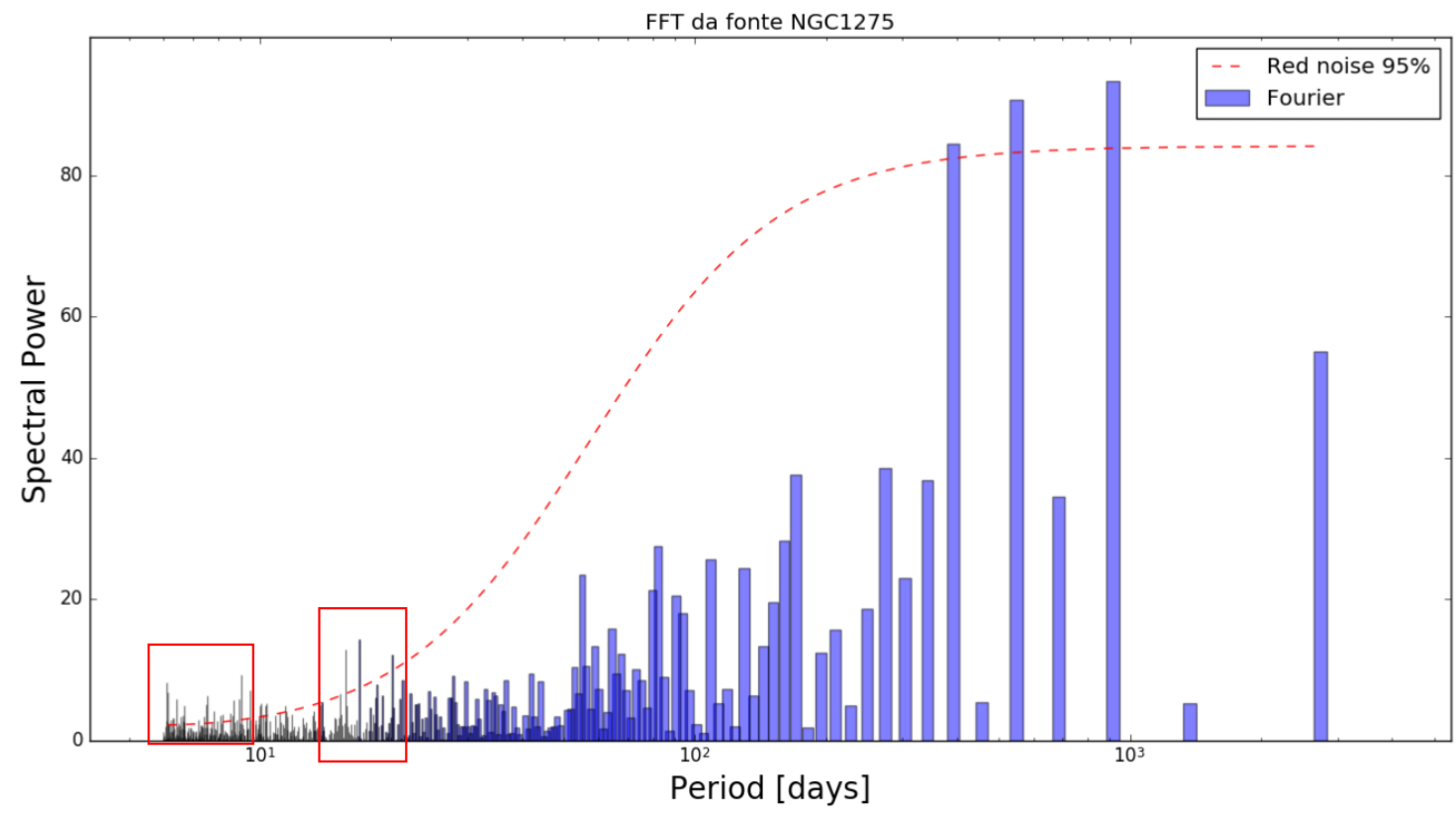

Figura 3.25: transformada de Fourier do sinal da NGC 1275 medido por fotometria de abertura, com vários picos de alta significância, sendo os mais notáveis $P_{1} \approx 395, P_{2} \approx 557$ e $P_{3} \approx 928$ dias. Dois destes picos, $P_{1}$ e $P_{3}$, estão associados a periodicidades transientes e aparecem de maneira sutil em todos os mapas de wavelets que fizemos para a NGC 1275 (figuras 3.19, 3.22 e 3.26). Os picos em altas frequências (caixas vermelhas) estão associados a períodos de flare na curva de luz da figura 3.24 interpretados erroneamente como frequências estacionárias pela FFT. 

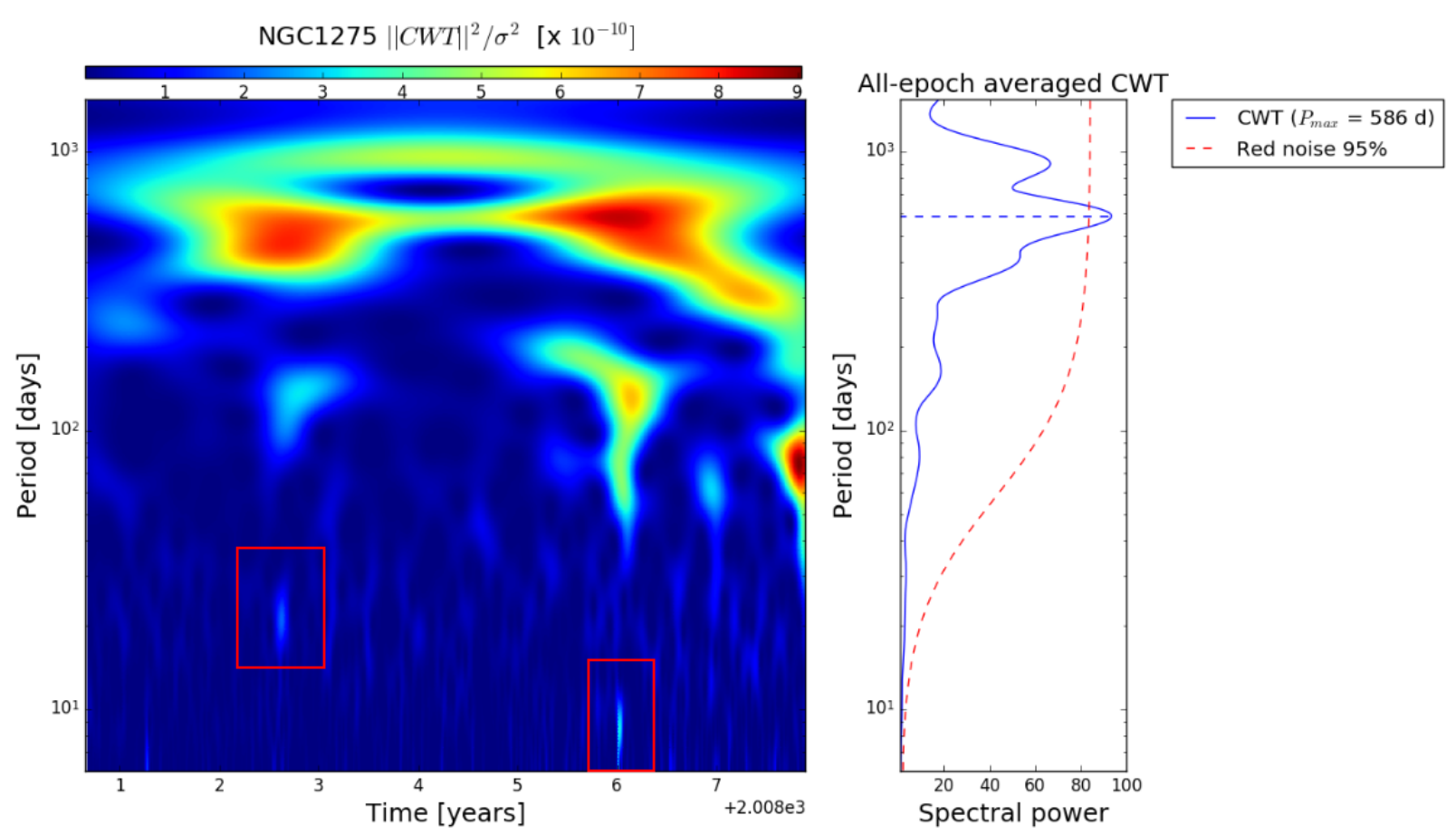

Figura 3.26: CWT da NGC 1275 baseada em sua curva de luz por fotometria de abertura, com um único pico significativo em $P \approx 586$ dias. A COI não pode ser graficada por conta de problemas numéricos, mas seus efeitos foram levados em conta. As regiões envolvidas pelos quadrados vermelhos representam flares na nossa curva de luz (figura 3.24) e não devem ser confundidas com periodicidades transientes.

\subsection{Evidência de novas fontes de raios $\gamma$}

Detectamos, por acaso, três sinais muito intensos em raios $\gamma$ ao analisarmos os mapas de resíduos ou de TS dos nossos alvos (figura 3.27). As três fontes possuem alta significância estatística $(T S>100)$ mas não estão catalogadas no último catálogo de fontes do Fermi, o 3FGL (Acero et al., 2015b), que abrange os primeiros quatro anos de aquisição de dados do telescópio. Isto sugere que estas fontes entraram em período de alta atividade após a construção do catálogo. Esta hipótese é comprovada pelas figuras 3.28, 3.29 e 3.31.

Como a significância estatística destas fontes é praticamente nula durante a mior parte do tempo, fixamos seus índices espectrais (que não variam muito durante os períodos de alta atividade, como podemos observar nas figuras 3.28, 3.29 e 3.31) no melhor valor estimado pela binned likelihood em todo o intervalo de sete anos de dados.

Além das posições, índices espectrais, curvas de luz e curvas de TS, obtivemos as SEDs (entre $100 \mathrm{MeV}$ e $300 \mathrm{GeV}$ ) de duas destas fontes usando o Enriç 10 . Uma análise em outros comprimentos de onda $(<100 \mathrm{MeV})$, com melhor resolução espacial e acesso aos valores da SED em baixas energias, é essencial para melhor compreendermos e até identificarmos estas fontes.

Ainda não associamos estes sinais em raios $\gamma$ com fontes em comprimentos de onda

\footnotetext{
${ }^{10}$ Enrico: http://enrico.readthedocs.io/en/latest/index.html. Acesso em 23/07/2016.
} 
menos energéticos e, portanto, ainda não temos informação sobre seus redshifts. Para melhor diferenciar estas fontes, resolvemos chamá-las de Cangaceira, Rapadura e Mandacaru 11 ,

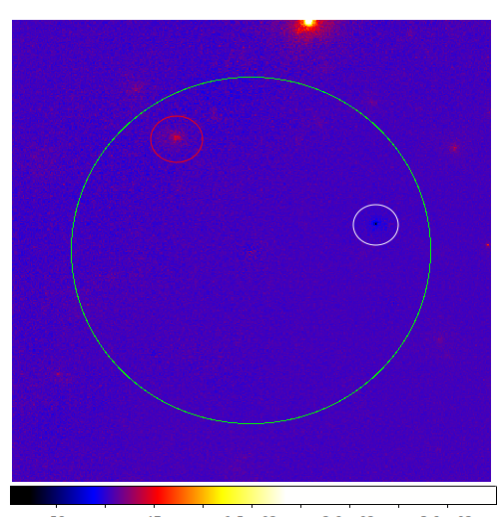

(a) Cangaceira. Mapa de resíduos.

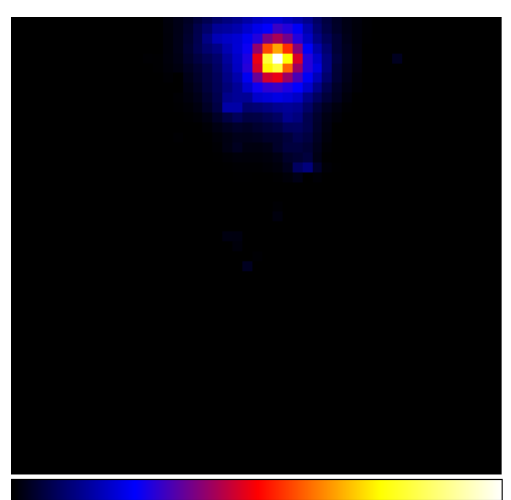

$\begin{array}{lllllllll}18 & 35 & 53 & 70 & 88 & 106 & 123 & 141 & 159\end{array}$

(b) Mandacaru. TS de resíduos.

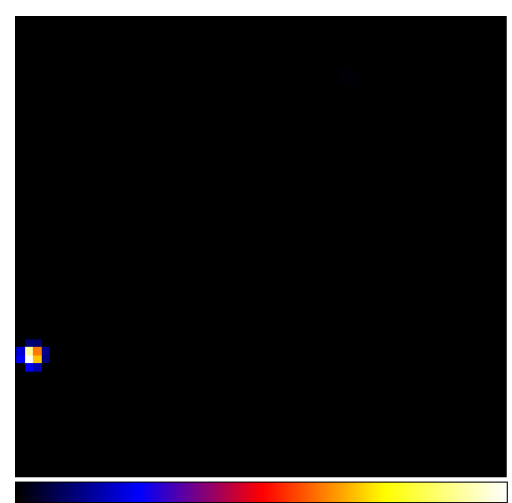

$\begin{array}{lllllllll}22 & 45 & 67 & 90 & 112 & 135 & 157 & 180 & 202\end{array}$

(c) Rapadura. TS de resíduos.

Figura 3.27: esquerda: mapa de resíduos (contagens - modelo) centrado na NGC 1275. O círculo verde, com $15^{\circ}$ de raio, indica a região do céu onde todas as fontes catalogadas no 3FGL foram subtraídas. O círculo vermelho envolve um forte sinal em raios $\gamma$ não catalogado, enquanto que o círculo branco indica a posição de uma fonte cujo fluxo foi superestimado. Centro e direita: mapas de TS com campo de $10^{\circ} \times 10^{\circ}$ e $0,2^{\circ}$ por pixel centrados nos alvos NGC 1055 (centro) e NGC 5371 (direita). Nossos alvos sequer aparecem no mapa, em contraste com as novas fontes nas bordas dos mapas.

\section{Cangaceira}

Posicionada em $R A \approx 59,87^{\circ}, D e c \approx 50,97^{\circ}$ (J2000.0, Equatorial), esta fonte foi encontrada ao fazermos o mapa de resíduos do campo da NGC 1275 (figura 3.27a). Para uma melhor convergência da curva de luz, fixamos o valor do índice espectral em 2,57, que foi o melhor valor estimado pela binned likelihood para esta fonte usando sete anos de dados. Dentre as três fontes analisadas nesta seção, a Cangaceira foi a única para a qual ainda não construímos uma SED (será feita assim que possível).

Se considerarmos que esta fonte está no Universo local $(z \approx 0)$, a equação 3.1 permite que estimemos o tamanho da região emissora de raios $\gamma$ em $d<60$ dias-luz. A figura 3.28 resume nossos resultados.

\section{Rapadura}

Posicionada em $R A \approx 215,68^{\circ}, D e c \approx 37,62^{\circ}$ (J2000.0, Equatorial), esta fonte foi encontrada ao fazermos o mapa de TS do campo da NGC 5371 (figura 3.27c). Para uma melhor convergência da curva de luz, forçamos um valor fixo de 2,00 para o índice

\footnotetext{
${ }^{11}$ Inspirado em termos da cultura nordestina.
} 


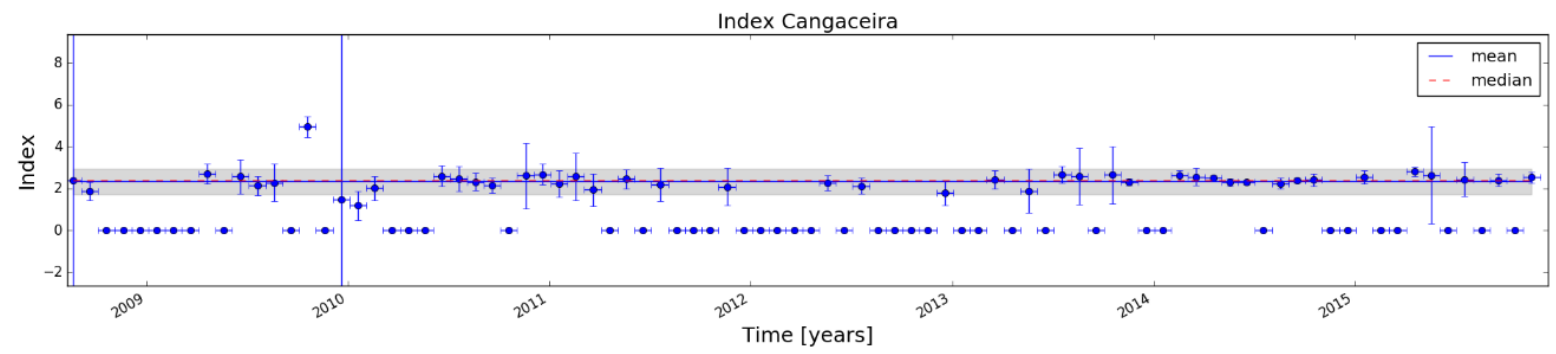

(a) Índice espectral.

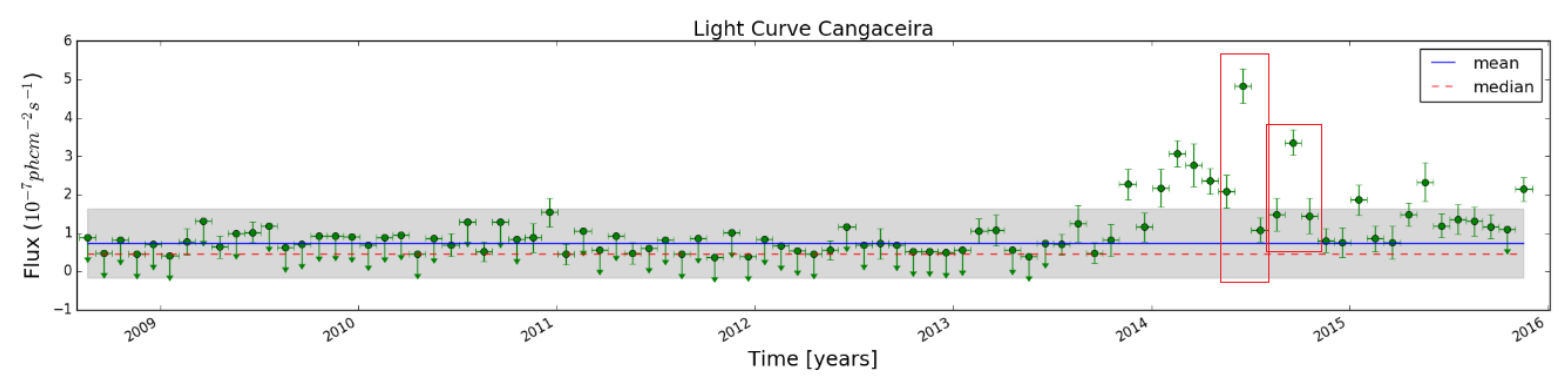

(b) Curva de luz.

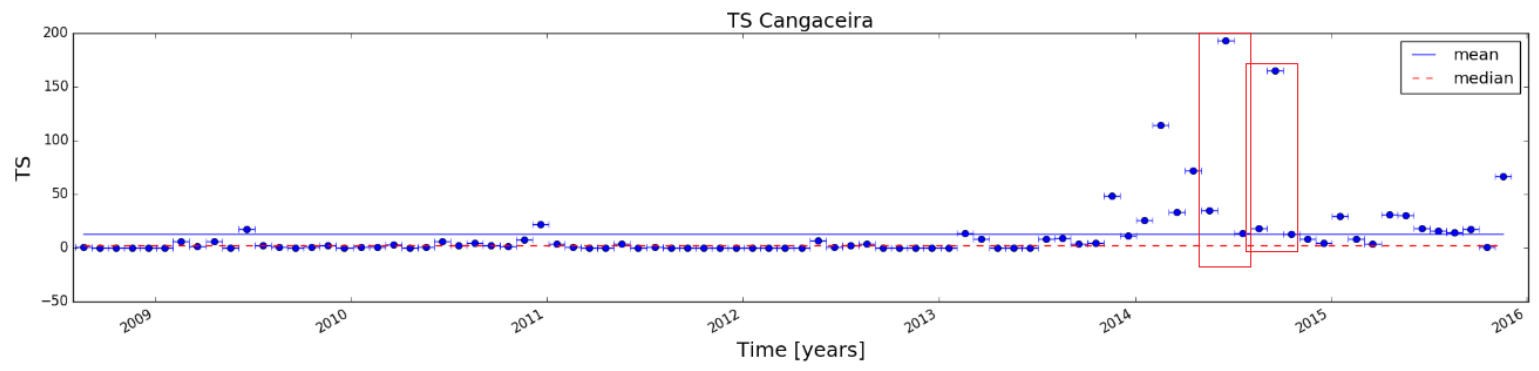

(c) Curva de TS.

Figura 3.28: variabilidade mensal do índice espectral (a), fluxo (b) e TS (c) da fonte Cangaceira. Para obtermos melhor convergência do sinal, a curva de luz (b) foi gerada mantendo-se o índice espectral (a) fixo em 2,57 (melhor valor estimado pela binned likelihood para esta fonte nos sete anos de dados analisados). Notamos que esta fonte permaneceu inativa durante os primeiros anos de observações com o Fermi, não sendo, assim, catalogada no 3FGL. Observamos ainda alguns flares (caixas vermelhas) com alta significância estatística (até $\sqrt{T S} \sim 14 \sigma$ ) que nos permitem afirmar que esta fonte varia bruscamente numa escala de tempo de 30 dias. Caso esta fonte encontre-se no Universo local $(z \approx 0)$, a região emissora de raios $\gamma$ que observamos deve ser menor que $\sim 60$ dias-luz.

espectral, que foi o melhor valor estimado pela binned likelihood nos sete anos de dados. A figura 3.29 resume nossos resultados.

Para entendermos melhor a origem física da emissão em raios $\gamma$ desta fonte, é essencial extraírmos informação de sua SED. Considerando apenas o período de alta atividade, entre 15-09-2013 e 15-08-2014, modelamos a SED desta fonte com uma lei de potência (equação 2.1). A figura 3.30 mostra a SED e o mapa de contagens da fonte Rapadura. Esta SED possibilita um cenário de emissão de raios $\gamma$ por SSC, característico de blazares, no entanto, para uma melhor modelagem da SED, precisamos de dados em outros 


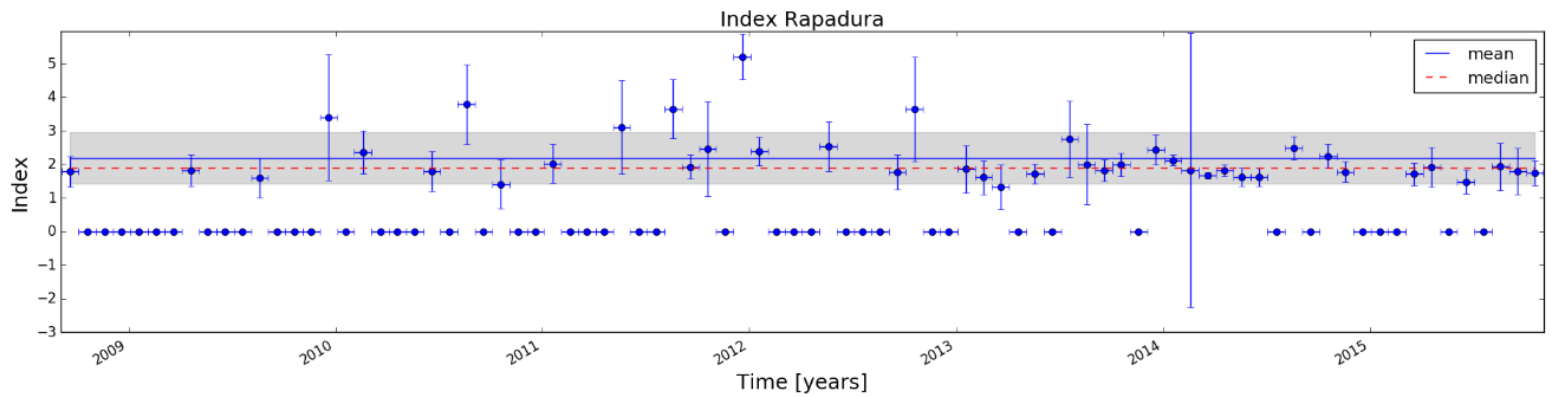

(a) Índice espectral.

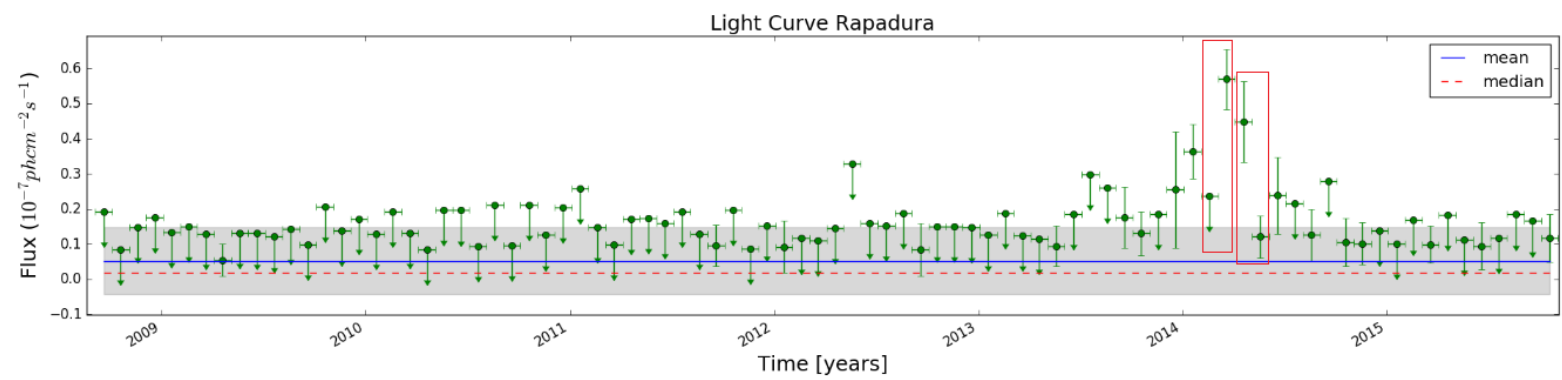

(b) Curva de luz.

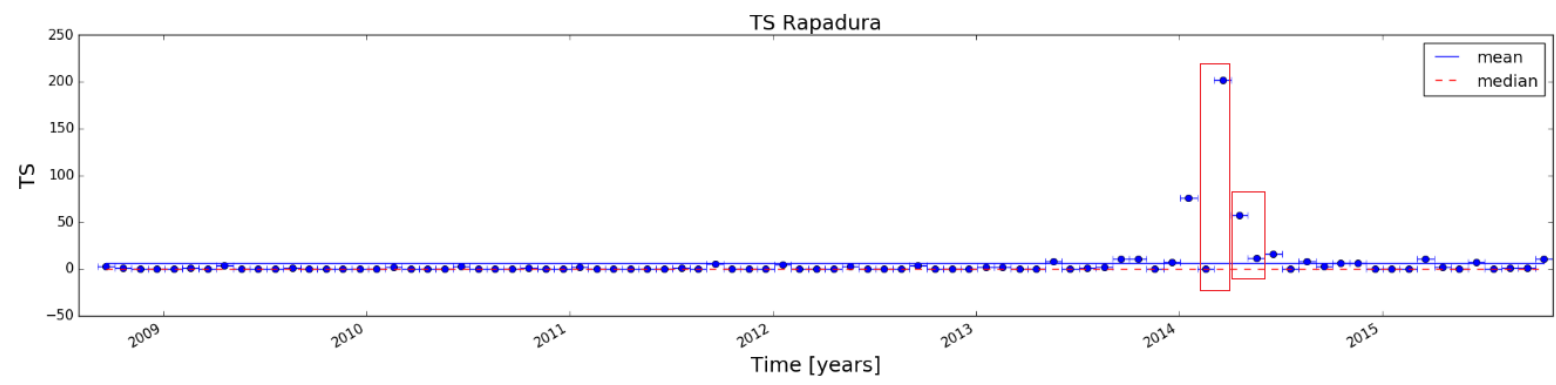

(c) Curva de TS.

Figura 3.29: variabilidade mensal do índice espectral (a), fluxo (b) e TS (c) da fonte Rapadura. Para obtermos melhor convergência do sinal, a curva de luz (b) foi gerada mantendo-se o índice espectral (a) fixo em 2,00 (melhor valor estimado pela binned likelihood para esta fonte nos sete anos de dados analisados). Notamos que, assim como a Cangaceira (figura 3.28), esta fonte permaneceu inativa durante os primeiros anos de observações com o Fermi, não sendo, assim, catalogada no 3FGL. Observamos ainda dois flares (caixas vermelhas) com alta significância estatística (até $\sqrt{T S} \sim 14 \sigma$ ) que nos permitem afirmar que esta fonte varia bruscamente numa escala de tempo de 30 dias. Caso esta fonte encontre-se no Universo local $(z \approx 0)$, sua região emissora de raios $\gamma$ deve ser menor que $\sim 60$ dias-luz (equação 3.1).

comprimentos de onda.

\section{Mandacaru}

Posicionada em $R A \approx 39,93^{\circ}, D e c \approx 4,34^{\circ}$ (J2000.0, Equatorial), esta fonte foi encontrada ao fazermos o mapa de TS do campo da NGC 1055 (figura 3.27b). Para uma melhor convergência da curva de luz, fixamos o valor do índice espectral em 2,15, que 

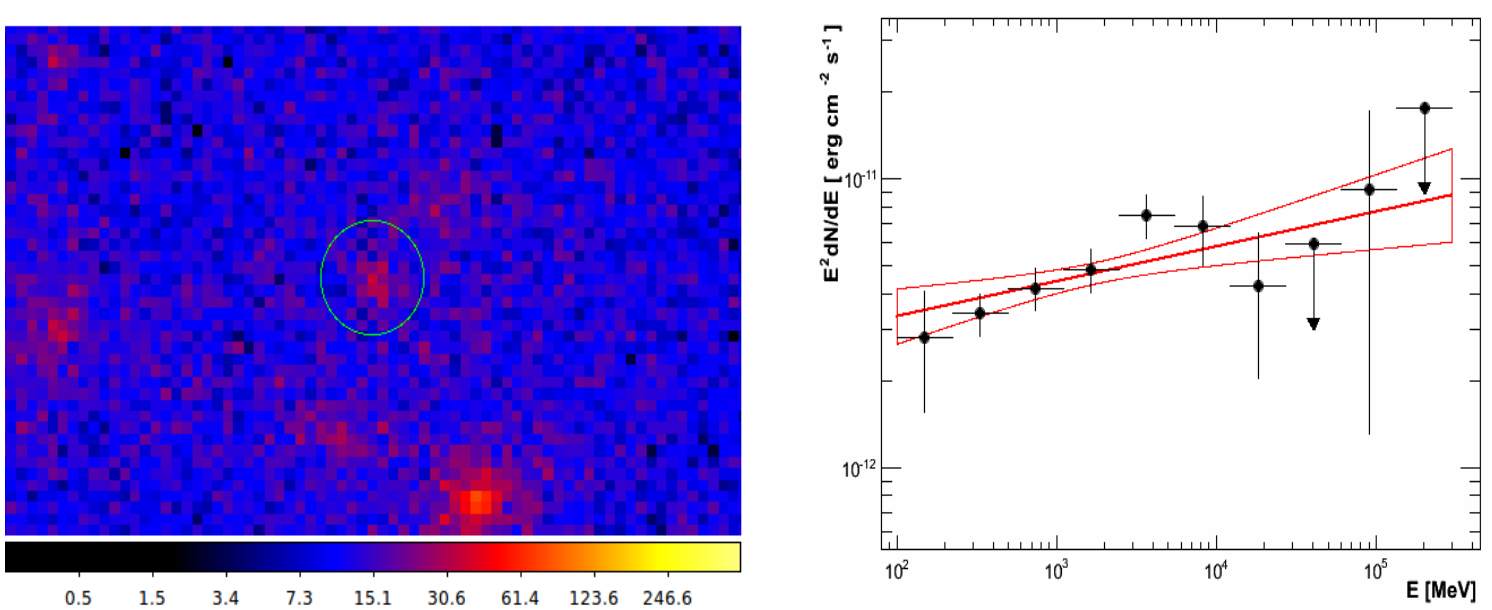

Figura 3.30: esquerda: mapa de contagens da fonte Rapadura com base em sete anos de observações. A fonte encontra-se dentro do círculo verde. Direita: SED da fonte modelada com uma lei de potência. As linhas vermelhas mais finas são os contornos da região com $1 \sigma$ de confiança. Os limites superiores (indicados por setas) foram calculados nos bins de energia onde a significância estatística foi menor que $3 \sigma$.

foi o melhor valor estimado pela binned likelihood nos sete anos de dados. A figura 3.31 resume nossos resultados.

Observamos a evolução da SED desta fonte durante seu período de alta atividade e percebemos que, durante os dois meses de maior atividade (de 01-06-2014 até 30-07-2014), sua SED não evoluiu significativamente, indicando uma emissão forte de raios $\gamma$ apenas em baixas energias. A figura 3.32 mostra as SEDs da fonte Mandacaru. O grande número de limites superiores computados para os bins de alta energia dificulta a interpretação desta SED e, para uma melhor modelagem espectral, precisamos de dados em outros comprimentos de onda. 


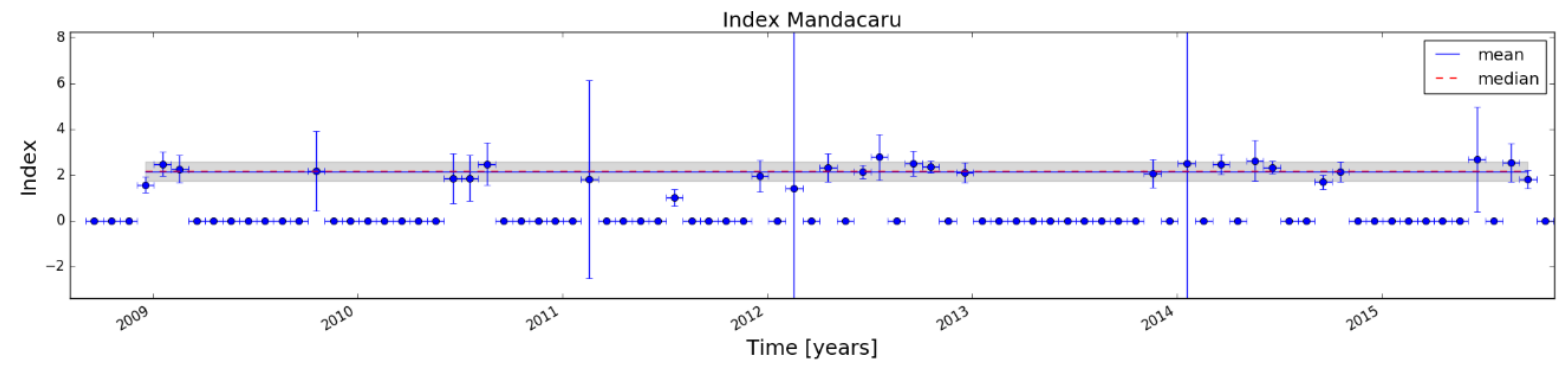

(a) Índice espectral.

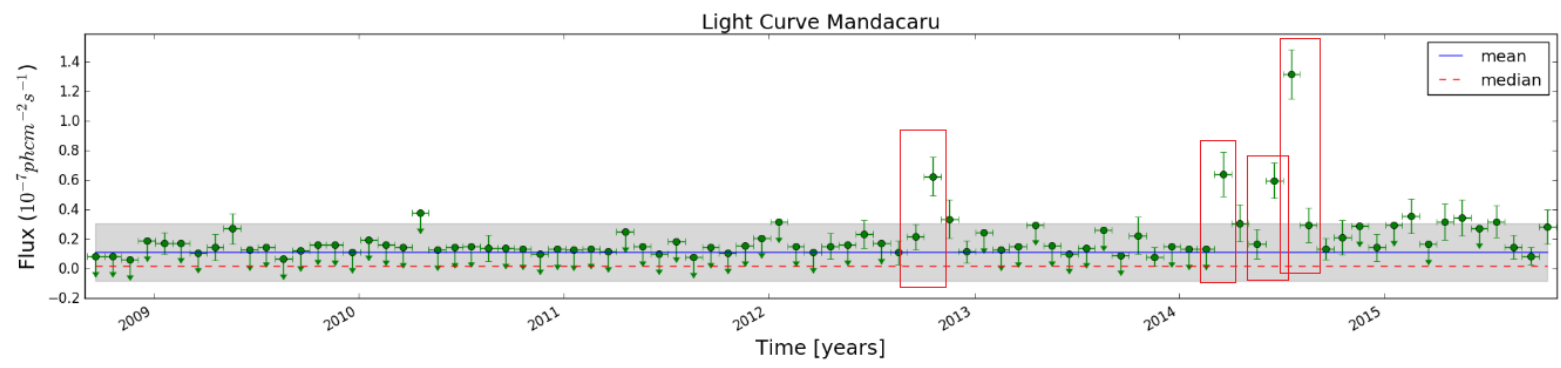

(b) Curva de luz.

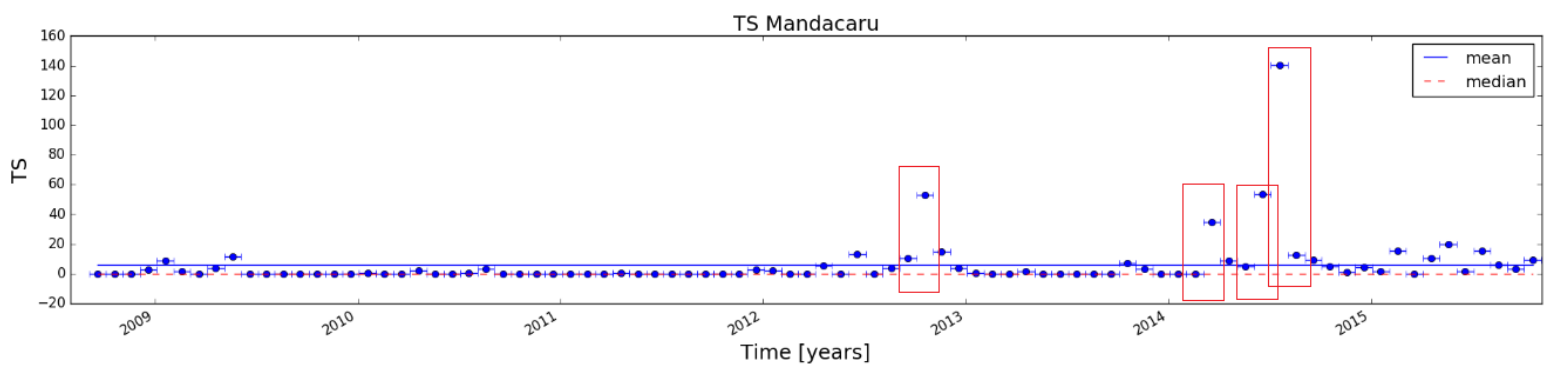

(c) Curva de TS.

Figura 3.31: variabilidade mensal do índice espectral (a), fluxo (b) e TS (c) da fonte Mandacaru. Para obtermos melhor convergência do sinal, a curva de luz (b) foi gerada mantendo-se o índice espectral (a) fixo em 2,15 (melhor valor estimado pela binned likelihood para esta fonte nos sete anos de dados analisados). Notamos que, assim como a Cangaceira e Rapadura (figuras 3.28 e 3.29), esta fonte permaneceu inativa durante os primeiros anos de observações com o Fermi, não sendo, assim, catalogada no 3FGL. Observamos ainda vários flares (caixas vermelhas) com alta significância estatística (até $\sqrt{T S} \sim 12 \sigma$ ) que nos permitem afirmar que esta fonte varia bruscamente numa escala de tempo de 30 dias. Caso esta fonte encontre-se no Universo local $(z \approx 0)$, sua região emissora de raios $\gamma$ deve ser menor que $\sim 60$ dias-luz (equação 3.1). 

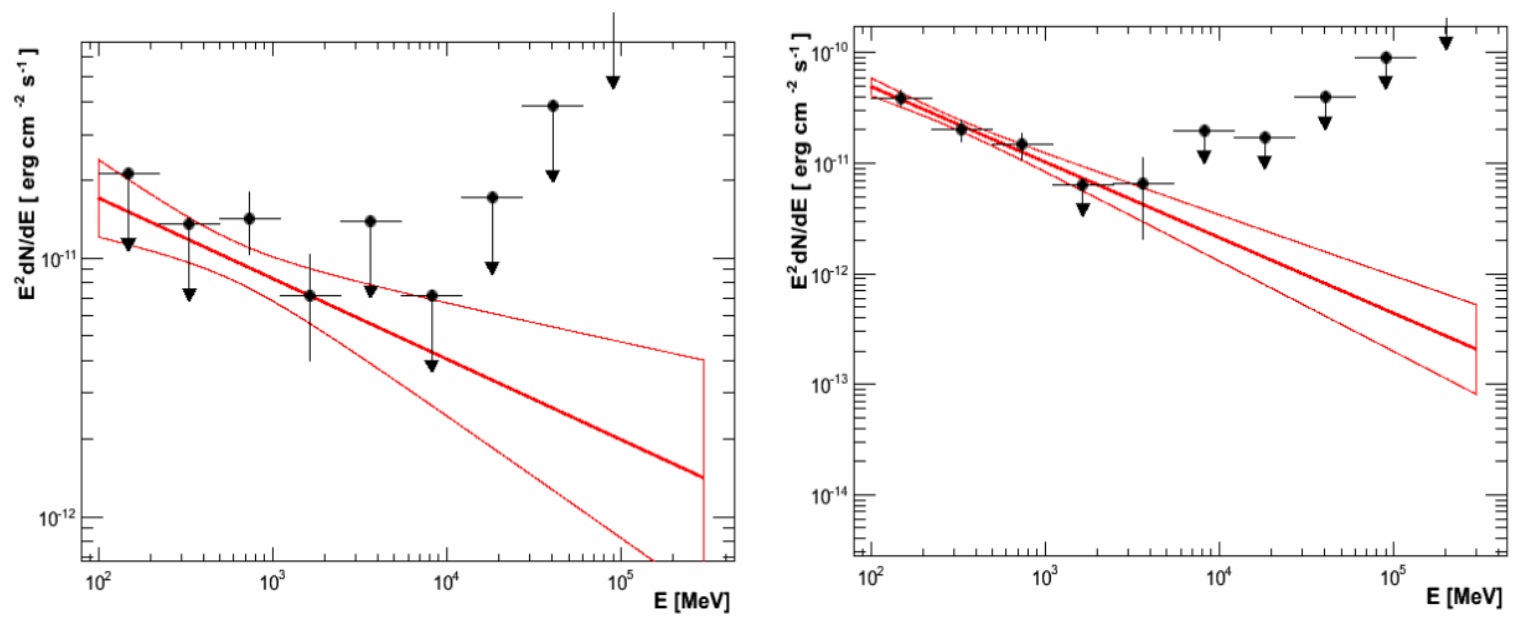

Figura 3.32: SEDs da fonte Mandacaru modelada com uma lei de potência. Esquerda: SED do flare em junho de 2014. Direita: SED do flare que ocorreu em julho de 2014. As linhas vermelhas mais finas são os contornos da região com $1 \sigma$ de confiança. Os limites superiores (indicados por setas) foram calculados nos bins de energia onde a significância estatística foi menor que $3 \sigma$. 
CAPÍTULO 4

\section{Conclusões e perspectivas}

Neste trabalho discutimos os métodos de criação e análise de curvas de luz em raios $\gamma$. Fizemos algumas descobertas, como o crescimento aparentemente linear da periodicidade do blazar PG 1553+113, evidência de periodicidade na Seyfert NGC 1275, evidências de novas fontes de raios $\gamma$ e ainda conseguimos inferir limites superiores para o tamanho da região emissora de raios $\gamma$ de três LLAGNs (NGC 1275, M87 e NGC 3301). Começamos, também (embora ainda não tenhamos chegado a um consenso sobre o assunto), a usar mapas de wavelets como auxiliares na detecção de flares. Nossos resultados estão resumidos nos tópicos a seguir.

\section{LLAGNs}

Conseguimos restringir o tamanho da região emissora de raios $\gamma$ de três LLAGNs: Perseus A, Messier 87 e NGC 3301, cujos tamanhos são, respectivamente, $d \lesssim 504 r_{s}$, $d \lesssim 53 r_{s}$ e $d \lesssim 17080 r_{s}$. Em nenhuma das estimativas fizemos correções pelo fator de Doppler, devido aos efeitos relativísticos de projeção causados pelo (possível) jato. Para uma estimativa mais precisa do tamanho dessas regiões, esta correção deve ser levada em conta, e, de acordo com o ângulo de inclinação do jato em relação à linha de visada, o tamanho da região emissora pode ser consideravelmente maior do que o que estimamos.

Descobrimos também que a maior parte das fontes da nossa amostra não emite raios $\gamma$ o suficiente para serem detectadas pelo Fermi. A maior parte da sua emissão em gama deve vir da interação de raios cósmicos com o ISM, como é o caso da Via Láctea, Andrômeda, SMC e LMC (Massaro et al., 2016), sendo, portanto, muito fracas para serem detectadas pelo Fermi. 


\section{Fontes quasi-periódicas}

Quando consideramos os efeitos de borda na CWT, o período observado no PG1553+133 parece aumentar sutilmente e de maneira linear no intervalo de aproximadamente oito anos. Esta observação torna menos provável um dos cenários descritos na referência Ackermann et al. (2015b), onde é sugerido que a presença de um SMBH binário, no estágio inicial do regime de emissão de ondas gravitacionais, esteja introduzindo perturbações periódicas no disco de acreção ou precessionando o jato do blazar. A curva de luz desta fonte deve continuar sendo monitorada para que obtenhamos melhores resultados.

Até o momento, o blazar PG1553+113 era a única fonte extragaláxtica periódica em raios $\gamma$ já detectada. Nosso trabalho indica que a NGC 1275 é mais uma forte candidata a $\mathrm{QPO}(P \approx 570$ dias), com um nível de confiança que excede 95\%, dependendo do tamanho do bin temporal usado na curva de luz.

\section{Fontes que despertaram}

Três fontes não catalogadas no 3FGL foram observadas emitindo fortemente em raios $\gamma$ por intervalos de tempo relativamente pequenos (da ordem de 1 ano). Esta forte emissão pode ter sido causada pela queda de objetos, como estrelas ou nuvens de gás, num SMBH adormecido (Gillessen et al., 2012), fazendo com que sua emissão em altas energias aumentasse explosivamente. Todas as três fontes apresentam mudanças bruscas no fluxo de raios $\gamma$ no intervalo de tempo de um mês. Observações em outros comprimentos de onda serão fundamentais para descobrirmos a natureza dessas fontes. Uma vez associadas a algum objeto galáctico/extragaláctico, poderemos melhorar a resolução temporal de suas curvas de luz e extrair informações mais precisas sobre que tipo de fenômeno está, de fato, acontecendo.

\section{Perspectivas}

Nosso próximo passo é fazer um survey com as 100 fontes extragalácticas mais brilhantes do 3FGL (ou, possivelmente, do 4FGL, que está em produção com base nos seis primeiros anos de operações do Fermi) na busca por flares e periodicidade. Como o processo será de alto custo computacional, sua realização só será praticável com a utilização do Alphacrucis ou outro cluster. O critério de seleção destas fontes é simplesmente sua alta significância estatística, o que facilitará a convergência da likelihood analysis.

Continuaremos monitorando o caráter periódico das fontes PG1553+113, na tentativa de comprovar que sua periodicidade cresce linearmente com o tempo, e NGC 1275, cuja periodicidade e interpretação física ainda não são muito claras. As três fontes recém descobertas também continuarão sendo monitoradas em raios $\gamma$, e procuraremos por suas componentes em outros comprimentos de onda num momento oportuno. 
Este trabalho nos trouxe algumas conclusões e abriu um leque de novas questões que serão perseguidas durante o projeto de doutorado. Os resultados obtidos serão reportados em um artigo que será submetido ao ApJ ou MNRAS. Muito do que foi feito continuará sendo aproveitado e desenvolvido, para que possamos dar continuidade da melhor maneira possível ao nosso estudo sobre a variabilidade de AGNs em raios gama no Universo local. 


\section{Referências Bibliográficas}

Abdo, A., Ackermann, M., Ajello, M., Allafort, A., Antolini, E., Atwood, W., Axelsson, M., Baldini, L., Ballet, J., Barbiellini, G., et al. (2010a). Fermi large area telescope first source catalog. The Astrophysical Journal Supplement Series, 188(2):405.

Abdo, A. A., Ackermann, M., Ajello, M., Allafort, A., Antolini, E., Atwood, W., Axelsson, M., Baldini, L., Ballet, J., Barbiellini, G., et al. (2010b). The first catalog of active galactic nuclei detected by the fermi large area telescope. The Astrophysical Journal, $715(1): 429$.

Acero, F., Ackermann, M., Ajello, M., Albert, A., Atwood, W., Axelsson, M., Baldini, L., Ballet, J., Barbiellini, G., Bastieri, D., et al. (2015a). Fermi large area telescope third source catalog. The Astrophysical Journal Supplement Series, 218(2):23.

Acero, F., Ackermann, M., Ajello, M., Albert, A., Atwood, W., Axelsson, M., Baldini, L., Ballet, J., Barbiellini, G., Bastieri, D., et al. (2015b). Fermi large area telescope third source catalog. The Astrophysical Journal Supplement Series, 218(2):23.

Ackermann, M., Ajello, M., Albert, A., Atwood, W., Baldini, L., Ballet, J., Barbiellini, G., Bastieri, D., Bechtol, K., Bellazzini, R., et al. (2015a). The spectrum of isotropic diffuse gamma-ray emission between $100 \mathrm{mev}$ and 820 gev. The Astrophysical Journal, 799(1):86.

Ackermann, M., Ajello, M., Albert, A., Atwood, W., Baldini, L., Ballet, J., Barbiellini, G., Bastieri, D., Gonzalez, J. B., Bellazzini, R., et al. (2015b). Multiwavelength evidence for quasi-periodic modulation in the gamma-ray blazar pg $1553+113$. The Astrophysical Journal Letters, 813(2):L41.

Ackermann, M., Ajello, M., Albert, A., Atwood, W., Baldini, L., Barbiellini, G., Bastieri, D., Bechtol, K., Bellazzini, R., Bissaldi, E., et al. (2015c). Search for extended gamma- 
ray emission from the virgo galaxy cluster with fermi-lat. The Astrophysical Journal, $812(2): 159$.

Ackermann, M., Ajello, M., Atwood, W., Baldini, L., Ballet, J., Barbiellini, G., Bastieri, D., Gonzalez, J. B., Bellazzini, R., Bissaldi, E., et al. (2016). 2fhl: The second catalog of hard fermi-lat sources. The Astrophysical Journal Supplement Series, 222(1):5.

Actis, M., Agnetta, G., Aharonian, F., Akhperjanian, A., et al. (2010). Design concepts for the cherenkov telescope array. arXiv preprint arXiv:1008.3703.

Ajello, M., Gasparrini, D., Sánchez-Conde, M., Zaharijas, G., Gustafsson, M., CohenTanugi, J., Dermer, C., Inoue, Y., Hartmann, D., Ackermann, M., et al. (2015a). The origin of the extragalactic gamma-ray background and implications for dark matter annihilation. The Astrophysical Journal Letters, 800(2):L27.

Ajello, M., Gasparrini, D., Sánchez-Conde, M., Zaharijas, G., Gustafsson, M., CohenTanugi, J., Dermer, C., Inoue, Y., Hartmann, D., Ackermann, M., et al. (2015b). The origin of the extragalactic gamma-ray background and implications for dark matter annihilation. The Astrophysical Journal Letters, 800(2):L27.

Atwood, W., Abdo, A. A., Ackermann, M., Althouse, W., Anderson, B., Axelsson, M., Baldini, L., Ballet, J., Band, D., Barbiellini, G., et al. (2009). The large area telescope on the fermi gamma-ray space telescope mission. The Astrophysical Journal, 697(2):1071.

Bloomfield, P. (2004). Fourier analysis of time series: an introduction. John Wiley \& Sons.

Bohm, G. and Zech, G. (2010). Introduction to statistics and data analysis for physicists. DESY.

Böttcher, M., Basu, S., Joshi, M., Villata, M., Arai, A., Aryan, N., Asfandiyarov, I., Bach, U., Bachev, R., Berduygin, A., et al. (2007). The webt campaign on the blazar 3c 279 in 2006for questions regarding the availability of the data from the webt campaign presented in this paper, please contact the webt president massimo villata at. The Astrophysical Journal, 670(2):968.

Bridle, A. H., Hough, D. H., Lonsdale, C. J., Burns, J. O., and Laing, R. A. (1994). Deep vla imaging of twelve extended 3cr quasars. The Astronomical Journal, 108:766-820.

Caproni, A. and Abraham, Z. (2004). Precession in the inner jet of 3c 345. The Astrophysical Journal, 602(2):625. 
Domínguez, A., Finke, J., Prada, F., Primack, J., Kitaura, F., Siana, B., and Paneque, D. (2013). Detection of the cosmic $\gamma$-ray horizon from multiwavelength observations of blazars. The Astrophysical Journal, 770(1):77.

Dong, X. and De Robertis, M. M. (2006). Low-luminosity active galaxies and their central black holes. The Astronomical Journal, 131(3):1236.

Dunn, R., Fabian, A., and Sanders, J. (2006). Precession of the super-massive black hole in ngc 1275 (3c 84)? Monthly Notices of the Royal Astronomical Society, 366(3):758-766.

Fabian, A., Johnstone, R., Sanders, J., Conselice, C., Crawford, C., Gallagher III, J., and Zweibel, E. (2008). Magnetic support of the optical emission line filaments in ngc 1275. Nature, 454(7207):968-970.

Fabian, A., Sanders, J., Allen, S., Canning, R., Churazov, E., Crawford, C., Forman, W., GaBany, J., Hlavacek-Larrondo, J., Johnstone, R., et al. (2011). A wide chandra view of the core of the perseus cluster. Monthly Notices of the Royal Astronomical Society, 418(4):2154-2164.

Fabian, A., Sanders, J. S., Taylor, G., Allen, S., Crawford, C., Johnstone, R., and Iwasawa, K. (2006). A very deep chandra observation of the perseus cluster: shocks, ripples and conduction. Monthly Notices of the Royal Astronomical Society, 366(2):417-428.

Falceta-Gonçalves, D., Caproni, A., Abraham, Z., Teixeira, D., and Dal Pino, E. d. G. (2010). Precessing jets and x-ray bubbles from ngc 1275 (3c 84) in the perseus galaxy cluster: A view from three-dimensional numerical simulations. The Astrophysical Journal Letters, 713(1):L74.

Fanaroff, B. and Riley, J. (1974). The morphology of extragalactic radio sources of high and low luminosity. Monthly Notices of the Royal Astronomical Society, 167(1):31P$36 \mathrm{P}$.

Ferrarese, L., Ford, H. C., and Jaffe, W. (1996). Evidence for a massive black hole in the active galaxy ngc 4261 from hubble space telescope images and spectra. The Astrophysical Journal, 470:444.

Finke, J. D., Razzaque, S., and Dermer, C. D. (2010). Modeling the extragalactic background light from stars and dust. The Astrophysical Journal, 712(1):238.

Gebhardt, K., Adams, J., Richstone, D., Lauer, T. R., Faber, S., Gültekin, K., Murphy, J., and Tremaine, S. (2011). The black hole mass in $\mathrm{m} 87$ from gemini/nifs adaptive optics observations. The Astrophysical Journal, 729(2):119. 
Gillessen, S., Genzel, R., Fritz, T., Quataert, E., Alig, C., Burkert, A., Cuadra, J., Eisenhauer, F., Pfuhl, O., Dodds-Eden, K., et al. (2012). A gas cloud on its way towards the supermassive black hole at the galactic centre. Nature, 481(7379):51-54.

Gilmore, R. C., Somerville, R. S., Primack, J. R., and Domínguez, A. (2012). Semianalytic modelling of the extragalactic background light and consequences for extragalactic gamma-ray spectra. Monthly Notices of the Royal Astronomical Society, 422(4):3189-3207.

Giommi, P., Padovani, P., Polenta, G., Turriziani, S., D'Elia, V., and Piranomonte, S. (2012). A simplified view of blazars: clearing the fog around long-standing selection effects. Monthly Notices of the Royal Astronomical Society, 420(4):2899-2911.

Groves, B. (2006). The narrow line region: Current models and future questions. arXiv preprint astro-ph/0612309.

Ho, L. C. (2009). Radiatively inefficient accretion in nearby galaxies. The Astrophysical Journal, 699(1):626.

Ho, L. C., Filippenko, A. V., and Sargent, W. L. (1997a). A search for dwarf"seyfert nuclei. iii. spectroscopic parameters and properties of the host galaxies. The Astrophysical Journal Supplement Series, 112(2):315.

Ho, L. C., Filippenko, A. V., and Sargent, W. L. (1997b). A search for dwarf"seyfert nuclei. v. demographics of nuclear activity in nearby galaxies. The Astrophysical Journal, $487(2): 568$.

Konopelko, A., Mastichiadis, A., Kirk, J., de Jager, O. C., and Stecker, F. W. (2003). Modeling the tev gamma-ray spectra of two low-redshift active galactic nuclei: Markarian 501 and markarian 421. The Astrophysical Journal, 597(2):851.

Kormendy, J., Drory, N., Bender, R., and Cornell, M. E. (2010). Bulgeless giant galaxies challenge our picture of galaxy formation by hierarchical clusteringbased on observations obtained with the hobby-eberly telescope, which is a joint project of the university of texas at austin, the pennsylvania state university, stanford university, ludwig-maximilians-universität münchen, and georg-august-universität göttingen., based on observations made with the nasa/esa hubble space telescope, obtained from the data archive at stsci, which is operated by aura, inc., under nasa contract nas 5-26555. these observations are associated with program numbers 7330, 7919, 8591, 8597, 8599, 9293, 9360, 9490, 9788, and 11080. The Astrophysical Journal, 723(1):54.

Laing, R. A. and Bridle, A. H. (1987). Rotation measure variation across m84. Monthly Notices of the Royal Astronomical Society, 228(3):557-571. 
Liu, P. C. (1999). A fifteen minutes introduction of wavelet transform and applications. In Proceedings, ASCE 1999 International Water Resources Engineering Conference.

Lobanov, A. (2004). Mergers and binary systems of smbh in the contexts of nuclear activity and galaxy evolution. In Growing Black Holes: Accretion in a Cosmological Context, pages 354-355. Springer.

Mahadevan, R., Narayan, R., and Krolik, J. (1997). Gamma-ray emission from advectiondominated accretion flows around black holes: application to the galactic center. The Astrophysical Journal, 486(1):268.

Massaro, F., Thompson, D. J., and Ferrara, E. C. (2016). The extragalactic gamma-ray sky in the fermi era. The Astronomy and Astrophysics Review, 24(1):1-58.

Merloni, A., Heinz, S., and Di Matteo, T. (2003). A fundamental plane of black hole activity. Monthly Notices of the Royal Astronomical Society, 345(4):1057-1076.

Mezcua, M. and Prieto, M. (2014). Evidence of parsec-scale jets in low-luminosity active galactic nuclei. The Astrophysical Journal, 787(1):62.

Miller, J., Goodrich, R., and Mathews, W. G. (1991). Multidirectional views of the active nucleus of ngc 1068. The Astrophysical Journal, 378:47-64.

Morgan, W. (1968). A comparison of the optical forms of certain seyfert galaxies with the n-type radio galaxies. The Astrophysical Journal, 153:27.

Nemmen, R. S., Storchi-Bergmann, T., and Eracleous, M. (2014). Spectral models for low-luminosity active galactic nuclei in liners: the role of advection-dominated accretion and jets. Monthly Notices of the Royal Astronomical Society, page stt2388.

Nemmen, R. S. and Tchekhovskoy, A. (2015). On the efficiency of jet production in radio galaxies. Monthly Notices of the Royal Astronomical Society, 449(1):316-327.

Netzer, H. (2013). The Physics and Evolution of Active Galactic Nuclei. Cambridge University Press.

Osterbrock, D. E. (1984). Active galactic nuclei. Quarterly Journal of the Royal Astronomical Society, 25:1.

Padovani, P., Giommi, P., Polenta, G., Turriziani, S., D'Elia, V., and Piranomonte, S. (2012). A simplified view of blazars: why bl lacertae is actually a quasar in disguise*. arXiv preprint arXiv:1205.064\%.

Paliya, V. S. (2015). Fermi-large area telescope observations of the exceptional gamma-ray flare from 3c 279 in 2015 june. The Astrophysical Journal Letters, 808(2):L48. 
Rudnick, D. L. and Davis, R. E. (2003). Red noise and regime shifts. Deep Sea Research Part I: Oceanographic Research Papers, 50(6):691-699.

Rybicki, G. B. and Lightman, A. P. (2008). Radiative processes in astrophysics. John Wiley \& Sons.

Scargle, J. D. (1982). Studies in astronomical time series analysis. ii-statistical aspects of spectral analysis of unevenly spaced data. The Astrophysical Journal, 263:835-853.

Schneider, P. (2014). Extragalactic astronomy and cosmology: an introduction. Springer.

Seyfert, C. K. (1943). Nuclear emission in spiral nebulae. The Astrophysical Journal, $97: 28$.

Shatkay, H. (1995). The fourier transform-a primer. Brown University.

Shu, F. (1991). The physics of astrophysics, vol. 1: Radiation. univ.

Tchekhovskoy, A., Narayan, R., and McKinney, J. C. (2011). Efficient generation of jets from magnetically arrested accretion on a rapidly spinning black hole. Monthly Notices of the Royal Astronomical Society: Letters, 418(1):L79-L83.

Thompson, D. J., Digel, S. W., and Racusin, J. L. (2012). Exploring the extreme universe with the fermi gamma-ray space telescope.

Torrence, C. and Compo, G. P. (1998). A practical guide to wavelet analysis. Bulletin of the American Meteorological society, 79(1):61-78.

Wilman, R., Edge, A., and Johnstone, R. (2005). The nature of the molecular gas system in the core of ngc 1275. Monthly Notices of the Royal Astronomical Society, 359(2):755764.

Wood, M. (2016). Fermi lat performance. https://www.slac.stanford.edu/exp/ glast/groups/canda/lat_Performance.htm. [Online; accessed 08-Janeiro-2016]. 